\title{
WestVirginiaUniversity
}

THE RESEARCH REPOSITORY @ WVU

Graduate Theses, Dissertations, and Problem Reports

2012

\section{Microfluidic Based Human Blood Plasma Separation}

\author{
Ruizhi Zhong \\ West Virginia University
}

Follow this and additional works at: https://researchrepository.wvu.edu/etd

\section{Recommended Citation}

Zhong, Ruizhi, "Microfluidic Based Human Blood Plasma Separation" (2012). Graduate Theses, Dissertations, and Problem Reports. 3349.

https://researchrepository.wvu.edu/etd/3349

This Thesis is protected by copyright and/or related rights. It has been brought to you by the The Research Repository @ WVU with permission from the rights-holder(s). You are free to use this Thesis in any way that is permitted by the copyright and related rights legislation that applies to your use. For other uses you must obtain permission from the rights-holder(s) directly, unless additional rights are indicated by a Creative Commons license in the record and/ or on the work itself. This Thesis has been accepted for inclusion in WVU Graduate Theses, Dissertations, and Problem Reports collection by an authorized administrator of The Research Repository @ WVU. For more information, please contact researchrepository@mail.wvu.edu. 


\title{
Microfluidic Based Human Blood Plasma Separation
}

\author{
Ruizhi Zhong
}

Thesis Submitted to the

Benjamin M. Statler College of Engineering and Mineral Resources at West Virginia University

in partial fulfillment of the requirements for the degree of

\author{
Master of Science \\ in \\ Mechanical Engineering \\ Yuxin Liu, Ph.D., Advisor and Chair \\ Nianqiang Wu, Ph.D., Co-Advisor \\ Ismail Celik, Ph.D. \\ Department of Mechanical and Aerospace Engineering \\ Morgantown, West Virginia \\ 2012
}

Keywords: Microfluidics; human blood; active separation; passive separation 


\title{
ABSTRACT \\ Microfluidic Based Human Blood Plasma Separation
}

\begin{abstract}
Ruizhi Zhong
Microfluidic based biotechnologies have been widely applied for biological and biomedical research and applications because the physical scales of microfluidics are suitable for biological cells and provide the ability and flexibility to control spatiotemporal biochemical parameters. The current efforts for biosensor technologies are aiming to develop detection technologies directly from small volumes of the human fluids, such as blood, urea, and saliva, for accurate, fast, and affordable detection and analysis.
\end{abstract}

Human blood provides information and supports the functioning of human body, such as nourishing tissues, regulating organs activities and defending harmful material. Blood components are the prime interests for biosensor applications in medicine research. Separation of different blood components, such as plasma, red blood cells, or white blood cells, is important for specific downstream detections (such as cancer diagnosis and biohazard detections) by preventing contamination of the plasma from blood cells' DNA. Conventional methods for blood separation include flow cytometry, centrifugation and filtration. However, high levels of skills, large volumes of samples and long processing time are usually required in these methods. Microfluidic methods for blood separation can provide advantages over these conventional methods, such as small blood volume handling, easy fabrication, fast processing, and miniaturized and portable devices for point-of-care analysis.

In this thesis work, we investigated two different microfluidic approaches for human blood separation: 1) Hydrodynamic bifurcation law based continuous blood separation. The bifurcation law, centrifugation force and Fåhræus-Lindqvist effect were employed in the approach. By using the COMSOL Multiphysics simulation, we optimized the device design, and the efficiency of blood separation of the microfluidic device was conducted using samples with different hematocrit levels and running velocities. A series of experiments were performed to investigate and quantify the two effects. The experimental results showed that the multiple bifurcation devices can provide plasma separation with high separation efficiency and yield. Electrophoresis analysis was used to examine major protein components in the separated plasma from microfluidic devices compared with that separated by a conventional centrifuge method and showed that the major proteins presented in both testing groups. 2) Membrane based passive blood separation. In this approach, a microfluidic capillary pump and a commercial Vivid ${ }^{\mathrm{TM}}$ plasma separation membrane were integrated to separate, collect and deliver the separated plasma from a finger-prick whole blood. 
The plasma released from the membrane was collected and drawn into the microfluidic capillary pump for further downstream detection. It was demonstrated that the membrane can separate plasma depending on the natural forces of capillary action without any active pumping or electricity to operate.

Our microfluidic plasma separators are expected to be integrated with other sensing elements into a reliable, cost-effective and field-deployable biosensor for detection of heavy metals and biomarkers from a small volume of human blood. In this regard, an integration lab-on-a-chip microfluidic system needs to be further investigated, in which on-chip valves and fluid controls will be used for the system integration. 


\section{ACKNOWLEDGEMENTS}

I would like to express my gratitude to my research advisor Dr. Yuxin Liu for providing me the opportunity to do research under her guidance. I am sincerely grateful to my committee members for their support and guidance on my research. Special thanks to all members in Dr. Liu's group for their help in my research. Thanks to WVU Shared Research Facilities (Cleanroom facilities) and Microfluidic Integrative Cellular Research on Chip Laboratory (MICRoChip Lab) at WVU. The research work is supported by National Science Foundation grant EPS-1003907. 


\section{TABLE OF CONTENTS}

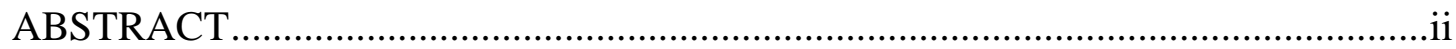

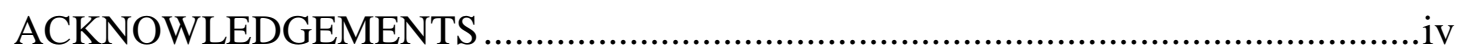

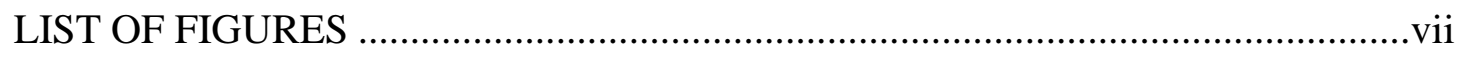

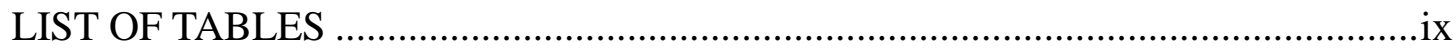

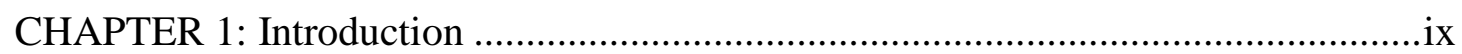

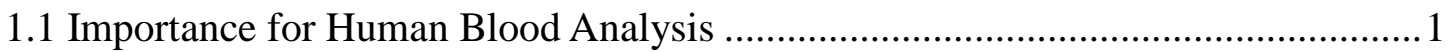

1.2 Research and Clinical Needs for Blood Plasma Separation .................................2

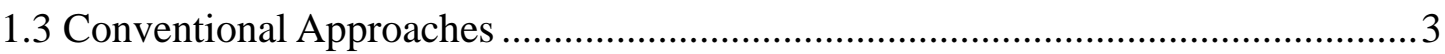

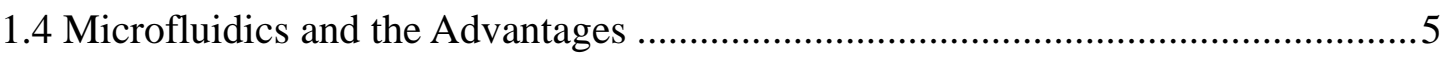

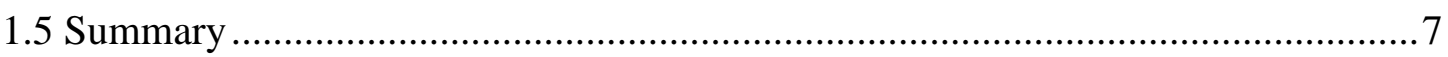

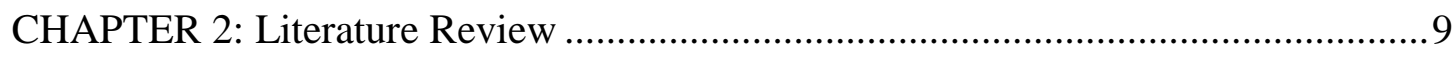

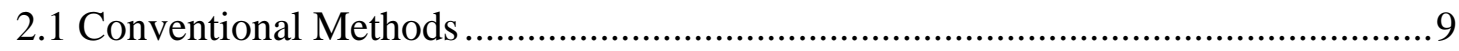

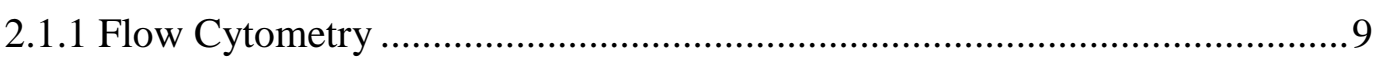

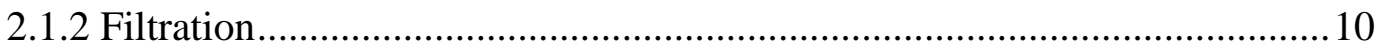

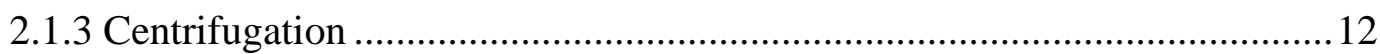

2.2 Micro-scale Technologies for Blood Processing .................................................. 14

2.2.1 Mechanical Force Based Blood Separation ............................................... 14

2.2.2 Dielectrophoresis Based Blood Separation.................................................16

2.2.3 Optical Interaction Based Blood Separation............................................. 17

2.2.4 Magnetic Based Blood Separation ......................................................... 18

2.2.3 Biomedical Interaction Based Blood Separation ........................................ 19

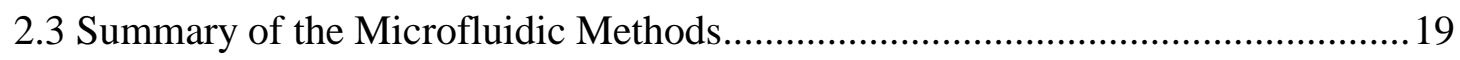

CHAPTER 3: Principles for Microfluidic Based Plasma Separation ..........................23

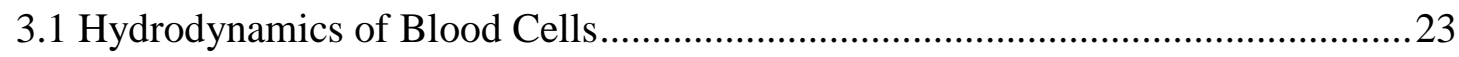

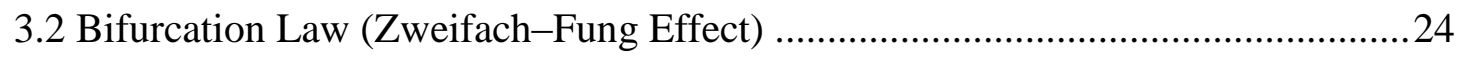

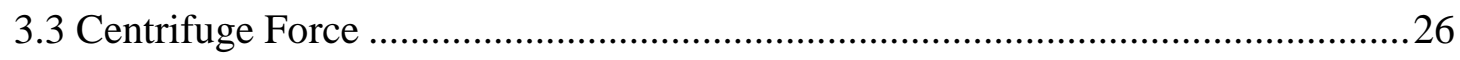

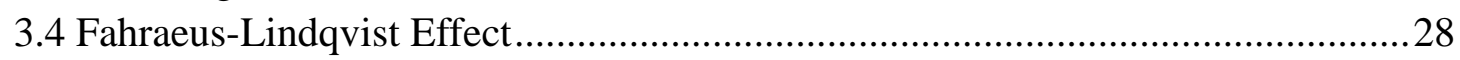

CHAPTER 4: Microfluidic Device Design and Fabrication ...................................29

4.1 Single Bifurcation Microfluidic Device..............................................................29

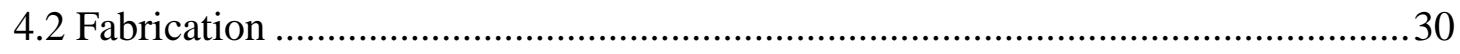

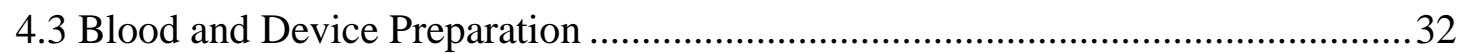

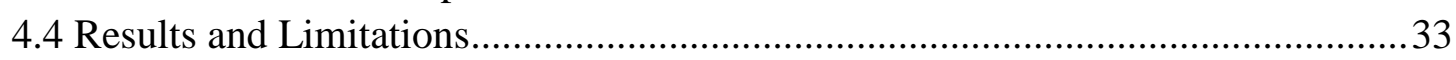

Chapter 5: Microfluidic Multiple Bifurcation Device and COMSOL Simulation .......36

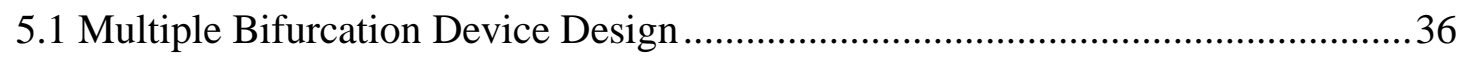

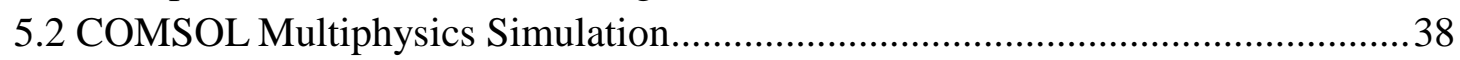

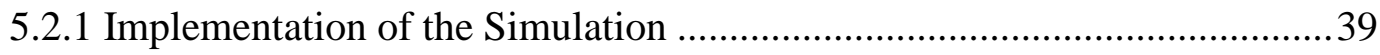

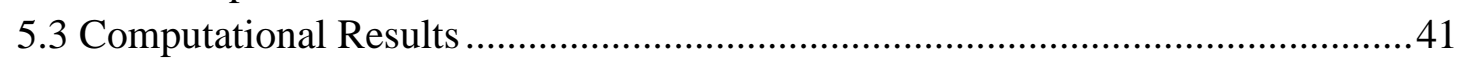

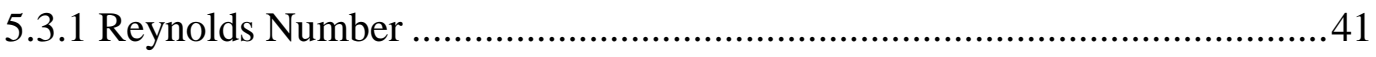

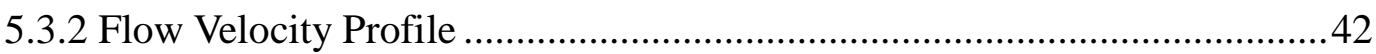

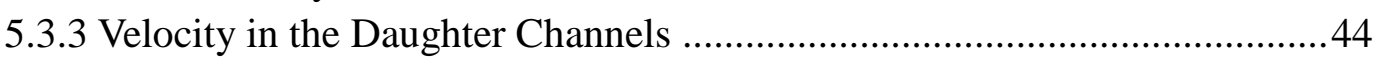


5.3.4 Flow Rate Ratios . .44

CHAPTER 6: Experimental Results of Multiple Bifurcation Device ..........................48

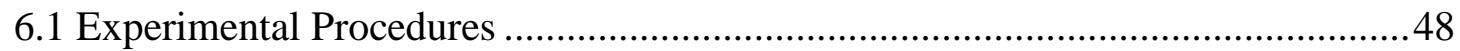

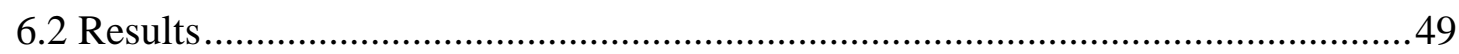

6.3 Hydrodynamic Effects on Blood Separation ...................................................51

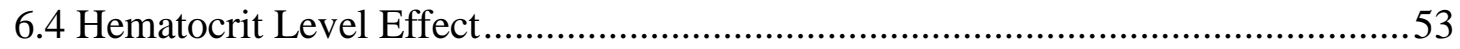

6.5 Running Speed Effect ........................................................................................5

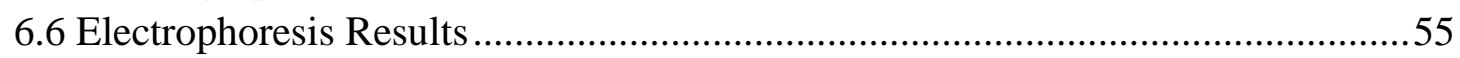

CHAPTER 7: Microfluidic Membrane Based Plasma Separation ..............................58

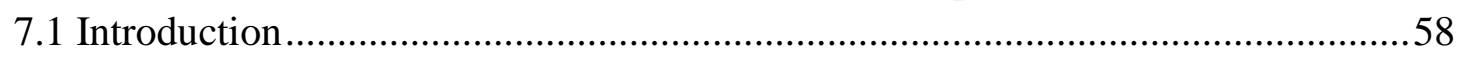

7.2 Vivid Plasma Separation Membrane ....................................................................61

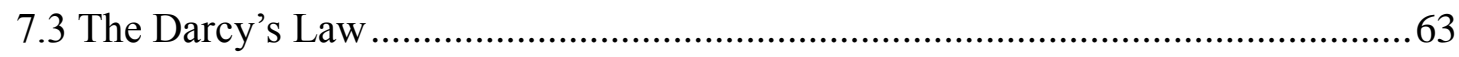

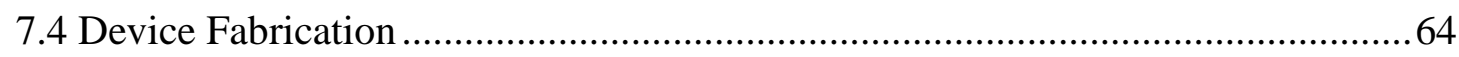

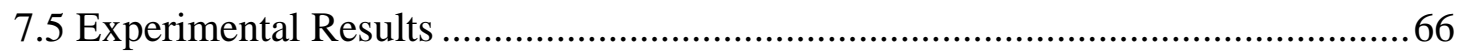

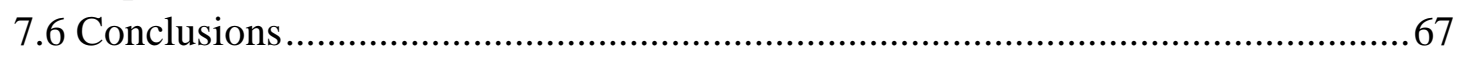

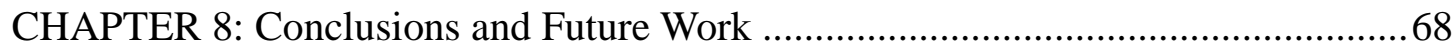

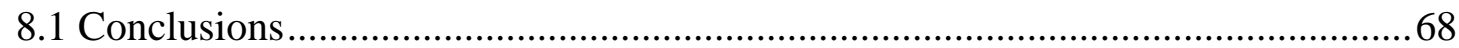

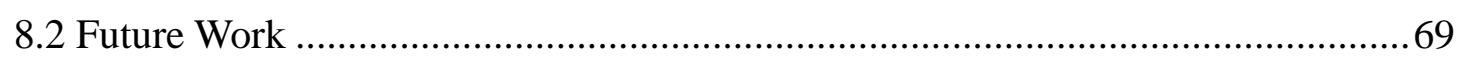

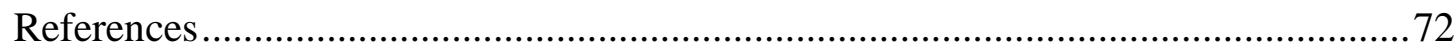




\section{LIST OF FIGURES}

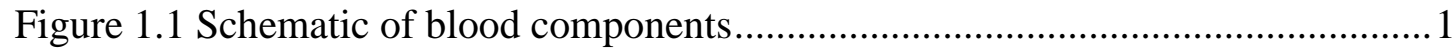

Figure 1.2 Illustration of heavy metal hazards to human body....................................2 Figure 1.3 (a) Flow cytometer. (b) Centrifuge. (c) Continuous renal replacement

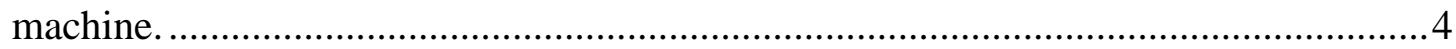

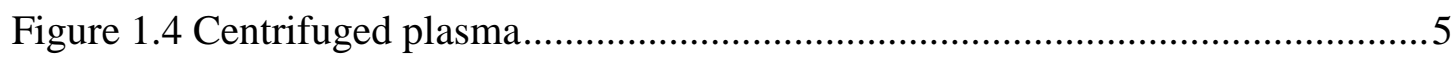

Figure1.5 A microfluidic biosensor device for cancer detection ................................6

Figure 2.1 Schematic diagram of the flow cytometer ............................................... 10

Figure 2.2 (a) The side view and the top view of a microfluidic filtration system. (b)

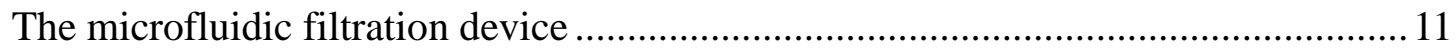

Figure 2.3 A microfluidic centrifuge device .............................................................. 13

Figure 2.4 (a) Offset array of simple microposts set across a 500-mm-wide silicon channel. (b) Array of complex microposts separated by 7-mm-wide tortuous channels.

Figure 2.5 Obstacle array designs for trapping cells................................................. 15

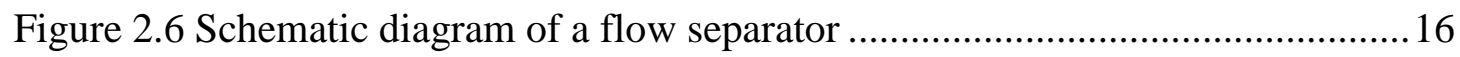

Figure 2.7 Schematic design of an integrated microfluidic sorting system .................17

Figure 2.8 (a) Schematic diagram of a continuous magnetic separator having one inlet and three outlets. (b) Cross-section view of the separator in the paramagnetic capture

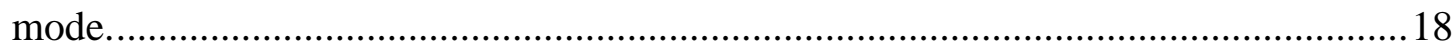

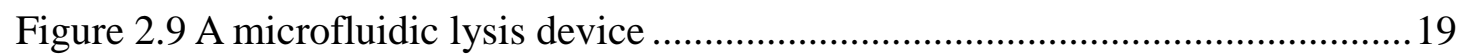

Figure 3.1 (a) Schematic diagram for interpreting the bifurcation law. (b) An

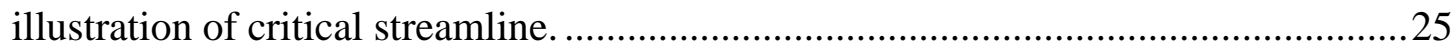

Figure 3.2 Schematic diagram for an example with a 100\% separation......................26

Figure 3.3 Microfluidic bend channel structure for assisting blood separation...........27

Figure 4.1 The schematic design for microfluidic single bifurcation device ..............29

Figure 4.2 Schematic process for microfluidic device fabrication ............................. 31

Figure 4.3 The picture shows a syringe loaded with blood hooked on a syringe pump .33

Figure 4.4 Results of single bifurcation device A) Design 1 for the microfluidic device. The frame represents the blood plasma separation region in B, C, and D. B)-D) show the blood separation at different time as indicated in the figures. The blood flow rate was kept at $3 \mu \mathrm{l} / \mathrm{min}$ and the red dashed curve indicated the path of blood cells in the

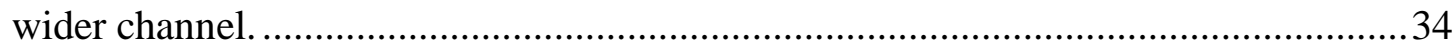

Figure 5.1 The schematic design for microfluidic multiple bifurcation device............36

Figure 5.2 Reynolds numbers in the main channel and the daughter channels ...........42 Figure 5.3 (a) Velocity filed of the multiple bifurcation device; (b) A detail view at the

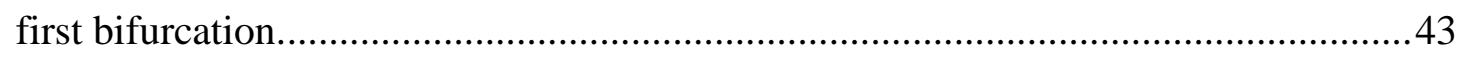

Figure 5.4 Flow velocities of the daughter channels ...............................................4

Figure 5.5 The critical streamline of (a) Cell-to-vessel diameter ratio is of the order of one; and (b) Cell-to-vessel diameter ratio is over the order of one. 46 
Figure 6.1 (a) The schematic of experimental setup. (b) Experimental setup for blood separation with real-time monitoring.

Figure 6.2 (a) Plasma separation at 1st and 2nd bifurcation. (b) Plasma separation at 3rd and 4th bifurcation. (c) Plasma separation at 5th and 6th bifurcation.

Figure 6.3 A) A microfluidic device. B) Blood separation in the microfluidic device. C) Higher magnification showing blood separation in the device.................................52 Figure 6.4 (a) Hematocrit level effects on the separation efficiency. (b) Hematocrit level effects on the plasma separation yield..........................................................53 Figure 6.5 Separation efficiency at different running speeds when the hematocrit level

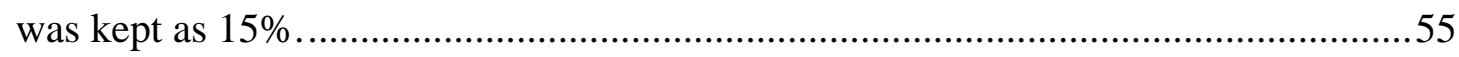

Figure 6.6 Microfluidic separated blood plasma (hematocrit level: 15\%) .................56 Figure 6.7 Electrophoresis analysis of (a) centrifuged plasma and (b) microfluidic

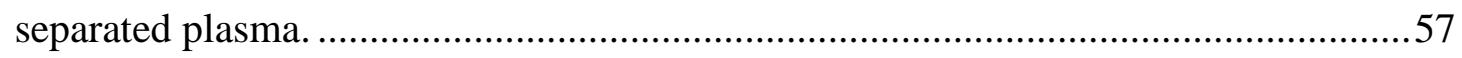

Figure 7.1 Clamping of the membrane between two PDMS layers ..........................59 Figure 7.2 (a) Schematic design of crossed microfluidic channels separated by a nanometer-diameter capillary array interconnect. (b) Schematic design of a parallel channel system. . .60

Figure 7.3 Photograph of a microchip with crossed microchannels and a nanofluidic

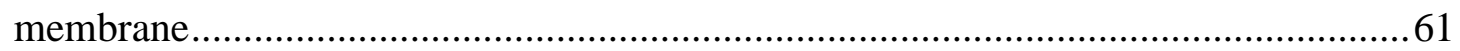

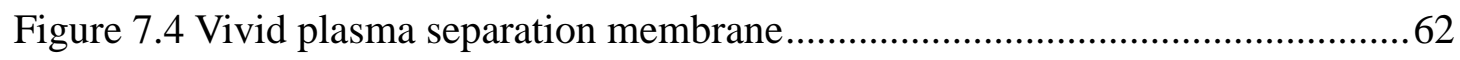

Figure 7.5 High quality plasma generation using vivid plasma membrane.................63

Figure 7.6 Working mechanism of Darcy's law in two dimensions ...........................63

Figure 7.7 Schematic fabrication process of the microfluidic membrane based device

Figure 7.8 Microfluidic device pattern of membrane based blood plasma separation 65

Figure 7.9 The membrane based device.............................................................66

Figure 7.10 The picture shows the plasma separation using a membrane based device

Figure 8.1 Lab on a Chip

Figure 8.2 (a) Photograph of an array of complementary organic inverters on $1 \mathrm{~mm}$ thick transparent PDMS. (b) The detailed view of inverter device. (c) Schematic design of the complementary inverter stack directly prepared on PDMS. .70 Figure 8.3 An integrated microfluidic electrochemical sensor based on planar electrodes. 


\section{LIST OF TABLES}

Table 3.1 Illustration of the different regimes of cell behavior in a microchannel with dimensions below $300 \mu \mathrm{m}$ .23

Table 5.1 Flow rate ratios at bifurcations for Newtonian and Non-Newtonian fluid..39

Table 5.2 Flow rate ratio chart..... .45 


\section{CHAPTER 1: Introduction}

\subsection{Importance for Human Blood Analysis}

Human blood provides information and supports the functioning of human body, such as nourishing tissues, regulating organs activities and defending harmful materials. As shown in Figure 1.1, blood has two main components: plasma and cells, which accounts for $55 \%$ and $45 \%$ of the volume of the blood, respectively. The blood cells include red blood cells, white blood cells and platelets, and these cells and blood components are transported through blood circulation system ${ }^{1}$.

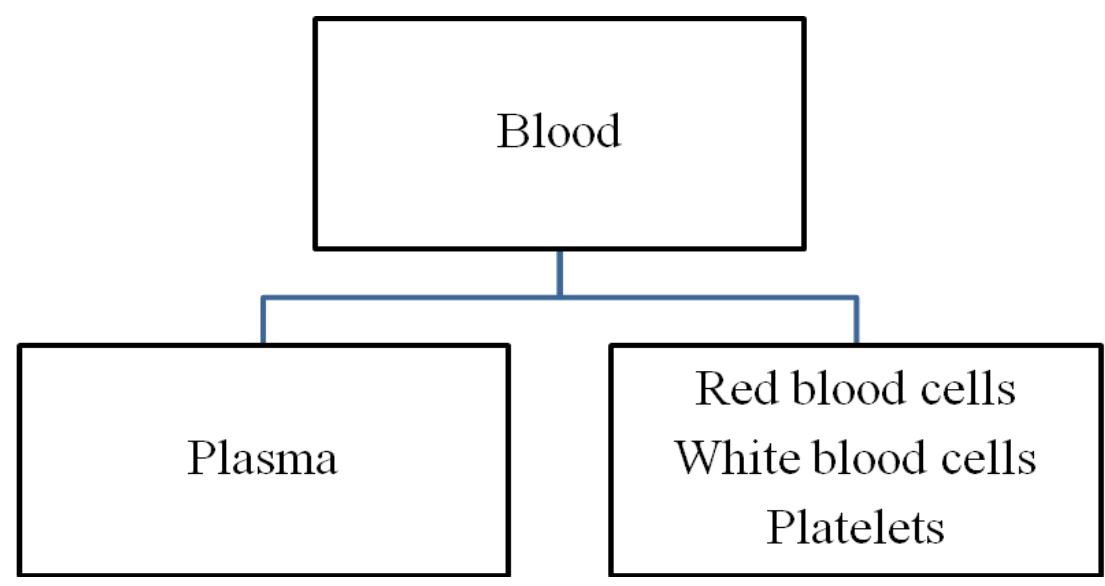

Figure 1.1 Schematic of blood components

The inorganic salts, oxygen, hormones, enzymes, antibodies and the product of cell metabolism in the blood can provide a lot of physiological functions. As a result, blood harbors a massive amount of information about the functioning of tissues and organs in the body ${ }^{2}$, and the analysis of blood components have been adopted as a useful approach for medical diagnosis, such as cancer biomarkers detection for earlier cancer detection and heavy metal detection for biohazard analysis. Hence the research of analyzing blood cells or blood components is of great interests and importance for 
biomedical research.

\subsection{Research and Clinical Needs for Blood Plasma Separation}

Blood components are the prime interests for biosensor applications in medicine research. Separation of different blood components, such as plasma, red blood cells, or white blood cells, is important for specific downstream detections, such as by preventing contamination of the plasma from blood cells' DNA. Thus, the extraction of plasma from whole blood is the first step in many biological assay protocols and critical important in medical diagnostics.

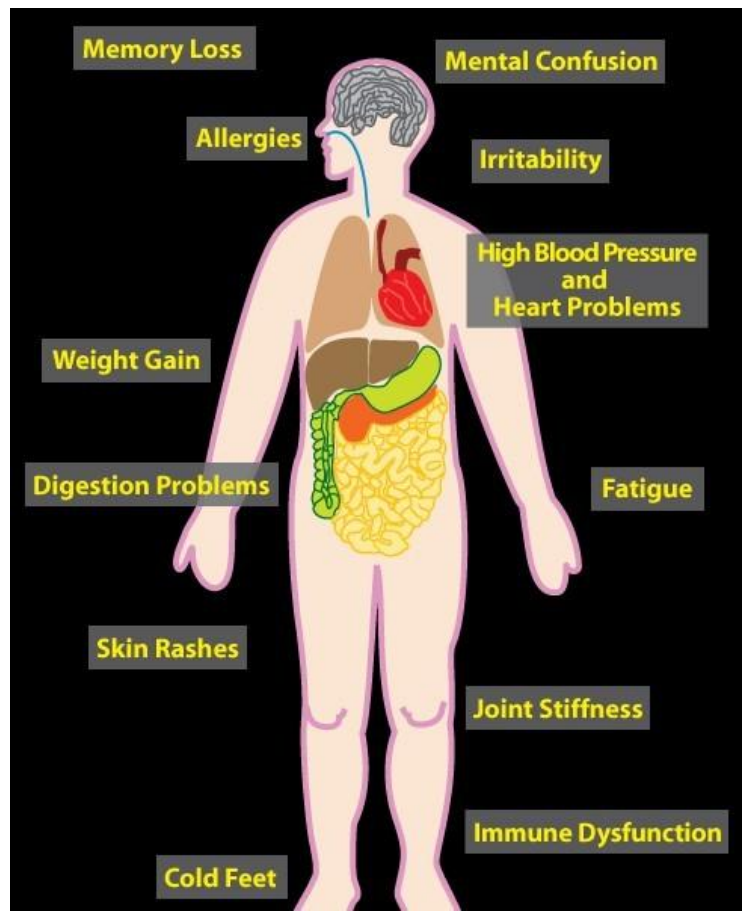

Figure 1.2 Illustration of heavy metal hazards to human body ${ }^{3}$

Nowadays, environmental and occupational exposure to heavy metals is an emerging health concern because heavy metals can cause a lot of diseases, such as memory loss, mental confusion, fatigue, high blood pressure and heart problems, and immune dysfunction as shown in Figure 1.2. In Kaloyiannia's paper, they investigated toxic 
compounds, such as heavy metals (zinc and cadmium) and organic pollutants in mussel haemolymph, and provided valuable information for environmental monitoring ${ }^{4}$.

In order to take further measurements to treat diseases caused by heavy metals, it is important to firstly detect heavy metals and determine their concentrations from real world samples. In human blood, the heavy metals exist as ions and conjugated with proteins in blood plasma, and the existence of blood cells can influence the results of detection. Because of these, in most cases, the initial step for heavy metal detection is to get purified plasma by eliminating or reducing the influence of cells by the separation of plasma from other blood cells.

\subsection{Conventional Approaches}

Conventional methods for cell identification and plasma separation include flow cytometer, centrifugation and filtration. Figure 1.3 shows the pictures of those conventional machines for blood plasma separation, which are flow cytometer, centrifuge and renal replacement machine, respectively. For flow cytometer, detailed information of working mechanism will be discussed in the next chapter.

Figure 1.3 (b) shows a centrifuge machine. Typically, the plasma separation is performed at $5000 \mathrm{rpm}$ for 10 minutes and the separated plasma is shown as in Figure 1.4, in which the upper fluid layer is the separated plasma and the dark red layer at the bottom is the cells layer. Figure 1.3 (c) shows a renal replacement machine. This 
machine is based on filtration method and used in medical or clinical lab for obtaining larger volumes of plasma.
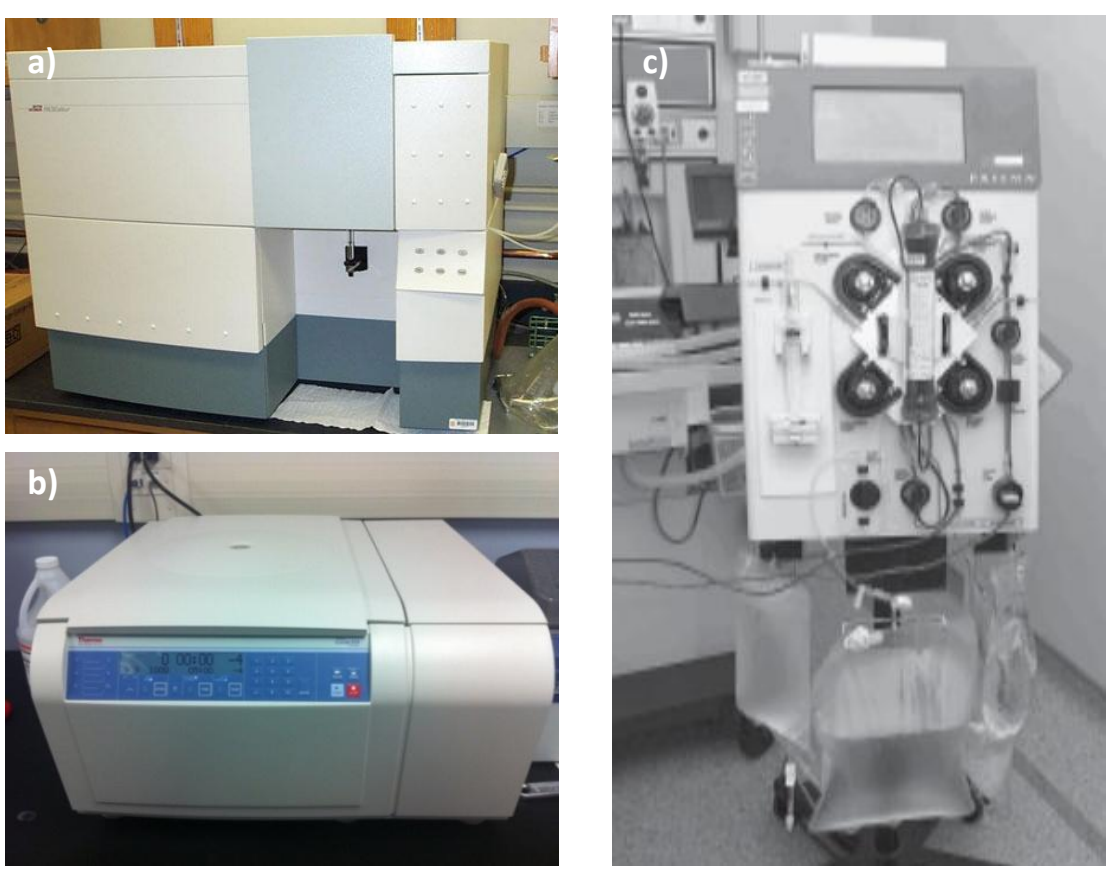

Figure 1.3 (a) Flow cytometer. (b) Centrifuge. (c) Continuous renal replacement machine.

Although the conventional methods are very powerful for plasma separation and most commonly used in research and clinical labs, especially for processing larger volumes of blood. However, those methods have many limitations for needs requiring fast processing and small sample volumes. In addition, the machine operations and result analysis need high levels of skills. For example, the flow cytometer is expensive and hard for maintenance. For centrifugation, cell damage is the main concern because hemolysis occurs during high speed centrifugation ${ }^{5}$. For filtration, the clog is a problem which always presents. Additionally, all these methods require external powers and are not suitable for personal use. All of these are difficultly making some medical detections in developing counties or rural areas. 


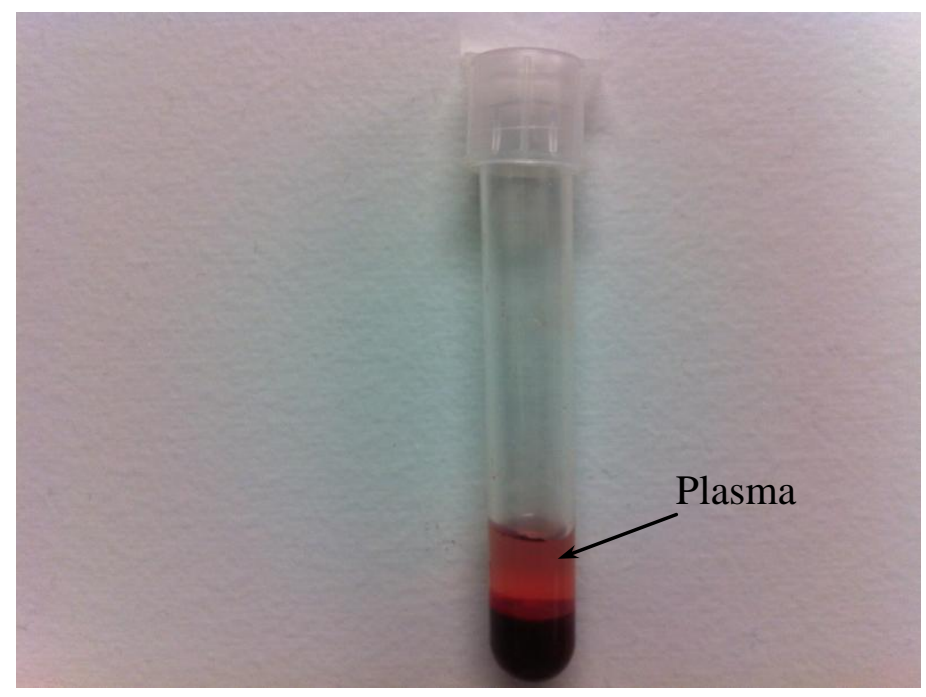

Figure 1.4 Centrifuged plasma

\subsection{Microfluidics and the Advantages}

Microfluidic based biotechnologies have been widely applied for biological and biomedical research and applications because the physical scales of microfluidics are suitable for biological cells and provide the ability and flexibility to control spatiotemporal biochemical parameters. One of current efforts for biosensor technologies aims to develop sensing technology directly using small volumes of the human fluids, such as blood, urea, and saliva, for accurate, fast, and affordable detection and analysis. For example, biosensors used for earlier cancer diagnosis requires the detection of cancer biomarkers directly from small volumes of human blood $^{67}$. Figure 1.5 shows a researcher handling an integrated microfluidic device for cancer detection. The innovative microfluidic device for cancer detections can efficiently decrease the use of blood volumes in much shorter time. 


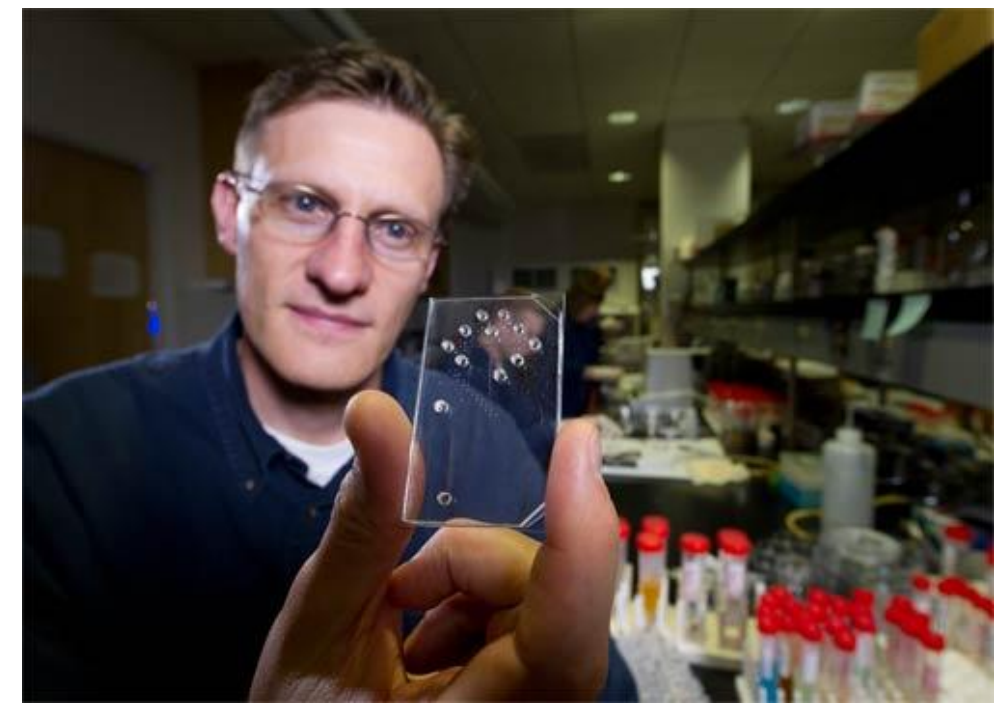

Figure1.5 A microfluidic biosensor device for cancer detection ${ }^{8}$

The advantages of microfluidic device for blood analysis are as follows: First the devices are cheap and easy to fabricate. Polydimethylsiloxane (PDMS) is the most commonly used material for microfluidic device fabrication. It is cheap and easy to fabricate compared to traditional MEMS fabrication materials. Second, microfluidic devices can handle small fluid volumes in the range of nanoliters to microliters. A few microliters of blood sample will be sufficient for detection and analysis compared with conventional approaches which require larger volumes in milliliters or liters. Third, the dimensions of microfluidic chips are generally in a few square centimeters and suitable for the development of portable sensing devices, which would be convenient for point-of-care uses. In this thesis work, we emphasis on developing microfluidic based human blood plasma separation technologies. The technologies have potential to integrate with other microfluidic functional units and sensing elements for a portal biosensor platform used for heavy metals or cancer biomarker detections. 


\subsection{Summary}

In this introduction chapter, the importance of blood analysis and blood plasma separation techniques were introduced.

In the chapter 2, detail information for conventional methods for human blood plasma separation was discussed. Compared to these methods, microfluidic methods in our research have the advantages of small blood volume handling, low cost, easy fabrication, and portable.

Chapter 3 introduced the mechanisms for microfluidic blood plasma separation. By combining three important hydrodynamic effects - bifurcation law, centrifugation force and Fåhræus-Lindqvist effect - we designed two types of microfluidic bifurcation devices.

In chapter 4, we exploited a COMSOL Multiphysics model to simulate the fluid flow in the microfluidic bifurcation devices. Detail simulation implementation was introduced.

Chapter 5 is the experimental section. The experimental setup, conditions and results for blood plasma separation were discussed in this chapter. The electrophoresis analysis of centrifuged plasma (control) and microfluidic separated plasma (test) showed that the microfluidic separated plasma has the same blood plasma 
components as compared with that in the control group.

In chapter 6, we developed a membrane based plasma separation technique. Vivid Plasma Separation Membrane from Pall life Science Corporation was directly incorporated into a microfluidic capillary device.

Chapter 7 summarized our work and provided information regarding to the future direction. The microfluidic plasma separation device developed in this thesis research can be integrated with other microfluidic units for a portal and functional lab-on-a-chip device. 


\section{CHAPTER 2: Literature Review}

\subsection{Conventional Methods}

As introduced in Chapter 1, conventional methods for cell identification and plasma separation include flow cytometer, centrifugation and filtration. The separation mechanism of flow cytometer, centrifugation, and filtration are introduced in this chapter. In addition, microfluidic techniques combined with filtration and centrifugation approaches are introduced.

\subsubsection{Flow Cytometer}

Flow cytometer for blood separation was first used in $1960^{9}$. The main principle of the flow cytometer (Figure 2.1) is first to use a laser beam of a single wavelength to suspend particles $(0.2$ to $150 \mu \mathrm{m})$ in a hydrodynamically-focused stream of liquid and a number of detectors are aimed at the point where the stream passes through the laser beam. Fluorescent chemicals in the particle or attached to the particles will be excited to emit light at a different wavelength other than that of the laser excitation. The combination of scattered and fluorescent light is detected and fluctuations in brightness are analyzed by the detectors to derive various physical and chemical information used for cell counting, sorting, biomarker detection and protein engineering. Because of its good sorting ability, flow cytometer can easily be used to separate or purify particles from solutions based on the separated particles' properties. In addition, the flow cytometer can simultaneously get multi-parametric information 
from up to thousands of particles in seconds.

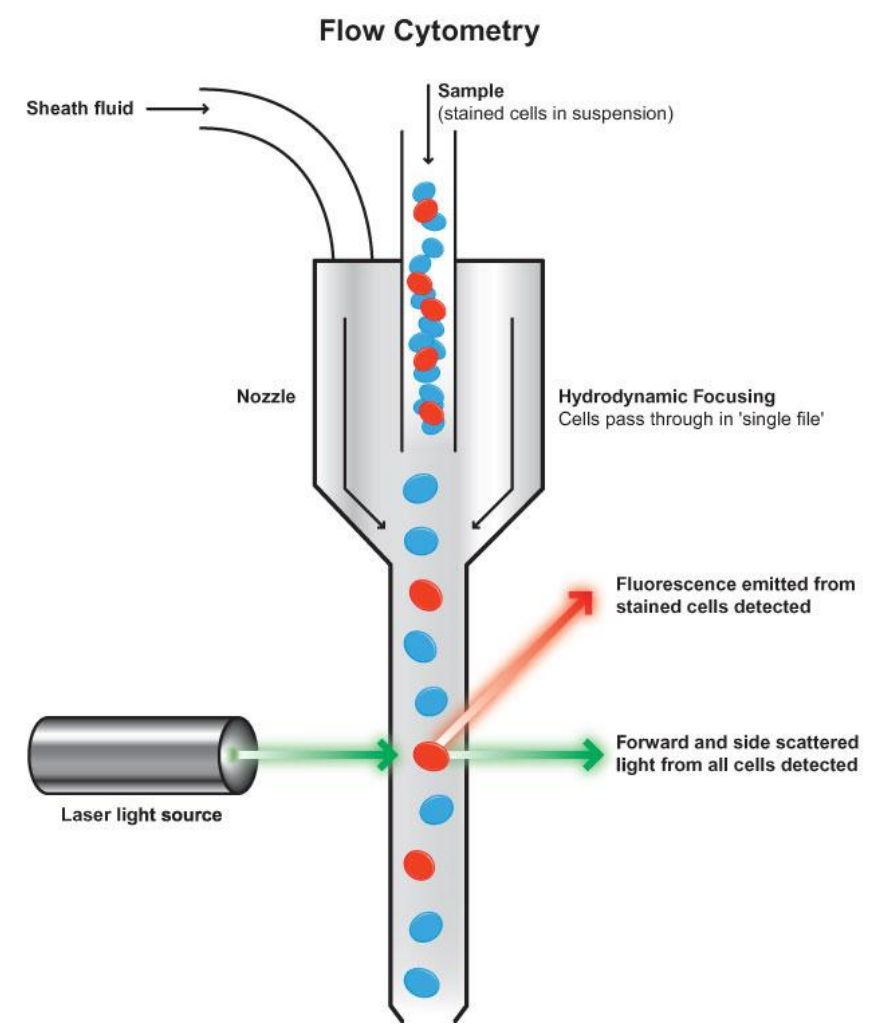

Figure 2.1 Schematic diagram of the flow cytometer ${ }^{10}$

In Terstappen's paper, they used high-resolution flow cytometer to identify and characterize the plasma cells from normal human bone marrow. The low frequency of the plasma cells and the lack of specific cell surface antigens are significant impediments for an accurate identification of plasma cells ${ }^{11}$. For this method, although it can reach fine details and high throughput, and also represent today's golden standard in cell identification and separation, the sophistication of this technique makes it challenging to be used as a convenient and portable way for blood separation. Additionally, this technique requires high levels of skill and professional handling in the lab.

\subsubsection{Filtration}


Filtration technique is another common method applied for blood separation. Moorthy et al. presented a porous filter which was embedded with a composition of a pre-polymer mixture. Their approach allowed for the filtration of cells with sizes larger than $3 \mu \mathrm{m}$ in diameters ${ }^{12}$. The porous filter was fabricated inside the channel by incorporating a photo-initiator as a part of the pre-polymer composition. Figure 2.2 (a) shows the configuration of filtration system. The microfluidic device with separation region is shown in Figure 2.2 (b). The cross section between filter channel and main channel was the separating region for blood plasma.
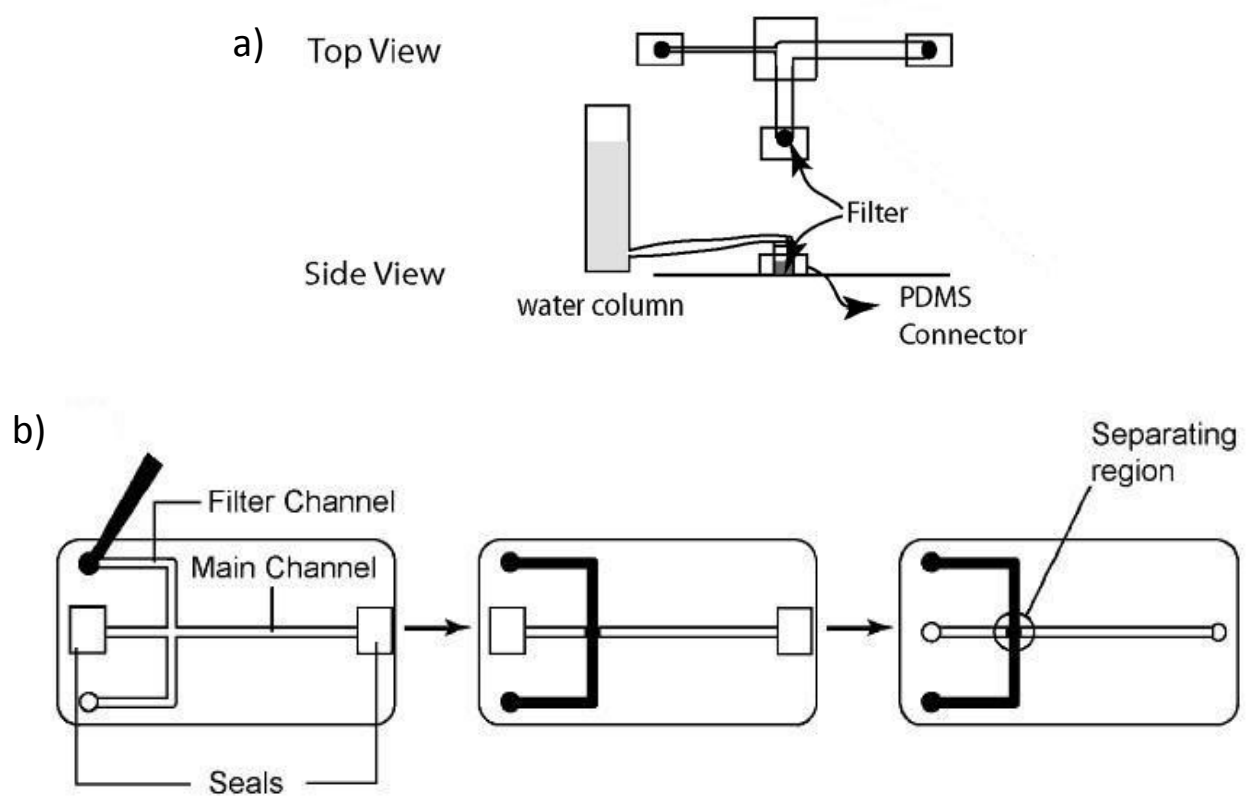

Figure 2.2 (a) The side view and the top view of a microfluidic filtration system.

(b) The microfluidic filtration device ${ }^{12}$.

In order to demonstrate the separation of porous filter for different sized particles, they used a mixture of $45 \mu \mathrm{m}$ and $3 \mu \mathrm{m}$ fluorescently-labeled beads flowing through the microfluidic device. The results showed that larger beads were held against the filter while the smaller beads can pass through the porous filter. The porosity of filter 
can be modified by varying the emulsion composition, material properties and the applied polymerization method. Hence various sized porosities of filters can be in-situ fabricated for filtration of particles with different diameters. However, this device was easily clogged because the filter's filtration capacity was limited by the size and volume holding of the filter. For example, for a cross section area of $1 \mathrm{~cm} \times 150 \mu \mathrm{m}$, about $20 \mu$ of diluted blood can be separated.

Due to its clog problem and particle trapping capacities, filtration method is not desirable for continuous separation of blood. However, for acquiring specific volumes of plasma, it can be used as a good solution. The dimensions of the device can be designed and calculated for the volume of needed plasma which was based on the Darcy's law which will be introduced in the Chapter 7 for a membrane based plasma separation.

\subsubsection{Centrifugation}

Centrifugation is a process that involves the use of the centrifugal force for the sedimentation of mixtures with a centrifuge and is commonly used in industry and in laboratory settings requiring different particle-solution separations.

Ducree and Madou presented a rotating disk to extract blood plasma by applying the centrifuge force in the device ${ }^{13,14}$. (Figure 2.3) A spinning speed with the frequency at $40 \mathrm{~Hz}$ was applied on a rotating disk for $20 \mathrm{~s}$. About $2 \mu \mathrm{l}$ plasma ( $5 \mu \mathrm{l}$ of whole blood 
was applied) can be extracted at this moderate spinning frequency. This rotating disk was also easily used as a centrifuge platform to integrate with other microfluidic components.

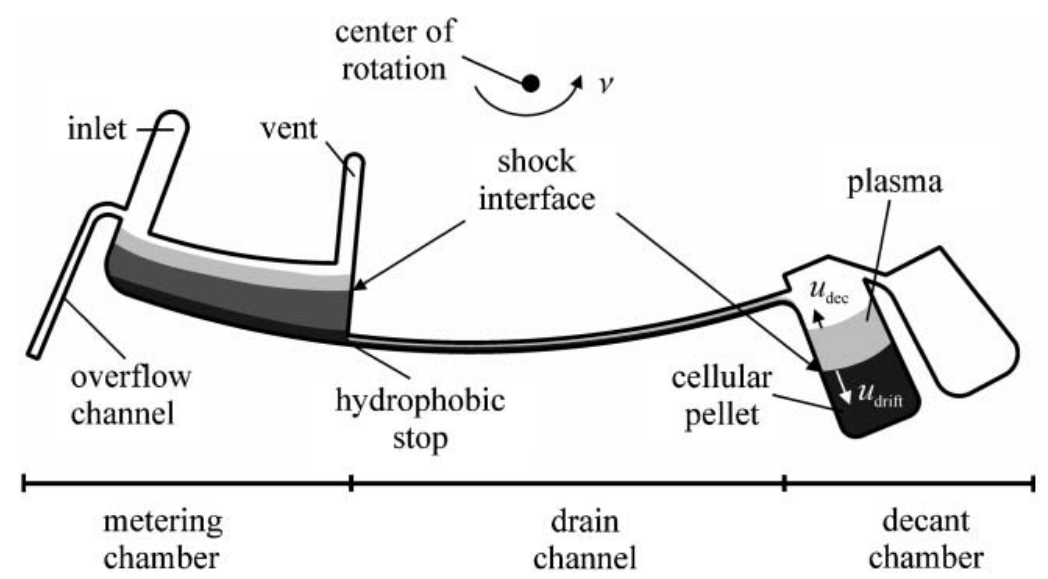

Figure 2.3 A microfluidic centrifuge device ${ }^{14}$

The experiment results showed a good residual cell concentration of only $0.1125 \%$. However, the technique was limited as a continuous blood separation method. In addition, the process was difficult for handling very small sample volumes (such as in $\mathrm{nl}$ to a few $\mu$ l ranges) because the inertial forces diminished with the reducing size of the separation channels. Moreover, centrifugation requires external power and may not be practical in a portable diagnostic device. For regular based centrifugation method, cell damage is also a concern ${ }^{15}$.

In general, conventional methods using flow cytometers, centrifugation and filtration have been widely applied from clinical lab uses for various biomedical applications, and will be continuously used as key technologies. However, they have different 
limitations which prevent them for being directly adapted as point-of-care biosensing technologies, such as requiring high level of skills to operate, external power supplies, long processing time, large volumes of solutions, and larger equipment not portable for on-site analysis. Addressing above challenges can benefit from microfluidics and lab-on-a-chip technologies, which can provide new separation and analysis methods, such as miniaturization of current bulk assays, using small volumes of samples, gentle manipulation, precise controlling microenvironment, and parallel operation and highly integration.

\subsection{Micro-scale Technologies for Blood Processing}

Microscale technologies and microfluidics have be applied in many biological and chemistry analysis, such as blood cells applications and plasma separation based on the different blood cells, their sizes and various densities, electrical permittivity, dielectric characteristics and adhesiveness. These small-scale techniques have been applied for the chip based blood separation and greatly benefit the biosensor development.

\subsubsection{Mechanical Force Based Blood Separation}

Based on the sizes of particles or cells, the micro-scaled devices can be designed to achieve the separation of blood by applying different mechanical forces. For example, it is easy to apply centrifuge forces on the cells to get the sediment. 

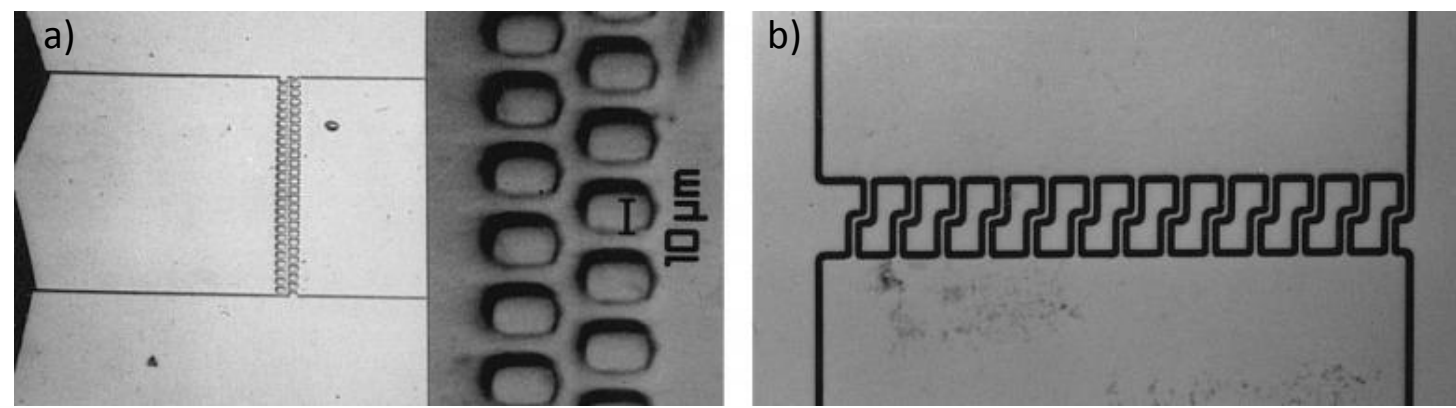

Figure 2.4 (a) Offset array of simple microposts set across a 500-mm-wide silicon channel. (b) Array of complex microposts separated by 7-mm-wide tortuous channels.

In Wilding's paper, they designed a microfluidic device embedded with micropost arrays, which were across the fluidic channel to separate white blood cells from the whole blood $^{16}$. Figure 2.4 presents two microfluidic devices with different arrays that can separate leukocytes from whole blood. The $\mathrm{T}$ shape and zigzag shape in the design can trap cells when they go through this separation region. Filtration was exploited based on the sizes of cells in these devices.
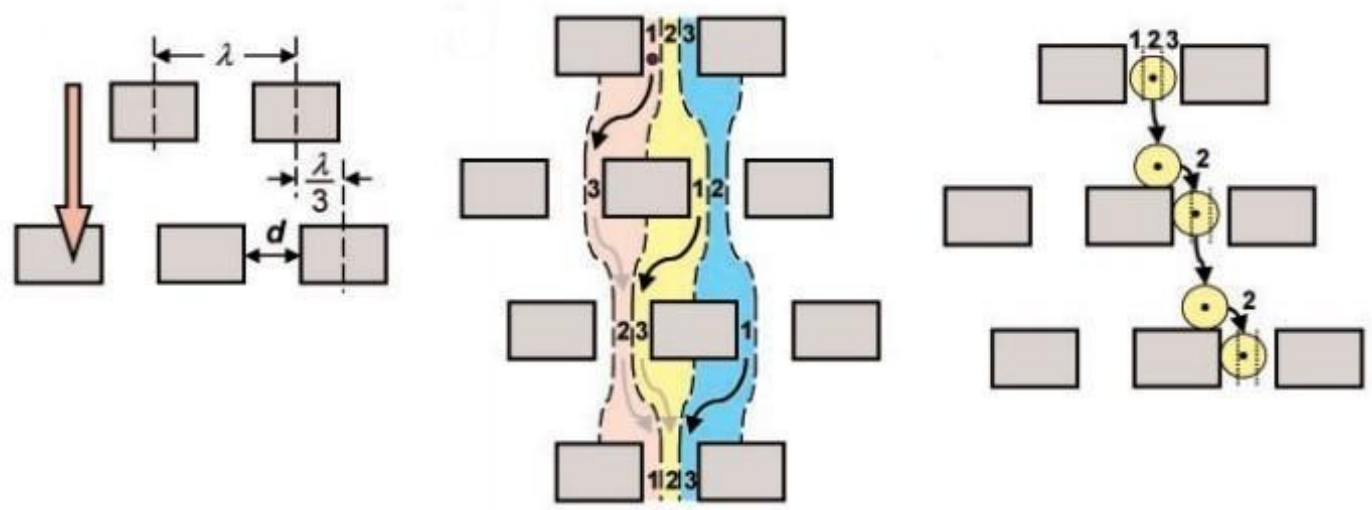

Figure 2.5 Obstacle array designs for trapping cells

Another example for microfluidic based separation was to investigate the particle movements in a laminar flow stream. In Huang's paper, they separated blood cells through differential lateral displacement of obstacles owing to asymmetric bifurcations of laminar flow around obstacles, which were designed with dimensions 
comparable to the sizes of the particles ${ }^{17}$. Figure 2.5 shows the obstacles design which can separate the particles as they passing through the structure.

\subsubsection{Dielectrophoresis Based Blood Separation}

Human cells can be polarized when they are exposed to an electric field. Dielectrophoresis, a phenomenon in which force can be exerted on a dielectric particle when it is subjected to a non-uniform electric field, can be applied to induce cell motions and separate them when they flow through the electric fields. There are several factors that influence the polarization, like bi-lipid membrane characteristics, internal structure, sizes of nucleus, and frequencies of alternative electric fields. For micro-scale technology, low voltages can be conveniently applied through the microchannels by the integration of electrodes and the microfluidic device.

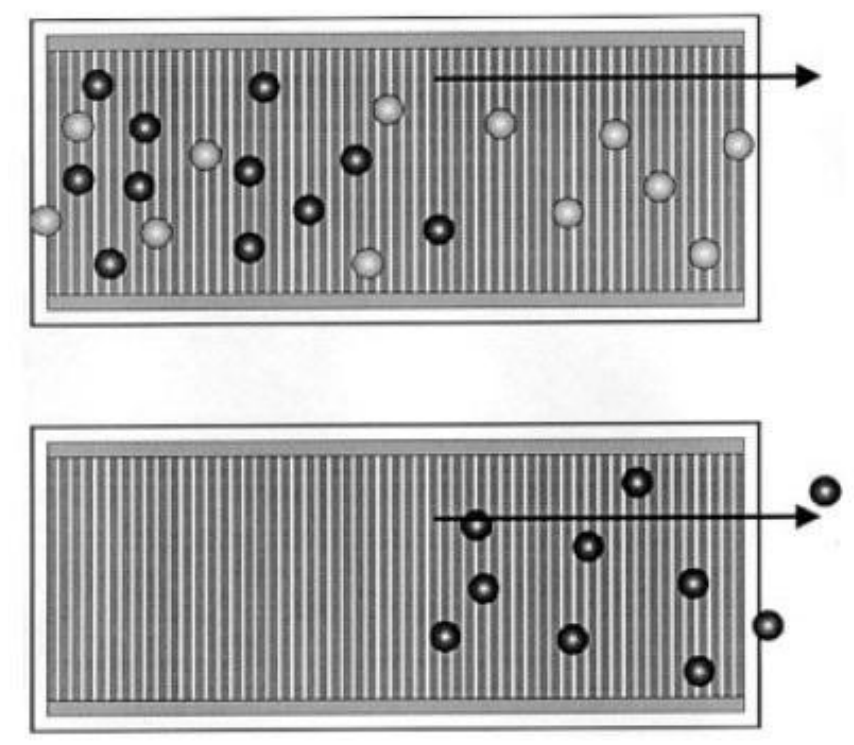

Figure 2.6 Schematic diagram of a flow separator

In Hughes's paper, the authors presented a flow separator that can separate two kinds of particles using an electrode array ${ }^{18}$. (Figure 2.6) There were two kinds of particles, 
which were light and dark particles. When the dark particles were trapped by positive dielectrophoresis, the light particles flew through the chamber and exit. Then the electric field was removed and the dark particles were release from the trap for collection. By this method, the mixture of particles can be separated through a separation chamber consisting of an electrode array at the bottom of the chamber.

\subsubsection{Optical Interaction Based Blood Separation}

The optical interaction by using a laser tweezer has been applied for blood separation devices. This technology avoids the mechanical touch between cells and surfaces. However, due to its trapping characteristics, only one cell at a time could be manipulated. In order to process a large quantity of cells in parallel, high throughput type devices should be developed.

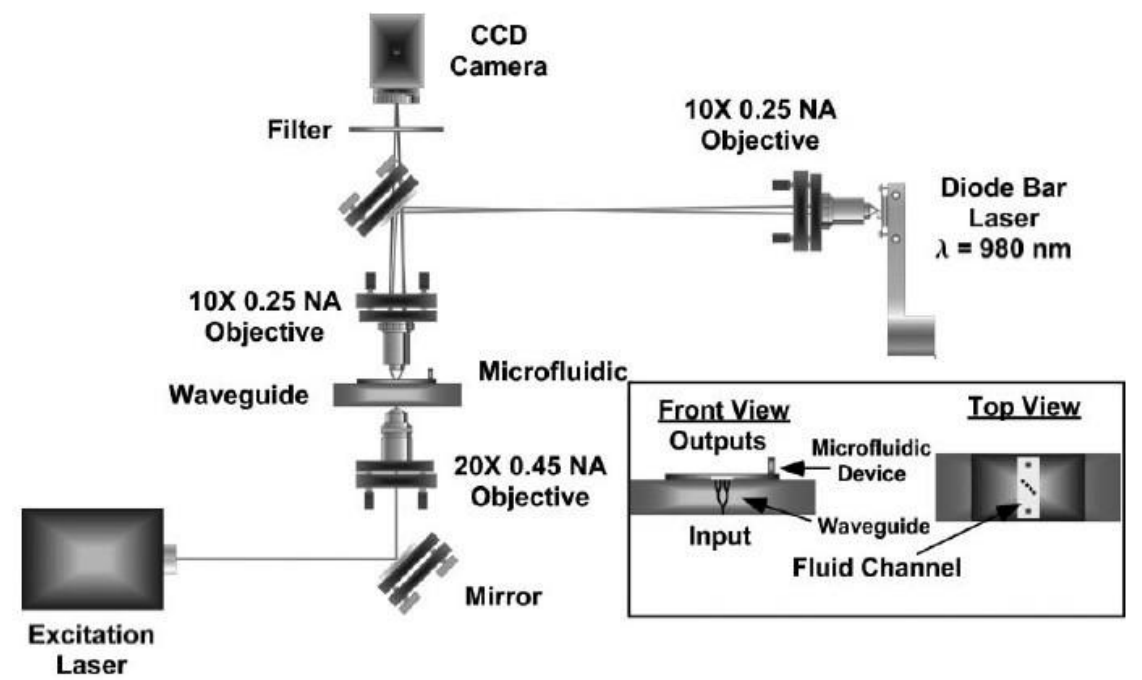

Figure 2.7 Schematic design of an integrated microfluidic sorting system ${ }^{19}$

In Applegate's paper, they presented an integrated optical platform for cells and other particles sorting in microfluidic devices ${ }^{19}$. Figure 2.7 shows the schematic design of 
the system based on optical waveguide integration with a diode laser bar trapping. The microchannel and contents were imaged using a charge-coupled device (CCD) camera connected to a monitor and a camcorder to capture the images. Two lasers exciting appropriate wavelengths were utilized for the separation of two dyed particles.

\subsubsection{Magnetic Based Blood Separation}

Red blood cells (RBCs) and white blood cells (WBCs) behavior differently under a magnetic field, in which RBCs and WBCs can be separated from each other in high magnetic gradients.

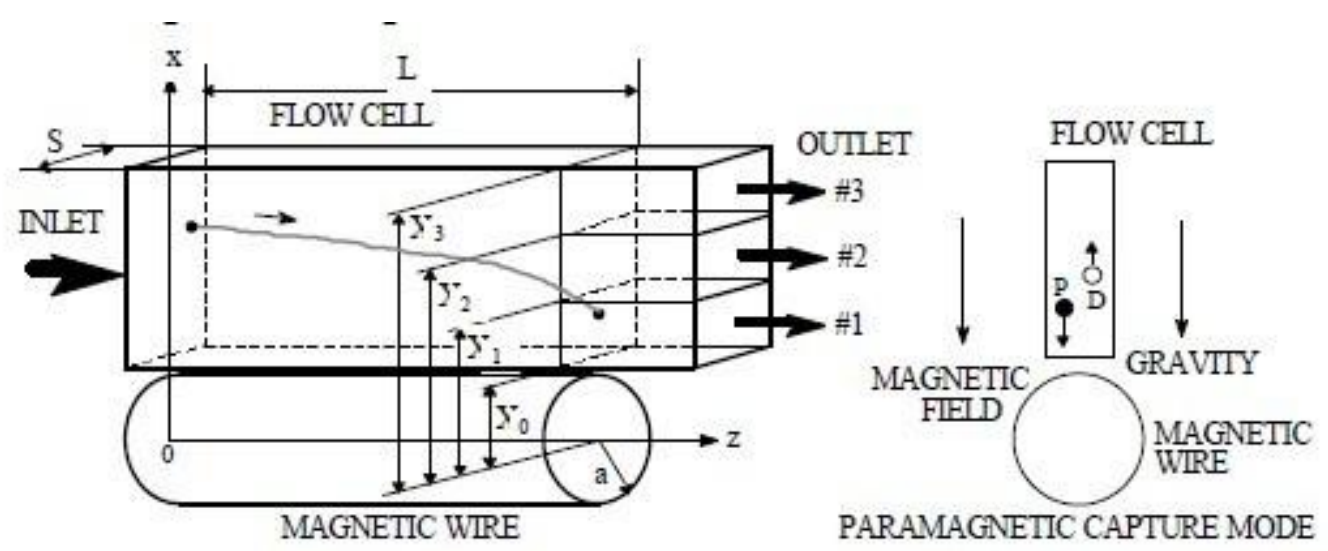

Figure 2.8 (a) Schematic diagram of a continuous magnetic separator having one inlet and three outlets. (b) Cross-section view of the separator in the paramagnetic capture $\operatorname{mode}^{20}$.

In Takayasu's paper, they used above introduced magnetic configuration to separate red blood cells from whole blood ${ }^{20}$. As show in Figure 2.8, the magnetic system had one inlet and three outlets for the cells flowing through. By controlling the flow speeds of the blood, cells and plasma can be separated into different outlets. The separation system combined magnetic force and gravity force. In the paper, they also discussed the separation of white blood cells and plasma by changing of the magnetic 
system.

\subsubsection{Biomedical Interaction Based Blood Separation}

The method of biomedical interaction is for separation of WBCs and RBCs based on their dissolve ability in a selective toxic environment. For example, WBCs are more resistant to solutions of ammonium chloride than RBCs. Figure 2.9 shows a microfluidic device for selective lysis of $\mathrm{RBCs}^{21}$.

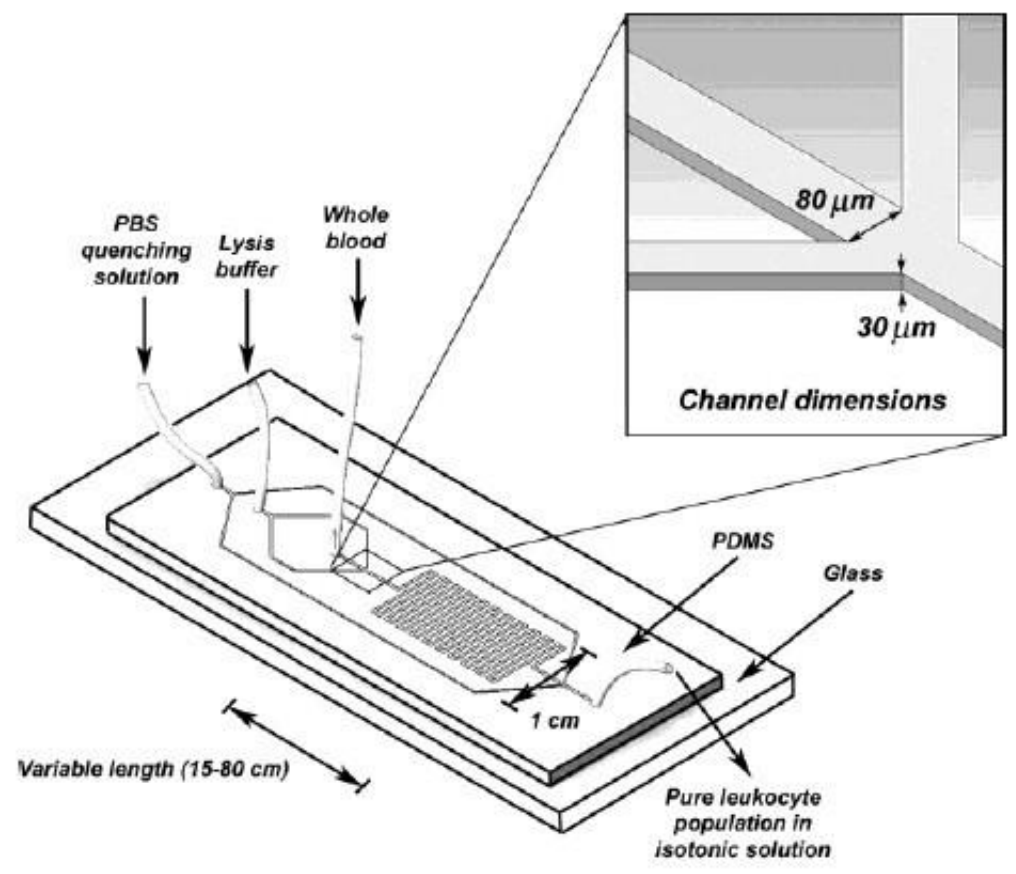

Figure 2.9 A microfluidic lysis device

Lysis buffer $\left(\mathrm{NH}_{4} \mathrm{Cl}\right.$ lysis buffer) and whole blood were mixed together in the microchannels. After the solution flowing through the microchannels with a very long length $(20 \mathrm{~cm})$, complete erythrocyte lysis was achieved. The whole process takes about 10 minutes. Both rat blood and human blood have been used in the experiment and $100 \%$ lysis was achieved.

\subsection{Summary of the Microfluidic Methods}


In above sections, we introduced microscale separation technologies, and the examples showed that microfluidics have been applied with many other different separation mechanisms involving mechanical forces, optical interactions, fluid dynamics, and chemicals.

The history of microfluidics can be traced back to earlier of 1950s. The efforts to dispense small volumes of liquids in the nanoliter and sub nanoliter ranges were developed and as the foundation for today's ink-jet technology. Today, the impact of microfluidic technologies has dramatically increased during the past two decades. Microfluidics has been applied to many different areas, such as biotechnology and biosensor, diagnostics, medical applications, and pharmaceutical industries. More and more microfluidic components have been developed for fluid transport, metering, mixing $^{22}$, valves ${ }^{23}$, pumping $^{24}$, concentration or separation of molecules within very small volumes of fluids in the last two decades.

Blood cell analysis for infectious diseases, cancers, and inflammatory responses can be conveniently done at homes or in doctors' offices for rapid and accurate diagnosis and prognosis. Microfluidics and miniaturized lab-on-a-chip (LOC) devices would be attractive approaches in these medical needs for blood analysis. In drug delivery, microfluidic devices may redefine the entrance criteria for clinical trials and test of these criteria in a time- and cost-effective way ${ }^{2}$. 
The microfluidic based methods have many advantages. 1) Miniaturization of current bulk assays. Unlike traditional methods, several microliters of samples would be enough for the detection. 2) It can prevent or reduce the cell damage. Traditional methods, like centrifugation or filtration, can introduce blood hemolysis during operation because of intense external forces or resistances, which can cause the rupturing of erythrocytes and releasing of some components into the surrounding fluids. 3) Microfluidics is highly integrated. As we mentioned above, many different micro components are available for integrate functional units together in a very small chip (such as several square centimeters) to achieve complex tasks. 4) It is low cost and easy to fabricate. PDMS is primary material and commonly used for microfluidic device fabrication. PDMS has many advantages, such as inert, non-toxic, non-flammable and biocompatible. 5) Microfluidics can provide a faster and automatic processing approach compared with other methods. Traditional methods require high levels of skills to handle the machines and long processing time. Microfluidic method is faster and easily used as portable analysis technique.

The microfluidic method we used for blood separation applied with several hydrodynamic effects, such as the bifurcation law, centrifugation force and Fåhræus-Lindqvist effect. The main principle for the blood separation is the bifurcation law. It reveals that the cell behavior when they flow through a bifurcation region in a microchannel. Centrifugation force is also applied in our design to form cell-free layer in the bifurcation region and increase the separation rate. The 
Fåhræus-Lindqvist effect deals with the viscosity change due to the change of channel diameters. We combined these three hydrodynamic effects to maximize the separation efficiency and plasma yield.

In the Chapter 7, we investigated membrane based microfluidic plasma separation. The advantages of this method are 1) passive plasma separation, 2) no extra power required, 3) avoid complex microchannel designs, and 4) achieve high plasma separation efficiency and yield. It is similar to filtration method as we discussed above. In general, we fabricate two layers of PDMS channels and bonded them together embedded with the membrane in between. Because of the resistance property, the varied porosity of the membrane, and capillary driven force, the membrane can trap the blood cells and the plasma can be extracted. 


\section{CHAPTER 3: Principles for Microfluidic Based Plasma Separation}

\subsection{Hydrodynamics of Blood Cells}

RBCs can behave differently and change their morphologies within the fluids with varying Reynolds numbers $(R e)$ and different degrees of shear forces. There are generally three types of movements for the cells in microchannel, including tank-treading, swinging and tumbling as listed in the following Table $3.1^{25}$.

Table 3.1 Illustration of the different regimes of cell behavior in a microchannel with dimensions below $300 \mu \mathrm{m}$

\begin{tabular}{|c|c|c|c|}
\hline Movement of cells & Reynolds numbers & Viscosity ratio & Predominant force \\
\hline Tank-treading & Very low $(<1)$ & Low & Viscous lift force \\
\hline Swinging & Low & Medium & Transition regime \\
\hline Tumbling & Very low to low $(\sim 1)$ & High & Inertial lift force \\
\hline
\end{tabular}

The above table shows different movements of the cells and their corresponding responses to the different ranges of $\mathrm{Re}$, viscosity ratio and predominant forces. The viscosity ratio is the ratio between the inner fluid of RBCs and the surrounding media solution. For tank-treading movement, the cell membrane can rotate around its center of the mass, and the cell maintains a stationary orientation with the flow ${ }^{26}$. At a relatively high Re and viscosity ratio within the laminar regime, $\mathrm{RBCs}$ can flip around 
themselves, and this movement is often referred as tumbling. The third movement as mention above is swinging, which is a transitional regime. In this situation, the cell tank-treads but undergoes oscillations around its stationary orientation ${ }^{27}$. The cell keeps a fixed orientation with the tank-treading regime. Research of these cell movements is of great helpful to design the patterns of microfluidic devices used for the blood cell separation.

\subsection{Bifurcation Law (Zweifach-Fung Effect)}

An important regulation principle related to blood cells' behavior inside a microfluidic channel is the bifurcation law, which was derived from in vivo microcirculation. When the blood cells flow through a bifurcating region of the blood vessel, they have a tendency to travel into the daughter vessel which has a higher flow rate and leave very few cells flowing into a lower flow rate vessel ${ }^{28}$.

Figure 3.1 (a) shows a schematic diagram for interpreting the bifurcation law. A blood cell enters the microchannel from the left side and reaches to a bifurcation region where two downstream branch channels meet. Due to their geometric difference, these two daughter channels have different flow rates. We assume the upper branching channel has higher flow rate than the lower branching channel. According to the bifurcation law, this cell will flow into the upper channel. The pressure gradient in the upper channel is usually higher than that in the lower channel, which results in an asymmetric distribution of the shear rate along the microchannel at the bifurcation 
region. The different distribution of shear rates along the channel can exert different shear forces on a cell. Larger shear forces are generated along the upper channel (high flow rate) compared with the lower channel (low flow rate), and the cell is pulled towards the upper channel. The illustration of shear forces on the cell body is shown in the Figure 3.1 (a).
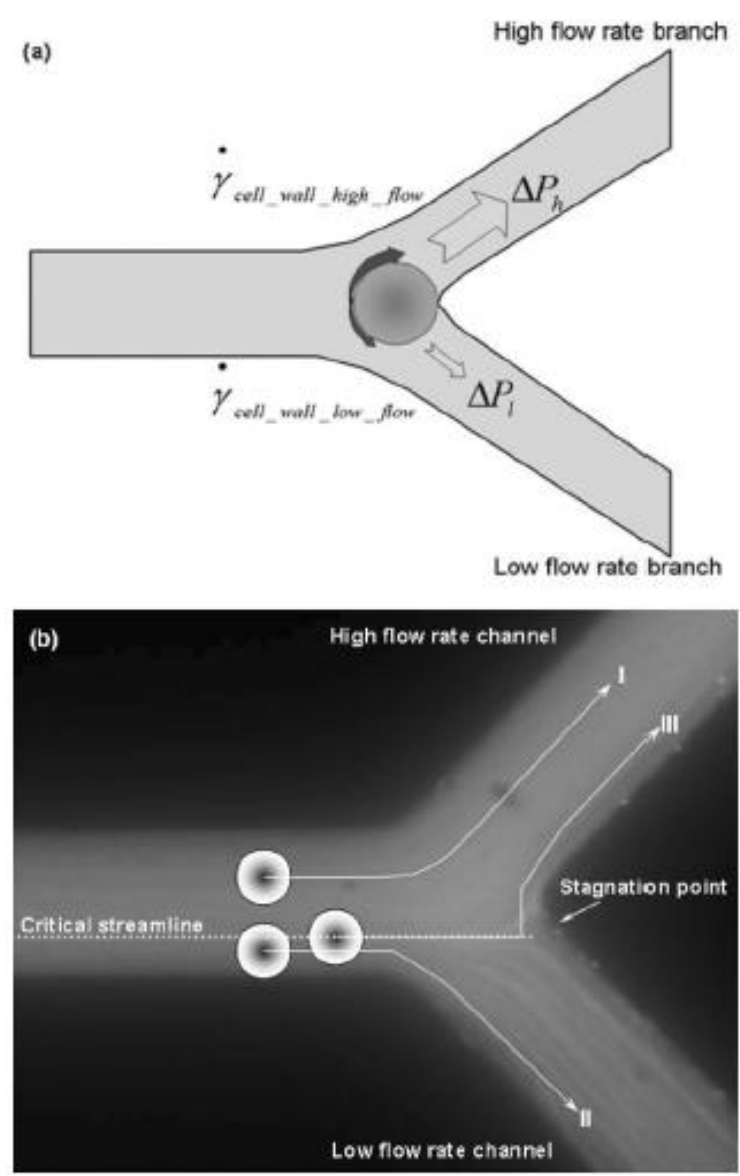

Figure 3.1 (a) Schematic diagram for interpreting the bifurcation law. (b) An illustration of critical streamline ${ }^{28}$.

In an ideal case, the critical flow rate ratio for the blood separation is 2.5:1 when the cell-to-vessel diameter ratio is of the order of one. A critical streamline can be determined by the flow rate ratio of two daughter channels. Figure 3.1 (b) illustrates the critical streamline of blood separation. In this case, when the centroid of cell is 
above the critical streamline, such as the cell I in Figure 3.1 (b), the cell will flow into the high flow rate channel. If the cell's centroid is underneath the critical streamline, such as the cell II in Figure 3.1 (b), the cell will flow into the low flow rate channel. If the cell's centroid is on the critical streamline, such as the cell III in Figure 3.1 (b), the cell will still travel into the high flow rate channel. If the cells distribution is uniform through the whole channel, the critical streamline should be kept close to the sidewall of the low flow rate channel to achieve high separation efficiency.

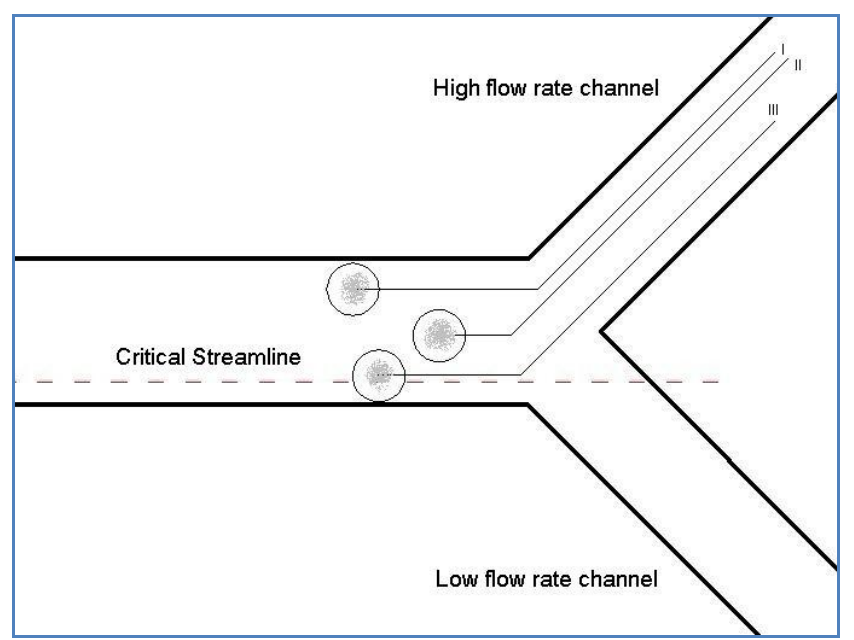

Figure 3.2 Schematic diagram for an example with a 100\% separation

If the flow rate ratio is large enough, the distance between the critical streamline and the sidewall will be smaller than the radius of the cell as shown in Figure 3.2. In this case, because all cells' centroids are above the critical streamline, all of the cells will flow into the upper high flow rate channel and the separation efficiency can reach to $100 \%$.

\subsection{Centrifuge Force}

Although the bifurcation law is an important mechanism for blood separation, other hydrodynamic effects can also influence the separation and vary the efficiency. In 
Menz's paper, a bend channel structure in a microchannel can assist the plasma separation as shown in the Figure 3.3 $3^{29,30}$.

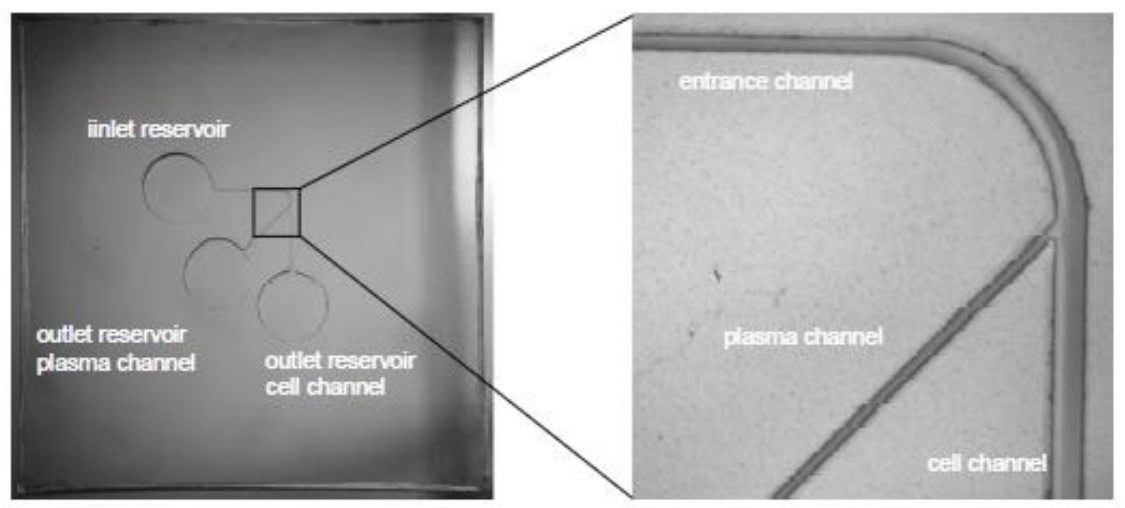

Figure 3.3 Microfluidic bend channel structure for assisting blood separation

This separation unit consists of a simple microchannel design with a branching channel followed after a bending channel structure. The device has one inlet reservoir for the blood input, one outlet reservoir for the separated plasma and another outlet reservoir for the blood cells collection. The bend channel was designed to close to the bifurcation region and apply centrifugation forces on blood cells. In this case, before blood cells flew through the bifurcation region, the bend structure exerted centrifugation forces on the blood cells and forced the cells to travel along the outer side of the main channel. The fluid with a cell-free layer was generated along the inner side of the channel and thus improved the separation efficiency.

They also examined the effects of the bend channel positions relative to the bifurcation on the separation efficiency. The results showed that the separation efficiency slightly increased when the angles of the bending structure was increased because larger centrifuge forces were exerted on cells. In our microfluidic device 
design, we also considered to use the bend channel structure to improve the separation efficiency.

\subsection{Fåhræus-Lindqvist Effect}

Fåhræus-Lindqvist effect is also an important factor that needs to be considered for designing the microfluidic device for blood cell separation. The Fåhræus-Lindqvist effect is an effect that the viscosity of the blood can change with the vessels' diameters as the blood flows through, such as the blood viscosity is decreased as the vessel's diameter decreases. This is because erythrocytes move over the center of the vessel, leaving plasma at the wall of the vessel ${ }^{31}$. In other means, the Fåhræus-Lindqvist effect states that the viscosity of the blood is not a constant quantity, but depends on the diameters of the vessels ${ }^{32}$, especially when the vessels' diameters are ranging from $10 \mu \mathrm{m}$ to $300 \mu \mathrm{m}$.

For our microfluidic device, the channel dimensions are varied from $20 \mu \mathrm{m}$ to $200 \mu \mathrm{m}$, Fåhræus-Lindqvist effect should be considered regarding to the separation efficiency. In addition, in numerical simulation, the fluid property, such as the viscosity, should be considered for approximate to the real blood flow situations. 


\section{CHAPTER 4: Microfluidic Device Design and Fabrication}

\subsection{Single Bifurcation Microfluidic Device}

A good understanding of the hydrodynamic laws and effects (Chapter 3) for separation of blood cells is very important for designing the microfluidic device. In order to maximize the efficiency of the blood plasma separation, we considered the bifurcation law and Fåhræus-Lindqvist effect and applied a bending structure for the generation of centrifugation force.

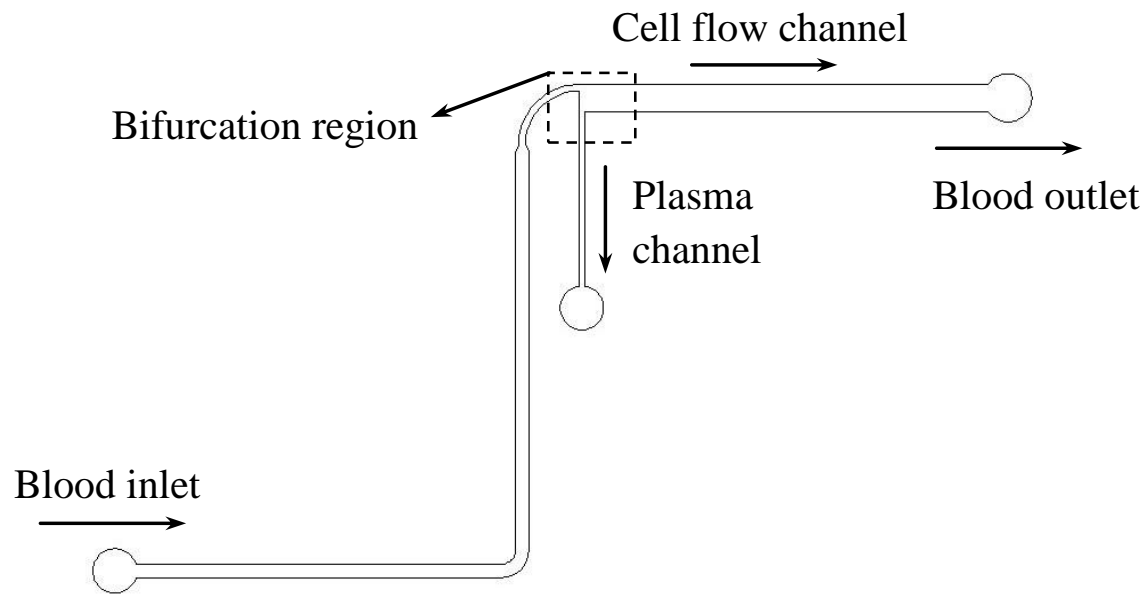

Figure 4.1 The schematic design for microfluidic single bifurcation device

As shown in Figure 4.1, a single bifurcation microfluidic device was designed for the blood separation. This design was inspired from the design in Huang's paper especially for the bifurcation region ${ }^{33}$. The curvature structure, also working as a constriction channel, and expansion channels were exploited in the device design as shown in the bifurcation region (dotted frame in Figure 4.1). The device had one 
blood inlet and two outlets, in which one was for the plasma output and the other was for the blood cells' collection. The whole size of the pattern was about $5 \mathrm{~mm} \times 10$ mm. The width of main channel (Cell flow channel) was $200 \mu \mathrm{m}$ and the daughter channel (Plasma channel) was $40 \mu \mathrm{m}$. For the constriction region, the width of constriction channel was $50 \mu \mathrm{m}$. The radius of the curvature channel was $400 \mu \mathrm{m}$.

\subsection{Fabrication}

The basic idea of microfluidic device fabrication is to transfer the designed patterns which were drawn in the AutoCAD to actual structures. First, specific microfluidic channels were drawn in an AutoCAD file, and the designed patterns were printed onto a mask, which had clear and dark regions according to the design. Second, the mask patterns were transferred into a solid master mold by photolithography process. The detailed fabrication steps were discussed in the following sections. Finally, this master mold fabricated by photolithography was used to replicate the device into a polymer material by soft lithography. PDMS elastomer is one of the most commonly used materials for this soft lithography process. (Figure 4.2)

PDMS has many advantages. PDMS is much cheaper than traditional microfabrication materials, such as glass and silicon. The soft lithography micro-molding processes are simple and rapid compared with conventional microfabrication procedures, such as etching, deposition and bonding approaches ${ }^{34}$. PDMS is transparent and has very good optical properties for the observation of 
biological experimental results under optic microscopes. In addition, PDMS is gas permeable and biocompatible. For biological experiments, especially for cell applications, the gas (oxygen) permeability is very important for keeping cells healthy in the devices during long-term experiments. In another case, sometimes air bubbles in the microchannel can increase the flow resistance and cause the flow stopped. Due to the gas permeable, the air bubbles can escape through the PDMS layer and be eliminated from the channels by applying pressures on the fluid and get rid of air bubbles.

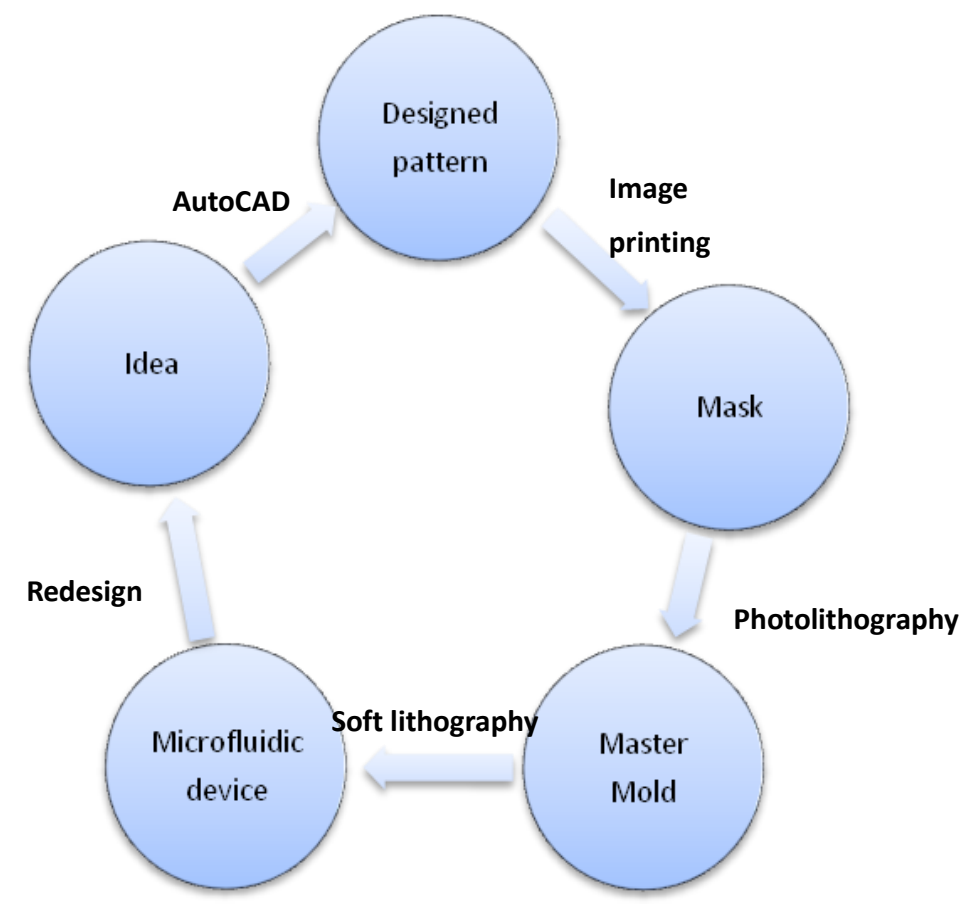

Figure 4.2 Schematic process for microfluidic device fabrication

For the master mold fabrication, initially a single-side polished 3 inch silicon wafer was cleaned with acetone and methanol, respectively. The substrate wafer was baked and dehydrated on a hot plate at $120{ }^{\circ} \mathrm{C}$ for 30 mins. In order to obtain a $20 \mu \mathrm{m}$ thickness of the photoresist layer, SU-8 2025, a negative tone photoresist, was spun 
onto the wafer at $4000 \mathrm{rpm}$ for 30 secs. After the spin coating, the wafer was soft baked on the hot plate at $65{ }^{\circ} \mathrm{C}$ for 3 mins and $95{ }^{\circ} \mathrm{C}$ for 7 mins, respectively. Then, the resist layer was exposed by a UV flood exposure system (OAI model 150) at an energy density of $15 \mathrm{mw} / \mathrm{cm}^{2}$ for 15 secs. The exposed resist was post-baked on the hot plate at $65{ }^{\circ} \mathrm{C}$ for 2 mins and $95{ }^{\circ} \mathrm{C}$ for 5 mins, respectively. After the post exposure baking, the resist was developed by SU-8 developer for 5min. The final step was the hard baking of the developed structures at $160{ }^{\circ} \mathrm{C}$ for 30 mins to assist the resist adhesion to the substrate. The pattern on the substrate was used as the master mold for soft lithographic replication of the PDMS devices.

For the microfluidic device fabrication and soft lithography process, PDMS solutions were prepared at the ratio of 10:1 (base:curing agent) and cast onto the master mold. The PDMS layer together with the master mold were baked at $60{ }^{\circ} \mathrm{C}$ for $3 \mathrm{hrs}$ to cure the PDMS, and then, the PDMS layer was peeled from the mold and holes were punched through the inlets and outlets. The punched PDMS layer was treated with 30 secs oxygen plasma and bonded with a pre-cleaned glass slide to completely seal the channel. The fabricated device was ready for the experiment.

\subsection{Blood and Device Preparation}

Whole blood was purchased from Innovative research, Inc., MI, USA and Ethylenediaminetetraacetic acid (EDTA) was used as an anticoagulant to keep the blood from clogging. First, the blood was diluted with $1 \mathrm{X}$ phosphate buffered saline (PBS) to various hematocrit levels from $15 \%$ to $45 \%$. For the hematocrit level of $45 \%$, 
the blood was still kept as a whole blood condition without any dilution. Before the experiment, the tubing and microfluidic device were pre-wetted using $1 \%$ bovine serum albumin (BSA) solution for 1 hour at room temperature. The BSA solution can keep the blood from clogging inside of the microchannels.

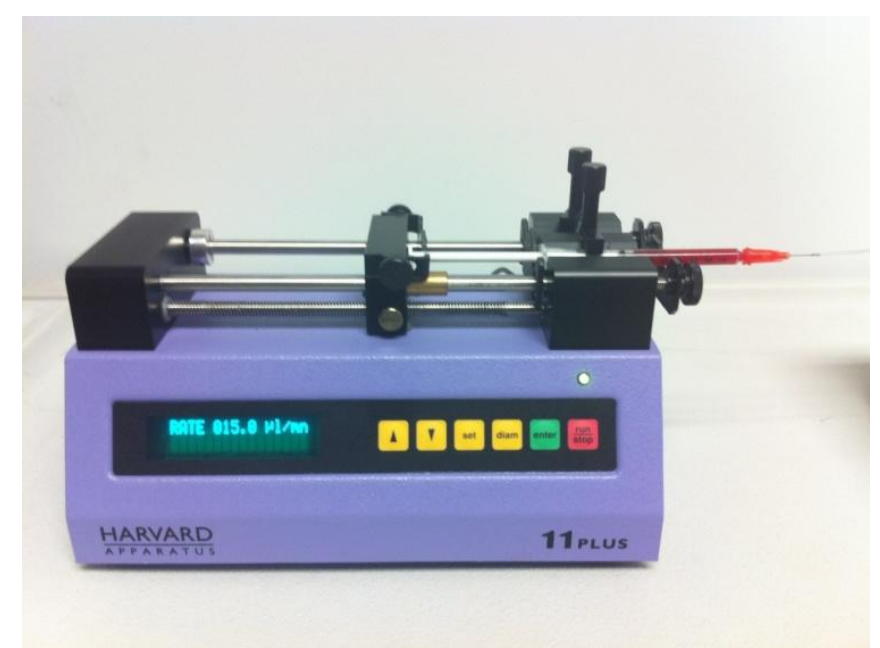

Figure 4.3 The picture shows a syringe loaded with blood hooked on a syringe pump

During the experiment, the syringe pump was set at the different running flow rates varied from $5 \mu \mathrm{l} / \mathrm{min}$ to $15 \mu \mathrm{l} / \mathrm{min}$. The plasma separation efficiency and yield were investigated under different hematocrit levels and running speeds. The images were taken by an inverted microscope (Nikon Eclipse Ti, Japan).

\subsection{Results and Limitations}

During the experiment, blood first flew through the constriction/curvature channel, the viscosities of the blood decreased as the blood flew from a wider channel in a narrow channel, simultaneously, the curvature channel exerted a centrifugation force on the blood cells. When the blood reached to the bifurcation region, a layer of blood 
cells flew along the outer edge of the channel and a layer of plasma was along the inner edge of the channel. In addition, the decreased blood viscosity in the curvature channel would assist the cells moving towards the outer edge of the channel because it was relative easier for the cells to overcome the viscosity force. Because of this phenomenon, most cells continuously flew through the main channel and plasma was separated and collected through the output of the narrow daughter channel.
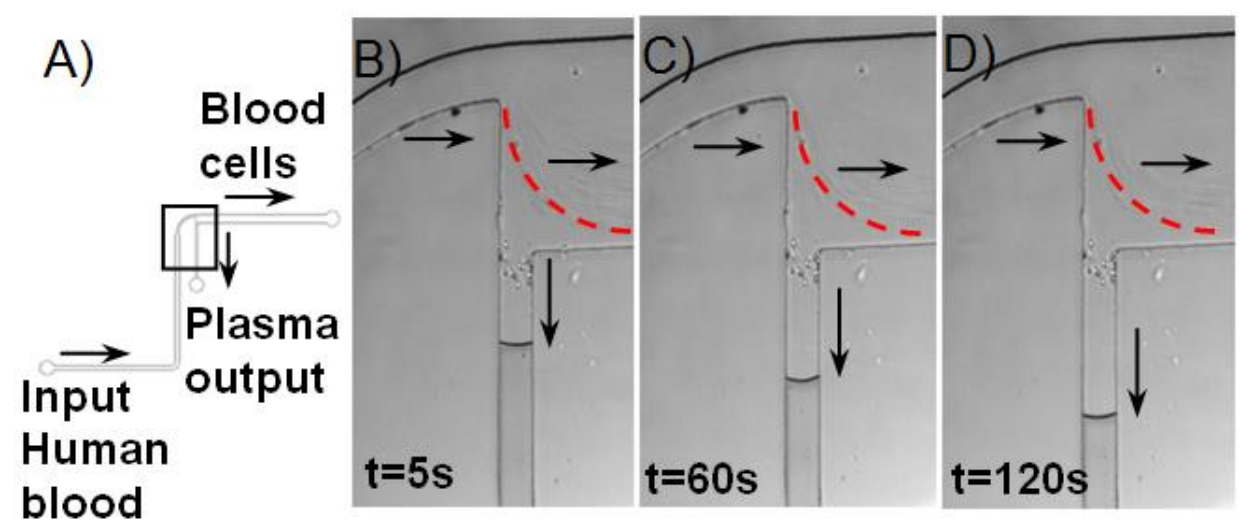

Figure 4.4 Results of single bifurcation device A) Design 1 for the microfluidic device. The frame represents the blood plasma separation region in B, C, and D. B)-D) show the blood separation at different time as indicated in the figures. The blood flow rate was kept at $3 \mu 1 / \mathrm{min}$ and the red dashed curve indicated the path of blood cells in the wider channel.

The above figures show the experimental results for the microfluidic single bifurcation based human blood separation. As shown in the Figure 4.4, the blood was separated at the bifurcation after flowing out of the curvature channel. The trajectory of the blood cells was marked with the dashed red line in each figure and indicated that the blood cells flow through the main channel and blood plasma was down into the narrow channel. 
However, we found several limitations for the single bifurcation device for the blood separation. The first problem was that the blood was hard to flow through the outlet when the device was not pre-wetted. We can solve the problem by pre-wetting the device. Another limitation was that the plasma yield was relative low. After calculation, the yield of this single bifurcation was about $5 \%-8 \%$. In order to increase the plasma yield and the separation efficiency, we designed the multiple bifurcations device pattern assisted by a COMSOL simulation model, which was discussed in the following chapters. 


\section{Chapter 5: Microfluidic Multiple Bifurcation Device and COMSOL Simulation}

\subsection{Multiple Bifurcation Device Design}

To increase the plasma separation efficiency and yield, which are the limitations of the single bifurcation microfluidic device, we designed a six-bifurcation device. Large reservoirs for the plasma and blood cells collection were design for assisting the outflow. To prevent the collapsing of these large reservoirs, small pillars were designed to support the top PDMS layer as shown in the Figure 5.1. The fabrication of this device followed the same procedures as discussed in Chapter 4.

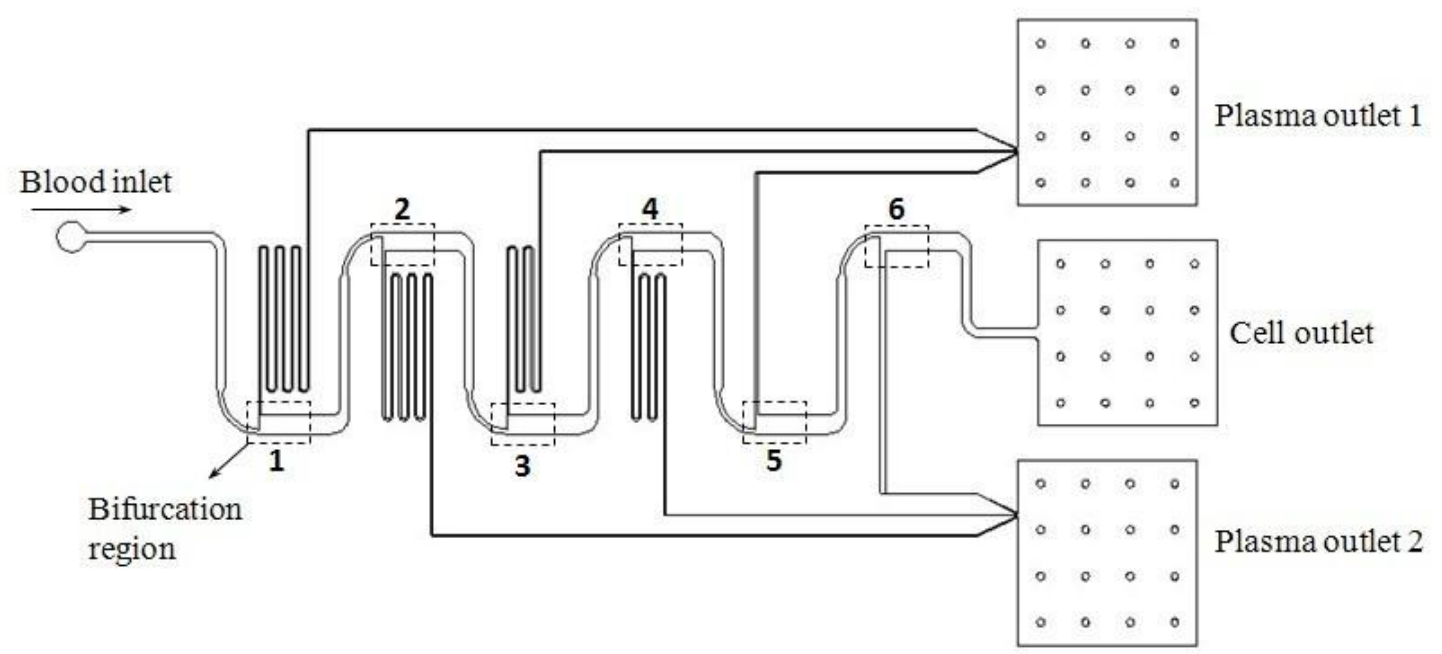

Figure 5.1 The schematic design for microfluidic multiple bifurcation device

As we discussed in Chapter 3, bifurcation law effect governs the plasma separation because of the combination of different flow rates and shear forces at the bifurcation regions. According to the law, the flow rate is related to the channel dimensions and 
the pressure differences along the channel. As indicated in Equation 5.1, $Q$ is the flow rate of the fluid. The relation between the pressure difference and the flow rate is

$$
\triangle P=Q R
$$

Where $\Delta P$ is the pressure difference and $R$ is the resistance. For rectangular channel, the resistance is a function of channel parameters as indicated in Equation 5.2:

$$
R=\frac{12 \mu L}{w h^{3}}
$$

Where $L, w, h$ is the length, width and height of the channel, respectively, and $\mu$ is the fluid viscosity. For a multiple bifurcation device, the flow rate ratio of each bifurcation can be modified by changing the dimensions of main channel and each daughter channels, such as the channel widths and lengths, which result the changes of channel resistances. If the flow rate ratio at each bifurcation was kept as the similar value, the separation efficiency would be expected to be similar.

In addition, the bending structure introduces centrifuge forces which can exert on the cells in assisting to create a cell-free plasma layer. The Fåhræus-Lindqvist effect results in viscosity changes in the microchannels with different widths. For the multiple bifurcation channel design, we considered those effects and addressed the problems found during the experiments with the single bifurcation device. We design several different multiple bifurcation devices with different channel dimensions at the bifurcations in considering of the different flow rate ratios. Although the photolithography and soft lithography techniques provide flexible and fast fabrication 
approaches, it is still challenging to test each device by experiment, which is time consuming and costly.

A simplified COMSOL Multiphysics simulation model was developed to examine the flow velocities and flow rate ratios. Different bifurcation pattern designs with varied dimensions were simulated before we went further for microfabrication and blood separation experiments. By using the COMSOL simulation, it is convenient for us to optimize the design instead of performing too many experimental tests. The detail of the COMSOL simulation was discussed in the following sections.

\subsection{COMSOL Multiphysics Simulation}

COMSOL Multiphysics Software (Version 4.0.0.982, COMSOL Inc. USA) is a finite element analysis, solver and simulation software / FEA Software package for various physics and engineering applications, especially for coupled phenomena and multiphysics ${ }^{35}$.

COMSOL has an interface to AutoCAD and it is convenient to import the designed pattern drawn in AutoCAD into the COMSOL platform. Users can directly modify the pattern in AutoCAD without rebuilding the model in the COMSOL. The COMSOL has many toolboxes, such as structure mechanics module, acoustic module and other modules for multiphysical problems. We used the computational fluid dynamics (CFD) module to build the model. This module contains different fluid flow definitions, such 
as laminar flow, turbulent flow, swirl flow and other hydrodynamic flows. The Reynolds numbers for microfluidics are always falling into a small range, which is typically small than 1 based on the channel dimensions. The fluid flow in our device was assumed as laminar flows.

\subsubsection{Implementation of the Simulation}

Blood is normally considered as a Non-Newtonian fluid, because of a nonlinear relationship between stress and rate of strain. In Xue's paper, they developed both models for Newtonian and Non-Newtonian fluids by simulating horse blood (Newtonian fluid) and human blood (Non-Newtonian fluid). From their results, the flow rate ratios of newtonian fluid was twice of that for the nonnewtionian fluid as shown in Table $5.1^{36}$. Their model includes 15 bifurcations.

Table 5.1 Flow rate ratios at bifurcations for Newtonian and Non-Newtonian fluid

\begin{tabular}{cccc}
\hline \multirow{2}{*}{$\begin{array}{c}\text { Bifurcation } \\
\text { number }\end{array}$} & $\begin{array}{c}\text { Newtonian } \\
\text { flow }\end{array}$ & & $\begin{array}{c}\text { Non-Newtonian } \\
\text { flow }\end{array}$ \\
\cline { 2 - 2 } & Human/horse & Human blood \\
\hline 1 & 25.89 & & 14.02 \\
3 & 27.06 & & 14.44 \\
4 & 28.95 & & 15.19 \\
5 & 30.02 & & 15.44 \\
6 & 33.12 & & 16.75 \\
7 & 35.12 & & 17.55 \\
8 & 37.65 & & 18.49 \\
9 & 41.62 & & 20.16 \\
10 & 45.73 & & 21.81 \\
11 & 52.63 & & 24.84 \\
12 & 62.51 & & 29.05 \\
13 & 74.94 & 34.63 \\
14 & 94.47 & \\
15 & 128.7 & \\
\hline
\end{tabular}

Some other groups have been considering blood as a Newtonian fluid to simplify the 
simulation. In this manner, the viscosities and velocities of the blood as well as the shear stresses in the main channel and daughter channels would be assumed the same. In Yang's paper, the blood is assumed to be a homogenous Newtonian fluid because blood behaves as a Newtonian fluid in high shear rate conditions ${ }^{28}$. We admitted that a Non-Newtonian fluid model can simulate the results more accurately. However, to simply the simulation, we considered the blood as a Newtonian fluid as reasonable according to other's previous work. A comprehensive simulation model is beyond the scope of this thesis work. In this case, we set the fluid viscosity to be 0.0035 pa $\bullet$ both in the main channel and daughter channels. The simulation procedures are listed in the following:

1. The fluid dynamics analysis considers the solution of the 2D Navier-Stokes equations in a stationary case and the fluid flow is a laminar flow.

2. Import the designed paattern in AutoCAD into the COMSOL.

3. Set the initial conditions and boundary conditions of the model. For initial conditions, the input fluid velocity was set as $0.05 \mathrm{~m} / \mathrm{s}$, which equals to $360 \mu \mathrm{l} / \mathrm{min}$ for the flow rate. The flow velocity can be changed to examine the influnce of velocity on the flow rate ratios. The output pressure was set as the atmopheric pressure. The flow was considered as an incompressible flow. The viscosity and the density of blood was set as $0.0035 \mathrm{pa} \bullet \mathrm{s}$ and $1060 \mathrm{~kg} / \mathrm{m}^{3}$, respectively.

4. The meshing was realized using the free triangular function that automatically created an unstructured triangular mesh of the subdomain. Free triangular function 
has the flexibility to automatically control the number, size, and distribution of elements based on the available computing capability of the working computer. The total subdomain elements used in the simulation were set as 53,750 with the maximum element size of $8 \mu \mathrm{m}$. In order to get more accurate results at the bifurcation regions, resolution of curvature was adjusted to give a finer mesh along curved boundaries.

5. The laminar flow model and nonlinear solver were used in the convergence study.

6. After the convergence study, the velocity field in the pattern can be derived. Based on the velocity field, Reynolds numbers and the flow rate ratios can be obtained.

\subsection{Computational Results}

\subsubsection{Reynolds Number}

In fluid mechanics, the Reynolds number is a dimensionless number that gives a measurement of the ratio between inertial forces and viscous forces, and consequently quantifies the relative importance of these two forces in a given flow condition. The Reynolds numbers for microfluidics are typically very low, usually less than 1 , which means the flow is completely a laminar flow. We first calculated the Reynolds numbers of the main channel and six daughter channels, respectively. The results of Reynolds numbers in the daughter channels were much lower than that of the main channel as shown in Figure 5.2. The equation for the Reynolds number is 


$$
\operatorname{Re}=\frac{\rho v L}{\mu}
$$

In which, $\rho$ is the density of the fluid $\left(\mathrm{kg} / \mathrm{m}^{3}\right), v$ is the mean velocity, $L$ is a characteristic linear dimension, and $\mu$ is the dynamic viscosity of the fluid.

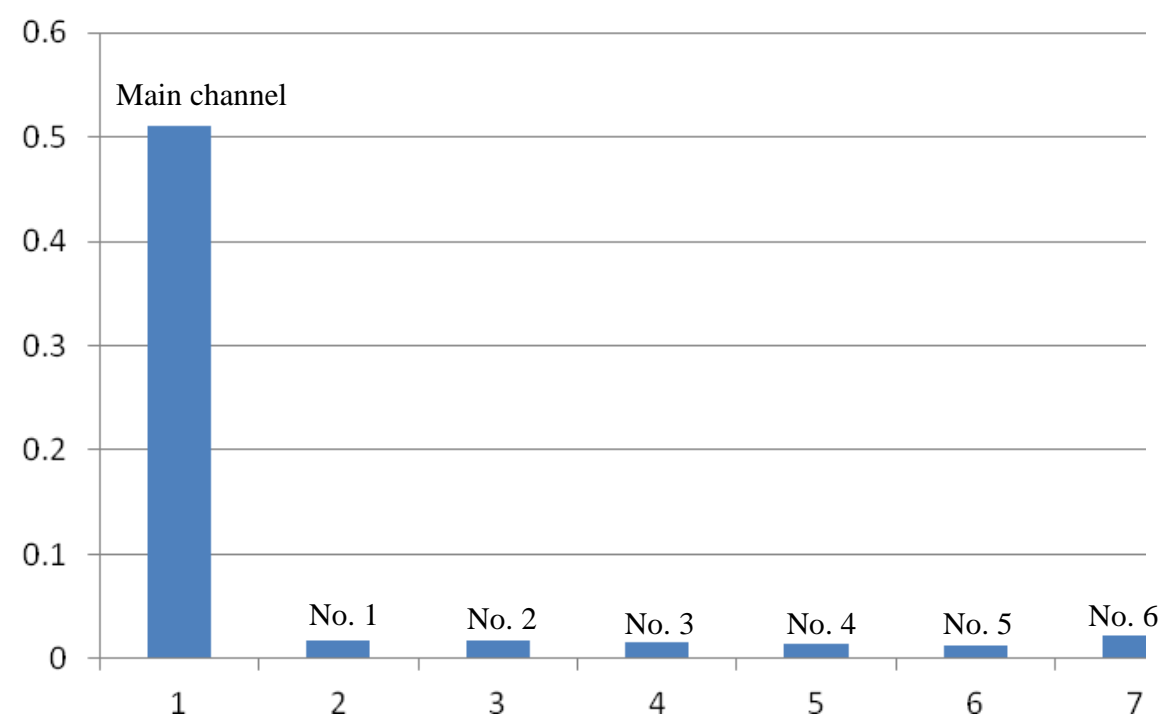

Figure 5.2 Reynolds numbers in the main channel and the daughter channels

As shown in the Figure 5.2, the first column represents Reynolds number in the main channel and it value was 0.51 . Columns from 2 to 7 represent Reynolds numbers for the six bifurcations, respectively, as indicated in the figure. These values were in the range from 0.01 to 0.02 . The Reynolds number in the main channel was one order magnitude larger than those of the daughter channels. The results quantitatively indicated the completely laminar flow in our device.

\subsubsection{Flow Velocity Profile}

Follow the steps in the previous section, we also ran the simulation to get the velocity field in the multiple bifurcation device. The simulation results were shown in Figure 5.3. Figure 5.3 (b) shows a close view of the first bifurcation. The velocity in the 
curvature channel was larger comparing with the velocity in the channel before the blood flows through the constriction channel.

a)

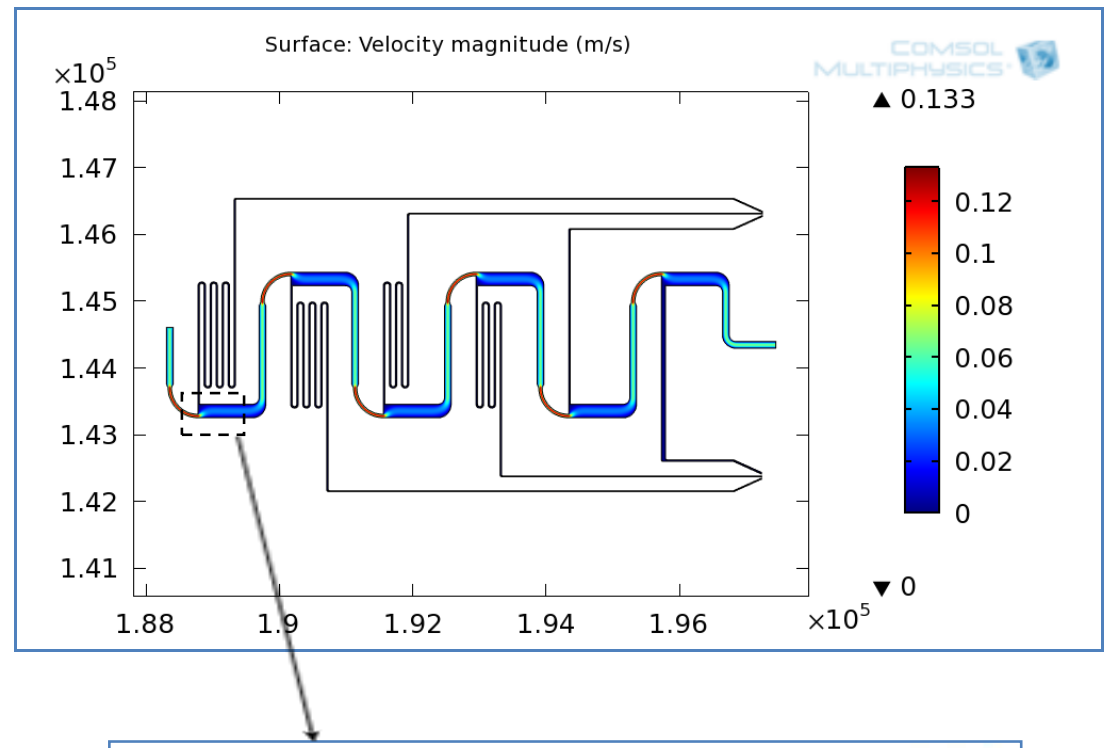

b)

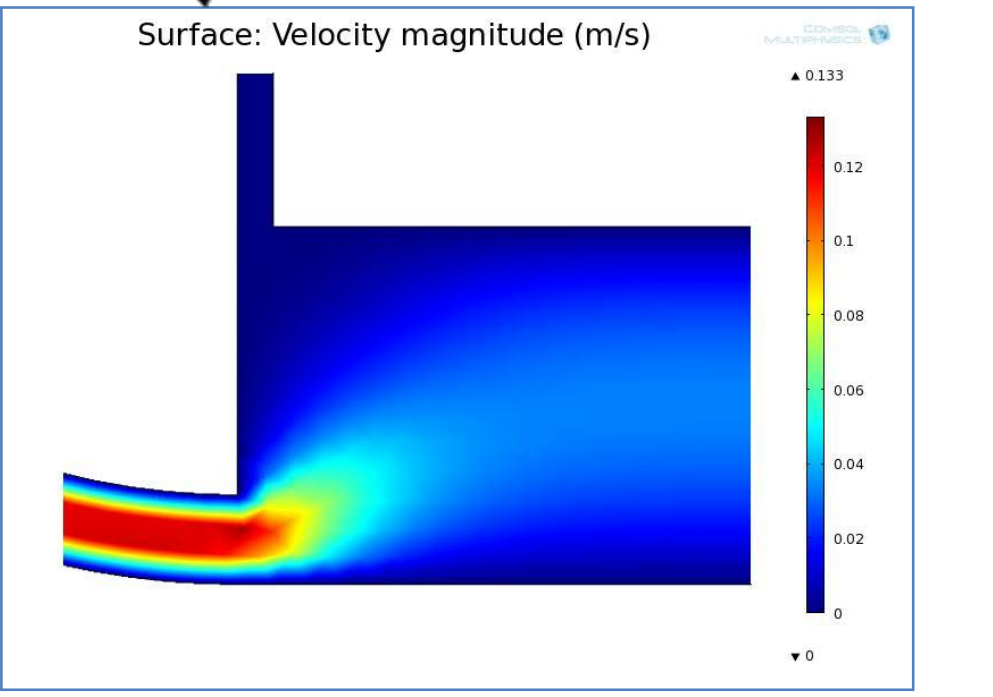

Figure 5.3 (a) Velocity filed of the multiple bifurcation device; (b) A detail view at the first bifurcation.

Because of the pressure driven flow and the no-slip wall conditions, the velocity profile was parabolic with the highest velocity at the center of the channel, and gradually decreased far from the center, which was the same as described in Xue's 
$\operatorname{paper}^{36}$.

\subsubsection{Velocity in the Daughter Channels}

Figure 5.4 shows the velocities at the daughter channels. The numbers in the y axis represented the bifurcation number and the numbers in the $\mathrm{x}$ axis represented the velocities.

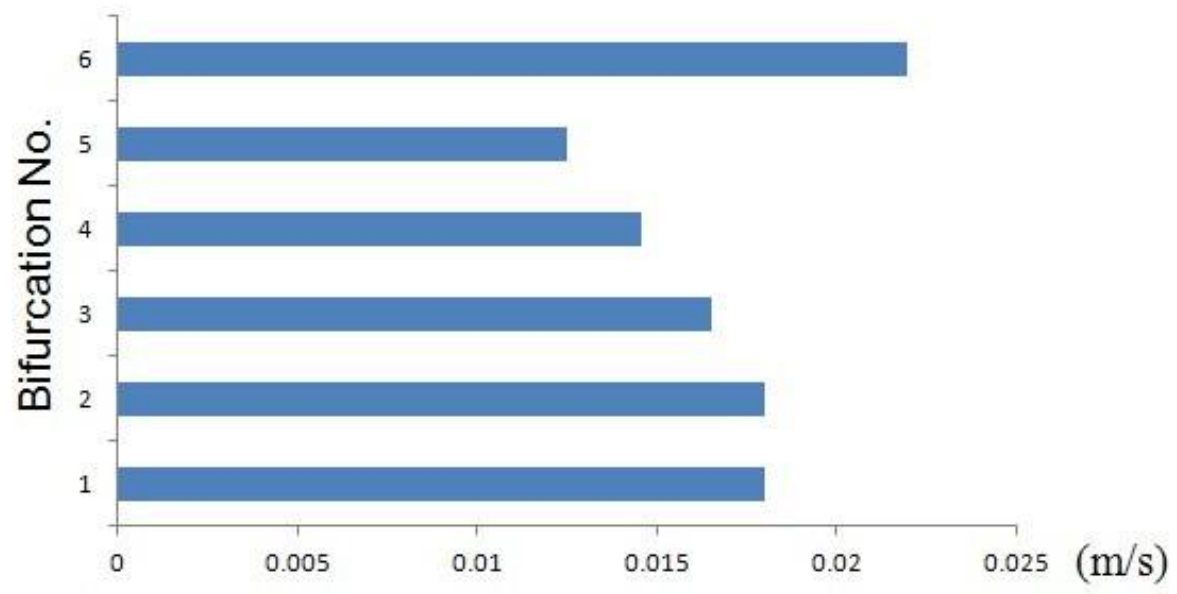

Figure 5.4 Flow velocities of the daughter channels

With the modifications of the channel dimensions, velocities can be kept at the same range. For the last $\left(6^{\text {th }}\right)$ bifurcation, because the length of the daughter channel was smaller than those of the others, the width of this channel was increased to keep the same flow rate ratio as other five bifurcations. Though in the same range, the velocity in the $6^{\text {th }}$ daughter channel was a little bit larger than the velocities in the other daughter channels as shown in the Figure 5.4.

\subsubsection{Flow Rate Ratios}

The flow rate ratios were obtained by taking the integrals of velocity profile of the daughter channels and the main channel, respectively, the flow rate at each channel 
was obtained, and then the flow rate ratio was calculated. Table 5.1 shows the flow rate ratio at each bifurcation.

Table 5.2 Flow rate ratio chart

\begin{tabular}{|c|c|}
\hline Bifurcation No. & Flow rate ratio \\
\hline 1 & $283.3: 1$ \\
\hline 2 & $281.9: 1$ \\
\hline 3 & $290.7: 1$ \\
\hline 4 & $334.5: 1$ \\
\hline 5 & $394.0: 1$ \\
\hline 6 & $247.2: 1$ \\
\hline
\end{tabular}

For the flow rate ratio calculation, because the heights were same for the daughter channels and the main channel, the flow rate of each channel was determined by the flow velocities, the channel width, and the channel length. By changing the widths of the daughter channels and the main channel, the channel resistances were modified. Thus, the flow rates were changed in each channel, which resulted in the changes of flow rate ratios as what we expected. The flow rate ratios were controlled to keep at the same range from 280:1 to $380: 1$ in the simulated design. From our experimental results in Chapter 6, we obtained the best separation with this design. According to the bifurcation law as discussed previously, if the flow rate ratios are large enough, the separation efficiency at each bifurcation could reach to $100 \%$. 


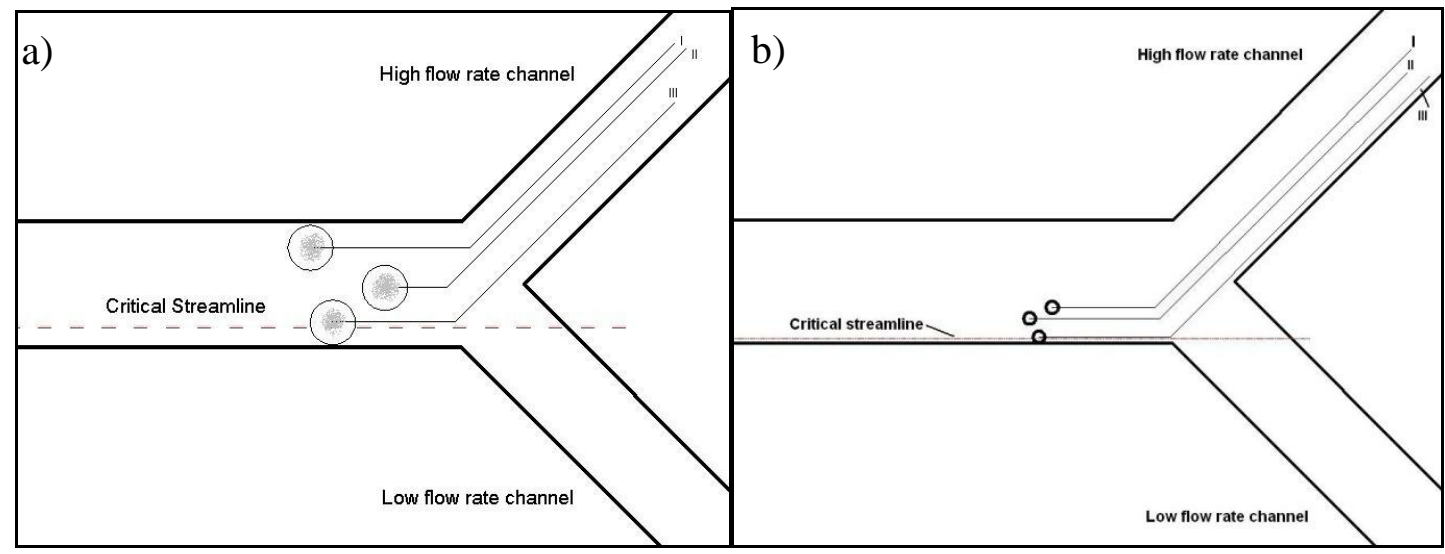

Figure 5.5 The critical streamline of (a) Cell-to-vessel diameter ratio is of the order of one; and (b) Cell-to-vessel diameter ratio is over the order of one.

Some other group has shown that the separation efficient could reach to $100 \%$ when the flow ratio was 6:1. In that case, the cell-to-vessel diameter ratio was of the order of one. $100 \%$ separation efficiency occurred when all the cells moved above the critical streamline (Figure 5.5 (a)). In our design, the diameter of main channel was $200 \mu \mathrm{m}$ and was over the order of one compared to the cell diameter (RBC: 6-8 $\mu \mathrm{m}$ ), thus the cell-to-vessel diameter ratio was much smaller. In order to keep $100 \%$ separation efficiency, the flow rate ratio at each bifurcation were increased and the critical streamline was close to the sidewall of the channel (Figure 5.5 (b)).

In summary, regarding to the bifurcation effects, a simplified model was developed and mainly used to examine the flow conditions (laminar flow, flow velocities, and flow rate ratio) in the designed the devices. However, we kept in mind that the bifurcation effects together with many other factors, such as the fluid properties (Non-Newtonian and Newtonian fluids), viscosity and its changes in different microchannels (Fåhræus-Lindqvist effect), the centrifuge forces generated by the 
curvature channel, affect the real experiment for the blood separation. A comprehensive model, however which beyonds the scope of this thesis research, is desired for considering all these factors together. In Chapter 6, we compared the centrifuge force effect in different designed channels, and showed that the centrifuge forces can assistant the plasma separation and increase the yield. 


\section{CHAPTER 6: Experimental Results of Multiple Bifurcation Device}

\subsection{Experimental Procedures}

The blood preparation followed the same procedures for single bifurcation channel. Whole blood with EDTA as an anticoagulant was diluted with $1 \mathrm{X}$ PBS to various hematocrit levels from $15 \%$ to $45 \%$. The tubing and microfluidic device were pre-wetted using $1 \%$ BSA solution for 1 hour at room temperature.

Similar to the operation procedures for single bifurcation device, the diluted blood was injected into the inlet and flew through the main channel and passed six bifurcations, respectively. A constriction/curvature changed the blood viscosity and exerted centrifuge forces on the cells to assist the separation as discussed previously. After flowing through these bifurcation regions, the plasma was separated into each daughter channel. The plasma from each daughter channel was merged together and outflew into the two reservoirs for collection. The separated blood cells will outflow into the central reservoir.

Fig. 6.1 (a) and Figure 6.1 (b) showed the schematic set-up and experimental set-up, respectively, including a syringe pump, a syringe with the diluted blood, the fabricated microfluidic device which was put under the Nikon microscope, and a computer with Nikon Element software for time-lapse imaging recording. At first, the blood was driven by the syringe pump into the microfluidic device through the 
connected tubing. The separated plasma flew down into the side plasma channels and collected inside of the reservoirs. In order to make sure the separated blood plasma was the same as the plasma separated by the conventional method, two groups of separated plasma were compared. One was from the multiple bifurcation device and the other was separated by centrifuge method (running at $5000 \mathrm{rpm}$ for 10 minutes). The two samples were sent to the clinical lab at WVU to examine and compare their protein components.

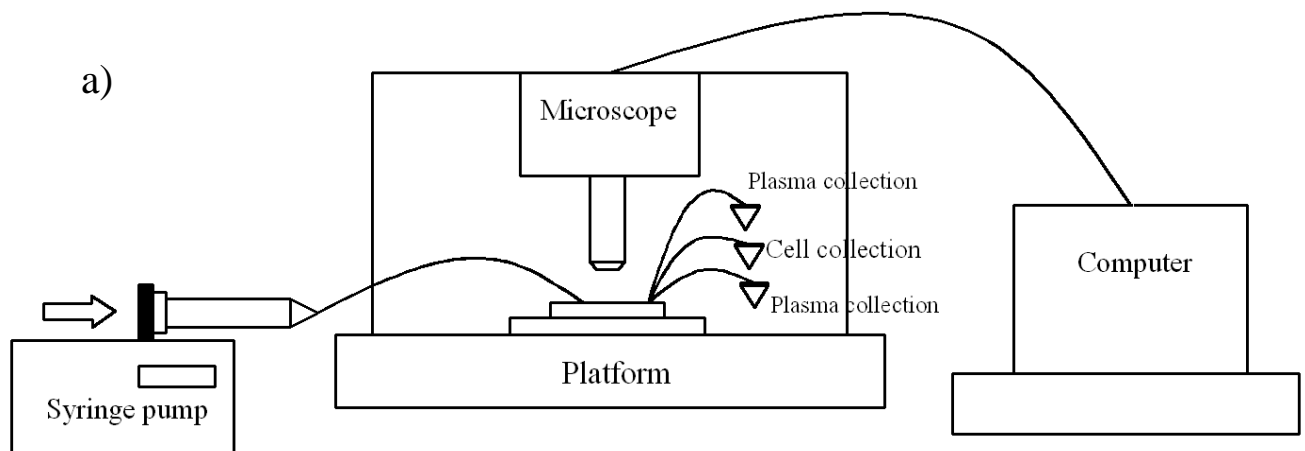

b)

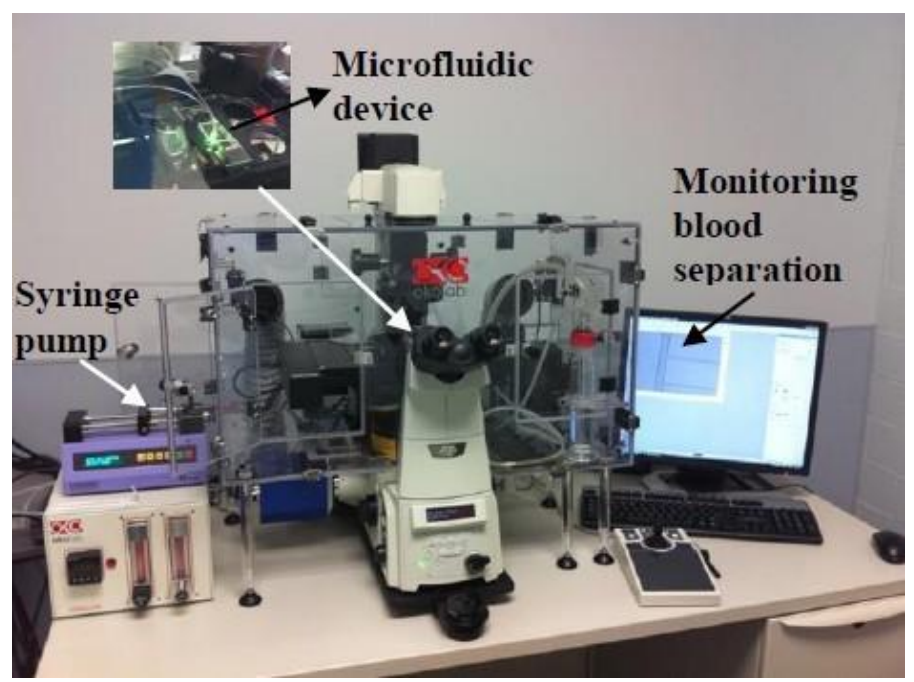

Figure 6.1 (a) The schematic of experimental setup. (b) Experimental setup for blood separation with real-time monitoring.

\subsection{Results}


The images were taken by the inverted microscope (Nikon Eclipse Ti, Japan) as shown in the Figure $6.2(\mathrm{a}, \mathrm{b}, \mathrm{c})$. These pictures showed that the blood separation at six bifurcations, respectively.

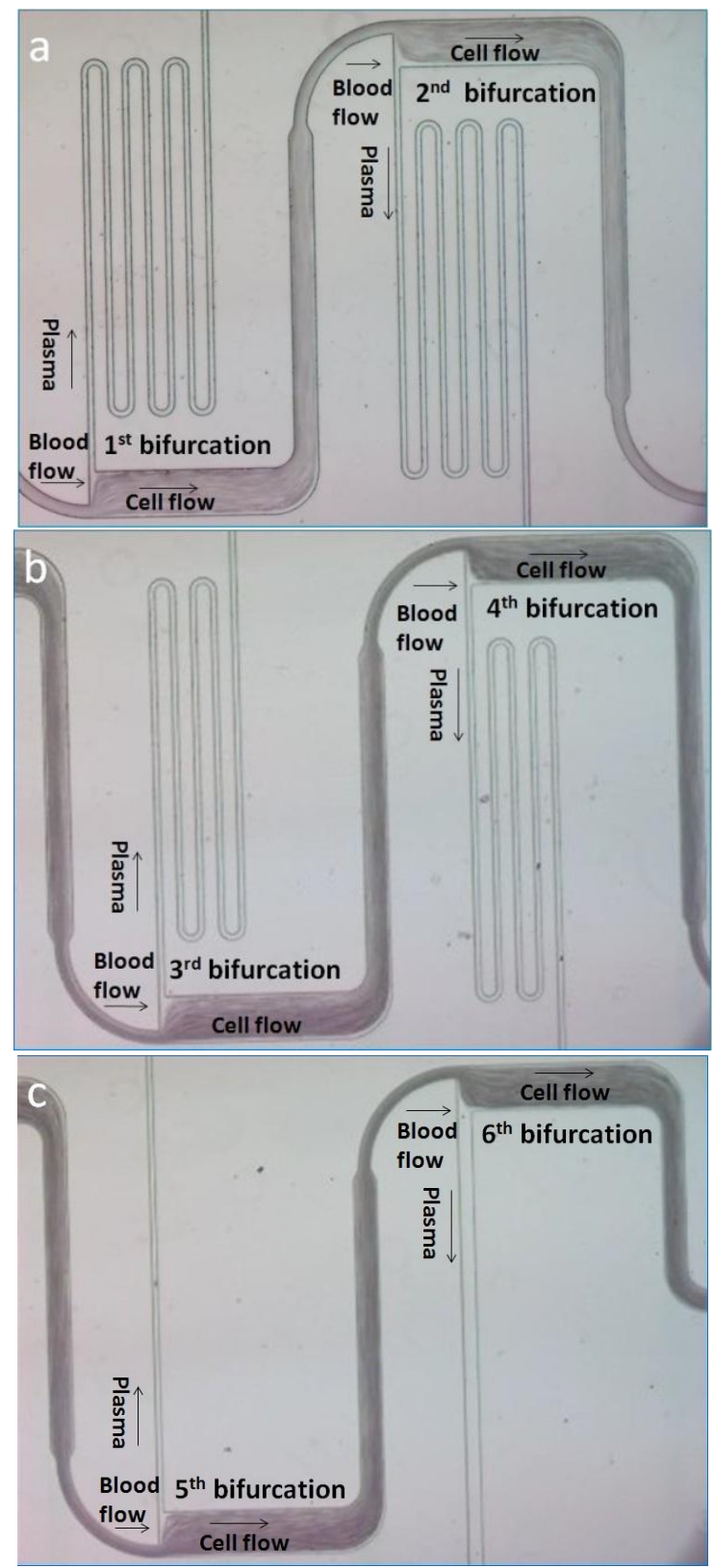

Figure 6.2 (a) Plasma separation at 1st and 2nd bifurcation. (b) Plasma separation at 3rd and 4th bifurcation. (c) Plasma separation at 5th and 6th bifurcation.

The red color shown in the pictures represents the blood cells, which were flowing in the main microchannel. Under the influence of centrifugation forces, the cells flew 
close to the outer edge of the main channel wall at the bifurcation region. The blood cells' trajectories were clearly observed and the cells were separated at each bifurcation region. Majority of the blood cells flew through the main channel and leave the cell free plasma flew into the daughter channels.

The flow rates of the blood were set from $5 \mu \mathrm{l} / \mathrm{min}$ to $25 \mu \mathrm{l} / \mathrm{min}$. We examined the flow rates' influence on the blood plasma separation efficiency. In addition, the blood samples with different hematocrit levels were varied from $15 \%$ to $45 \%$ to test the effects on the separation. The results showed that both running flow rates and hematocrit levels of the blood affect the separation efficiency and yield. We discussed this in more details in the following sections.

\subsection{Hydrodynamic Effects on Blood Separation}

As discussed in chapter 3, three hydrodynamic effects were applied in the device design. Because of the geometry difference of the main channel and the daughter channels (mainly the width of main channel was larger than the width of the daughter channels), the flow rate in the main channel was larger than the flow rate in the daughter channel, which was also validated by the COMSOL simulation. According to the bifurcation law, most cells flew through the main channel.

In the separation region, the curvature channel exerted a centrifuge force on the blood cells. The centrifuge force changes as the radius of the curvature channel changes. After the cells flew through this channel, they had the tendency to go through the 
outer edge of the main channel and leave the cell-free plasma flew into the daughter channels. The separation efficiently was increased with the modification of curvature channels.

Based on the Fåhræus-Lindqvist effect, the viscosities of the blood solutions in the main channel and curvature channels were different because of the different channel widths. The viscosity in the main channel was larger compared with the fluid viscosity in the curvature channel. However, because of the limited resource to measure the viscosity inside the microfluidic channel during the experiment, we have not been able to quantify the accurate viscosity effects on the separation.

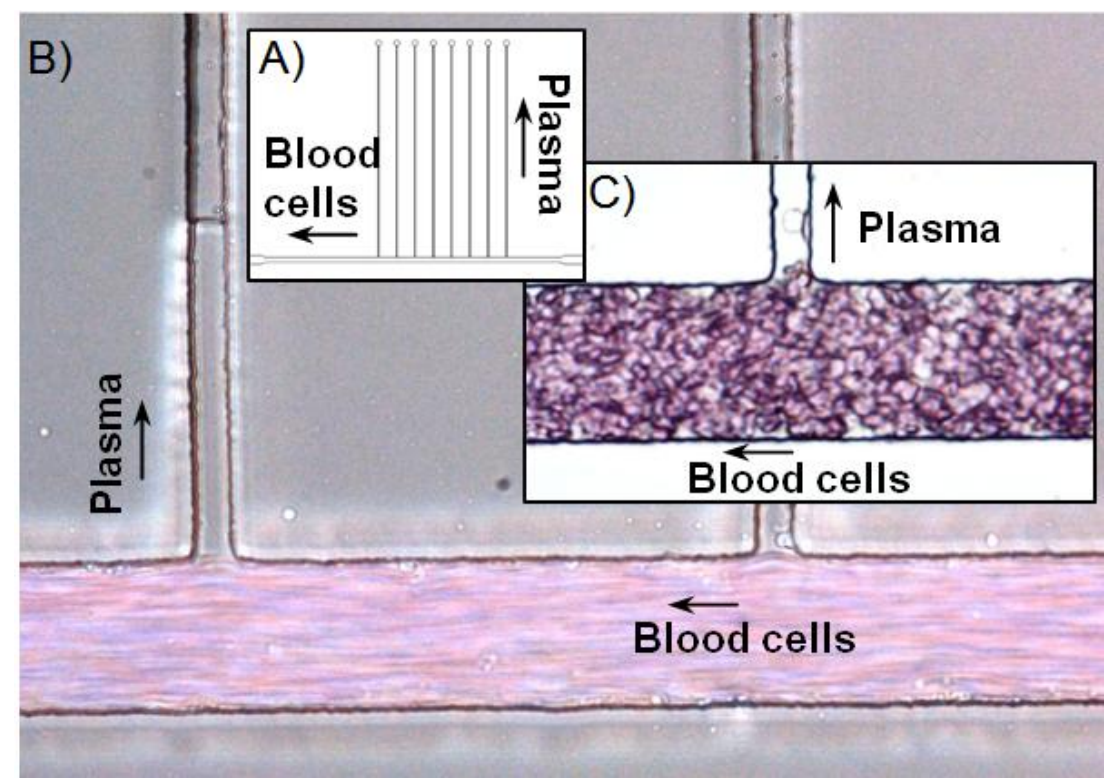

Figure 6.3 A) A microfluidic device. B) Blood separation in the microfluidic device. C) Higher magnification showing blood separation in the device.

We also tested a bifurcation microfluidic device without considering the centrifuge force. As shown in the Figure 6.3, the plasma was successfully separated through the bifurcation, however, the plasma yield was extremely low. The results helped to 
validate that the centrifuge forces generated by the curvature channels can assistant the plasma separation and increase the yield.

\subsection{Hematocrit Level Effect}

Figure 6.4 (a) shows the effects of the hematocrit levels on the separation efficiency and plasma yield. The plasma yield was defined as the separated plasma volume divided by the original plasma volume in the blood solution. The results showed that the yield of plasma separation was increased from $12 \%$ to $20.8 \%$ as the hematocrit level increased from $15 \%$ to $45 \%$ (whole blood).

a)

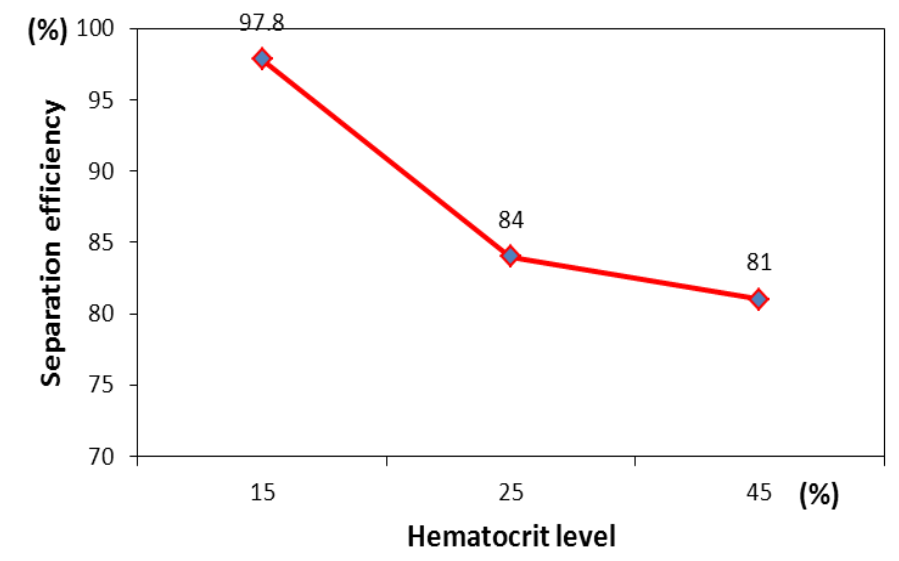

b)

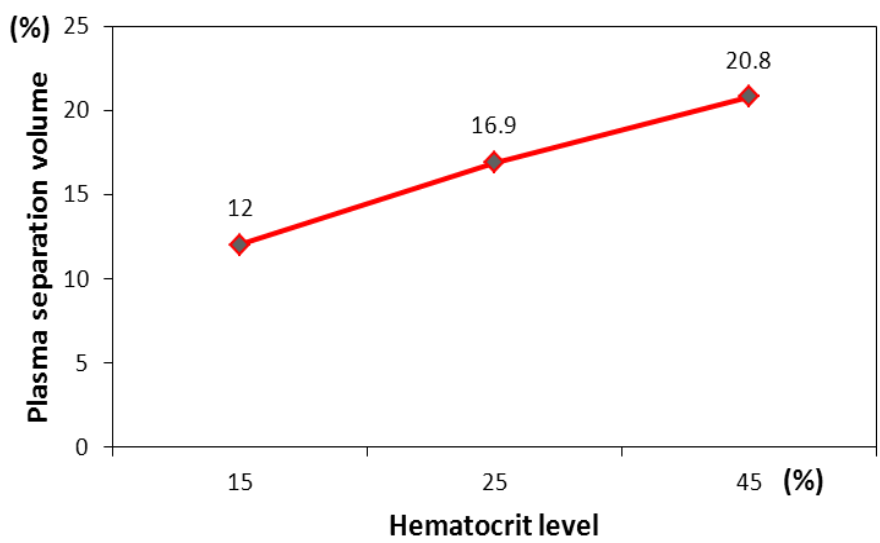

Figure 6.4 (a) Hematocrit level effects on the separation efficiency. (b) Hematocrit level effects on the plasma separation yield.

Ultra high speed CCD camera can been used to capture the blood cells in the 
microfluidic channels during their movement. Those images can clearly show the cells numbers in a channel, thus the separation efficient and yield can be accurately calculated. However, due to the lower speed of our microscopy camera, the separation efficiency in our experiment was estimated based on the solutions collected at the outlet reservoirs. The separation efficiency in our experiment was found to decrease from $98 \%$ to $81 \%$ with increasing hematocrit levels from $15 \%$ to $45 \%$ (whole blood) as shown in Figure 6.4 (b). Although increasing the hematocrit levels can reach a higher plasma yield, the separation efficiency was decreased.

The reason for decreased separation efficiency can be analyzed based on the hydrodynamic effects, such as the shear forces, centrifuge forces, and viscosity forces. As we discussed in the previous Chapter 3, different flow rates (different flow velocities) influenced the cells because the different shear forces were exerted on the cells' bodies. The asymmetric shear forces pull the cells into the high flow rate channels. A higher hematocrit level resulted in a larger density of cells and higher viscosities within the blood solution. A more pronounced viscosity forces existed in the higher hematocrit level solution that that in a lower hematocrit level solution diluted with PBS. Thus, for the low hematocrit level blood, the shear forces and centrifuge forces exerted on the cells were more easily overcome the relative lower viscosity forces and resulted in a better separation.

\subsection{Running Speed Effect}

The effect of different running speeds was studied as shown in Figure 6.5. For this 
testing, the hematocrit level was kept at the same level of $15 \%$. The running speeds were varied from $5 \mu \mathrm{l} / \mathrm{min}$ to $25 \mu \mathrm{l} / \mathrm{min}$ in the experiment.

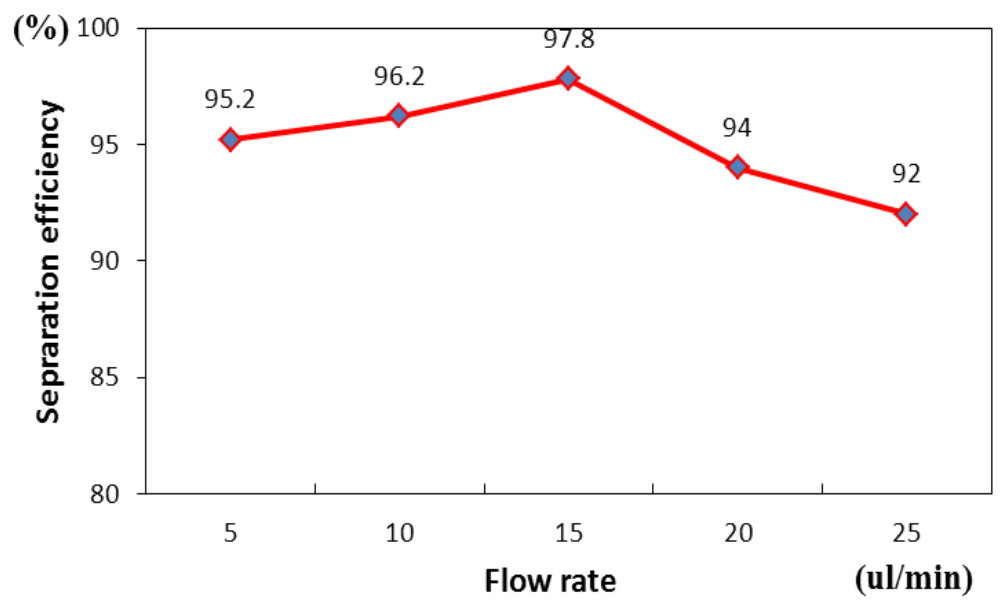

Figure 6.5 Separation efficiency at different running speeds when the hematocrit level was kept as $15 \%$.

As shown in this figure, the running speed at $15 \mu \mathrm{l} / \mathrm{min}$ showed the best separation efficiency as compared with other running speeds. Under lower flow rates, the shear forces exerted on the cells in the microchannel were not enough to drive the cells into the high flow rate channel, thus some of the cells flew into the plasma channel. Under higher running speeds, our results showed different phenomena compared with others. In Yang's paper, they found that the separation efficiency has the positive relationship with the running speeds ${ }^{28}$. More experiments should be performed to average the results for a solid conclusion.

\subsection{Electrophoresis Results}

In order to make sure that the microfluidic separated plasma has the same protein components as the plasma separated by conventional methods (centrifugation), we compared the plasma separated by both methods. The plasma as the control group 
(Figure 1.4) was extracted by the centrifuge method running at $5000 \mathrm{rpm}$ for 10 minutes. Figure 6.6 shows the separated plasma (test group) collected by the multiple bifurcation microfluidic device. The separation was done at the flow rate of $20 \mu \mathrm{l} / \mathrm{min}$ as the hematocrit level was kept at $15 \%$.

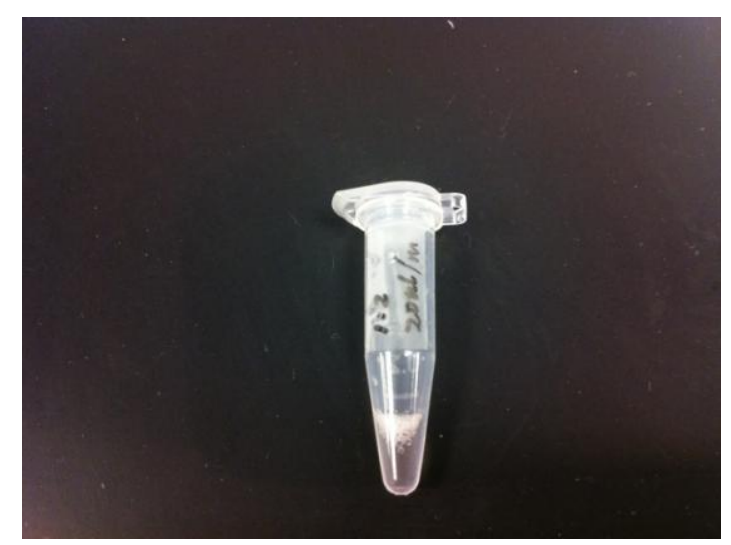

Figure 6.6 Microfluidic separated blood plasma (hematocrit level: 15\%)

The microfluidic separated plasma sample and centrifuged plasma sample were sent to WVU Clinical lab for the electrophoresis analysis. The electrophoresis analysis is an experimental process that blood plasma is placed on a special paper treated with agarose gel and exposed to an electric field to separate the proteins based on their sizes and electrical charges. Figure 6.7 (a) and (b) show the electrophoresis analysis based on the centrifuged plasma and microfluidic separated plasma.

As shown in figures, compared with the referece group, the plasma separated by our microfluidic device consist of major proteins in human blood plasma, including albumin, alpha 1, alpha 2, beta, fibrinogen (human plasma indicator), and gamm. The 
a)

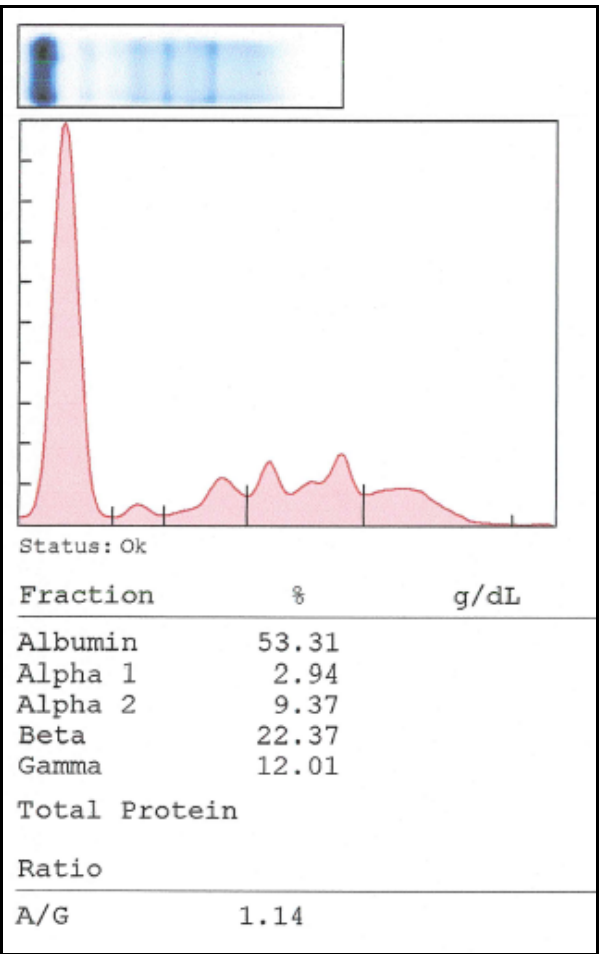

b)

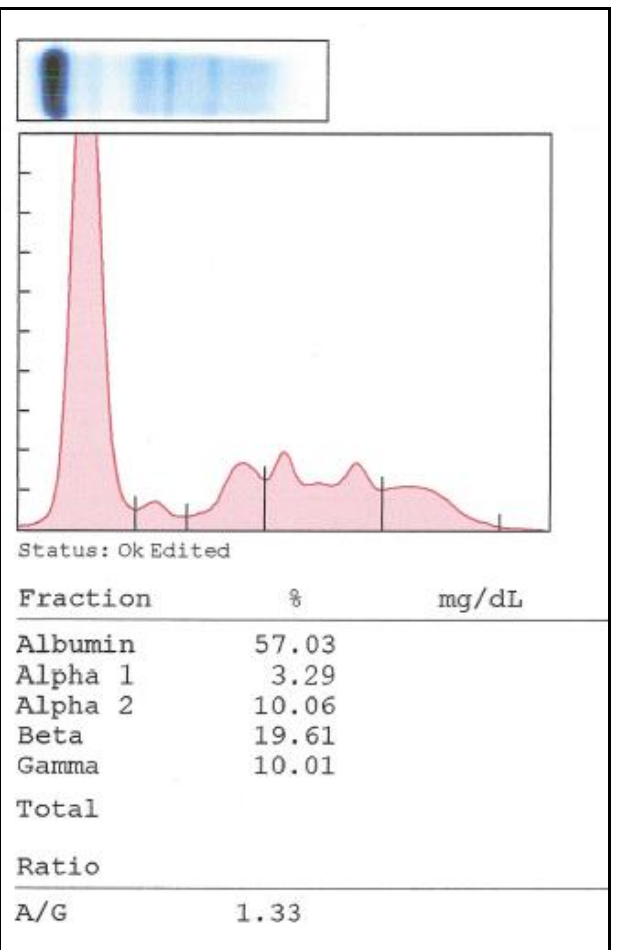

Figure 6.7 Electrophoresis analysis of (a) centrifuged plasma and (b) microfluidic separated plasma.

concentration of each protein were varified and shown to have a similar values as those presented in the control group. This demonstrates that the microfluidic separated plasma is almost the same as the plasma separated by the conventional method. 


\section{CHAPTER 7: Microfluidic Membrane Based Plasma Separation}

\subsection{Introduction}

There has been tremendous interest in the past decade using nanoporous or microporous membranes as functional units in biosensor development. These membrane-based technologies offer multiple advantages in sensing because of the nanoporosity or microporosity of the membranes that enables size-based trapping of biomolecules or particles. The membrane can be manufactured using standard processes with appropriate quality control for realizing some specifications, such as biocompatible and surface functions ${ }^{37}$.

Several approaches have been investigated for integration of the membrane within the microfluidic devices. Generally, it can be categorized into four methods: 1) Direct integration of commercial membranes; 2) Fabrication the membrane together with the chip fabrication process; 3) In-situ preparation of the membrane; and 4) Bulk materials functioned as a membrane ${ }^{38}$.

The first method is the most straight-forward, which is also the method used in our microfluidic membrane based device fabrication. This method simply uses clamping or gluing. Membrane can be easily prepared in-house in some cases, or directly purchased from a commercial supplier. Modifications can be applied to functionalize the membrane, such as by immobilization with trypsin ${ }^{39}$, bovine serum albumin 
$(\mathrm{BSA})^{40}$ or with an extraction fluid ${ }^{41}$. For our device, a commercial available membrane was used to trap blood cells from whole blood and extract the plasma.

Figure 7.1 shows the clamping method for fabricating the membrane which was embedded within the microfluidic device. The device consists of three layers with a membrane in between. The sealing between the membrane and the top and the bottom layers was achieved by using a glue.

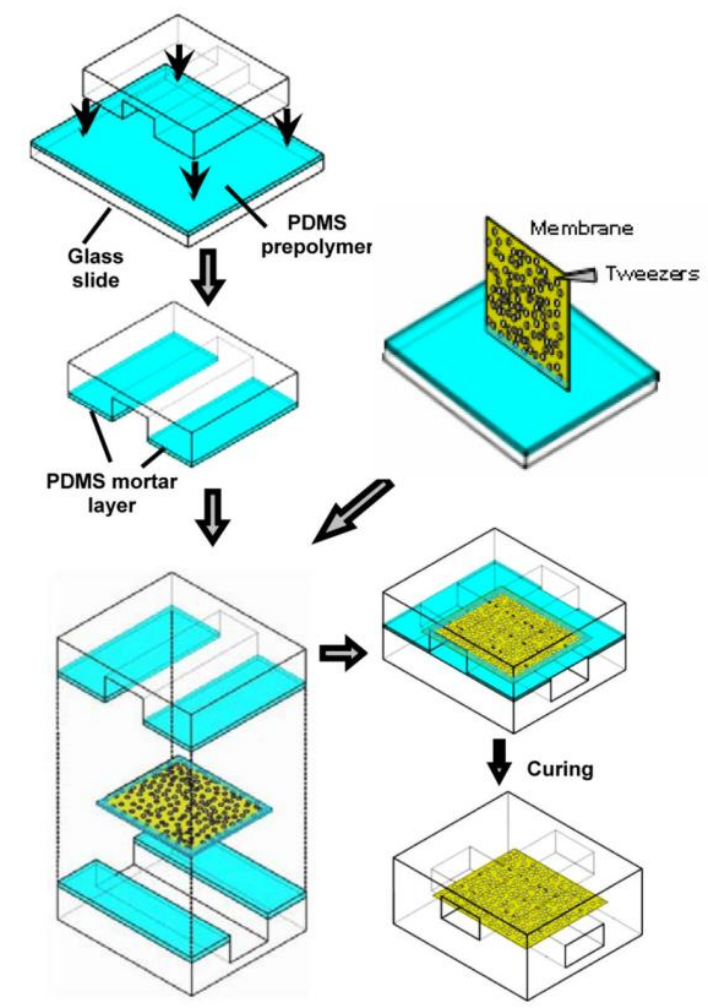

Figure 7.1 Clamping of the membrane between two PDMS layers ${ }^{42}$

The second approach is to prepare the membrane during the microfluidic device's fabrication. The process usually requires the sputtering and etching processes, which limit this method for being widely accepted because of the complicated fabrication process and high cost. 
The third method is in situ preparation of membrane. Moorthy and Beebe fabricated a porous membrane in the microfluidic channel by emulsion photo polymerization ${ }^{12}$. The last method is to choose a material that can be functioned as a membrane, such as PDMS. The advantages of PDMS have been discussed in Chapter 4. In Jong's paper, they used PDMS as the membrane ${ }^{43}$. Eight PDMS layers were built to integrate with an oxygen chamber. The permeability of PDMS is used to supply oxygen to cells. Water and carbon dioxide can also be exchanged through the membrane.
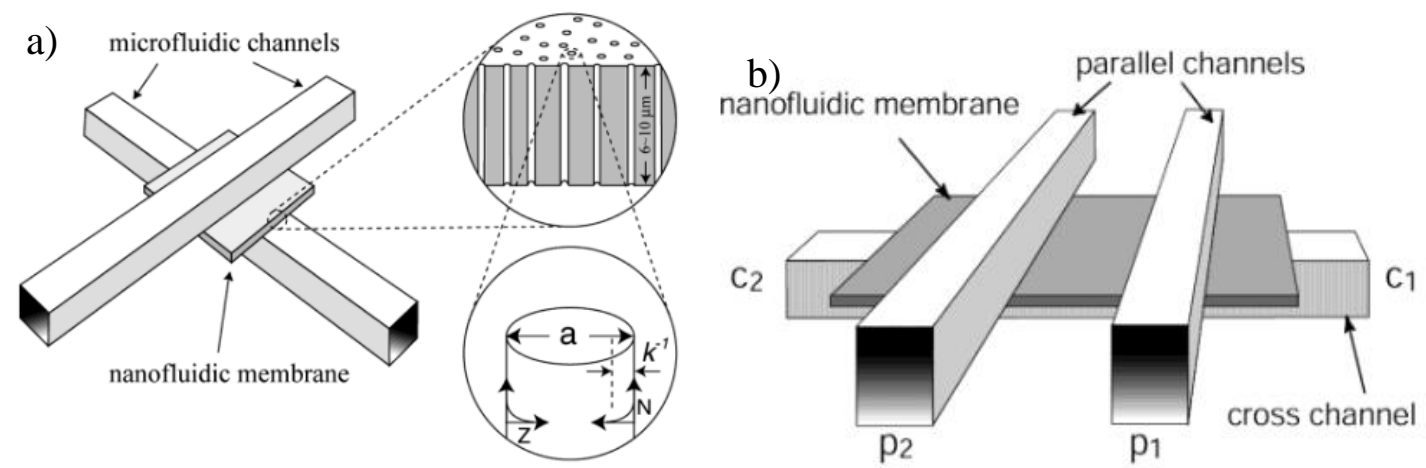

Figure 7.2 (a) Schematic design of crossed microfluidic channels separated by a nanometer-diameter capillary array interconnect. (b) Schematic design of a parallel channel system.

In Kuo's paper, they used nanofluidic membrane to build a microfluidic separation system $^{44}$. Figure 7.2 (a) shows the schematic design of this system, which was a crossed microfluidic separation system. The diameters of cylinder pores in the membrane were varied from $15 \mathrm{~nm}$ to $200 \mathrm{~nm}$. The authors also developed a parallel channel system as shown in Figure 7.2 (b). Figure 7.3 shows the picture of the microfluidic separation device. The white rectangle shown in the device was the polycarbonate track-etched (PCTE) membrane (200 nm diameter pores). 


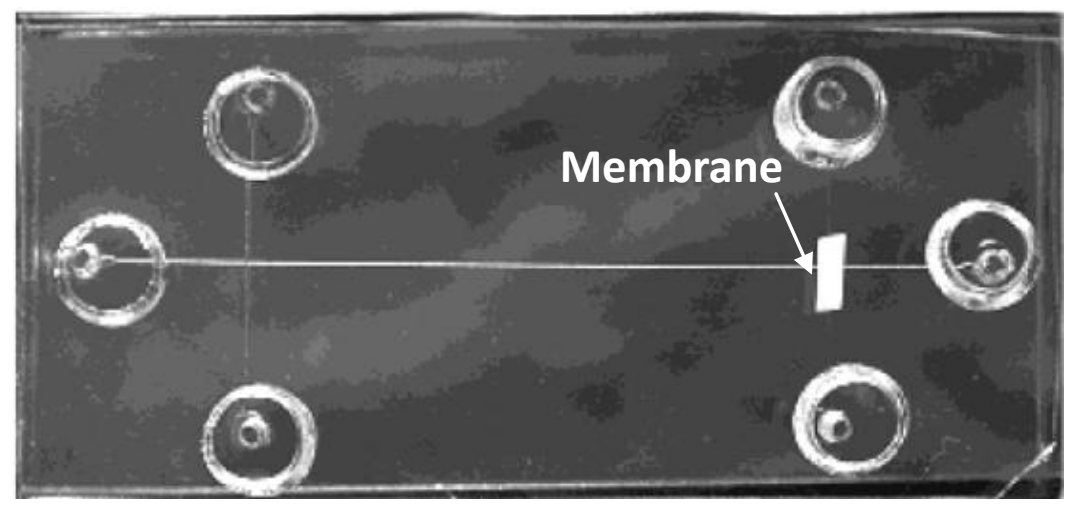

Figure 7.3 Photograph of a microchip with crossed microchannels and a nanofluidic membrane

\subsection{Vivid Plasma Separation Membrane}

For our microfluidic device we used Vivid Plasma separation membrane from Pall Life Science Corporation. It is a highly efficient membrane for one-step plasma separation from whole blood without the use of centrifugation. The patented Vivid Plasma Separation membrane is specifically engineered and optimized for the generation of high quality plasma for use in downstream diagnostic assays. Constructed of asymmetric polysulfone, the membrane efficiently captures the cellular components of whole blood resulting in plasma that contains minimal cellular contaminants. The asymmetric structure of the membrane gently captures the cellular components without lysis which is a contrast from glass fiber media that often shears and lyses cells leading to contamination of the plasma ${ }^{45}$.

In summary, the membrane can 1) Achieve high quality plasma in less than two minutes with $\geqslant 80 \%$ plasma yield; 2) Hemolysis levels significantly lower than 
glass fiber media generated plasma; 3) Low, non-specific binding of common diagnostic biomarkers and target analytes; 4) Efficient removal of the cellular components of whole blood, including red cells, white cells, and platelets.

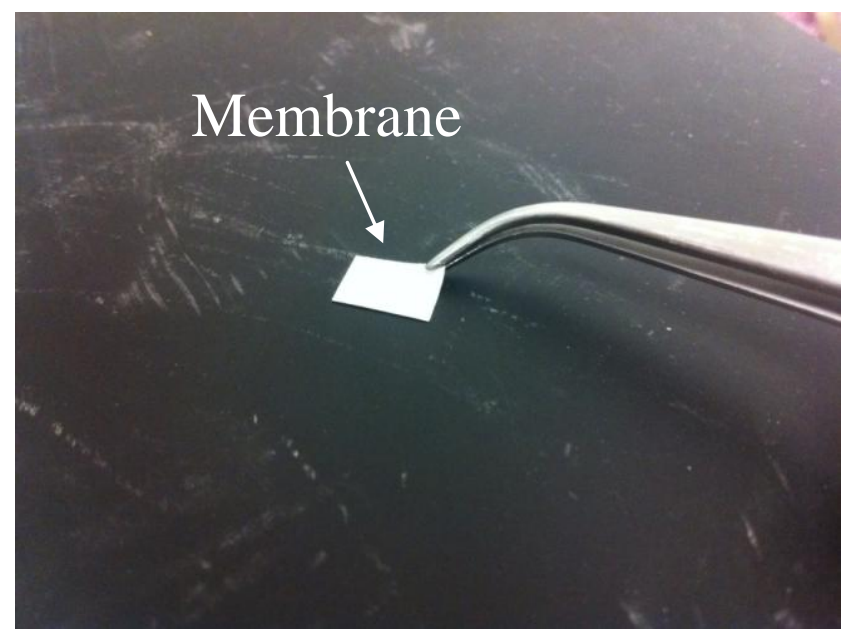

Figure 7.4 Vivid plasma separation membrane

They provide the membrane with three grades: GF, GX and GR, which have different membrane cell trapping capacities for obtaining different plasma yields. The thickness of membrane is around $330 \mu \mathrm{m}$. The yield of separated plasma depends on the area of membrane. For example, the recommended whole blood sample volume for GF grade membrane is $20 \mu \mathrm{l} / \mathrm{cm}^{2}$. Based on the yield $\geqslant 80 \%$, at least about $8 \mu \mathrm{l}$ plasma can be extracted for this grade membrane $\left(1 \mathrm{~cm}^{2}\right)$ from whole blood ( $45 \%$ hematocrit level).

Figure 7.5 shows the blood cells flow through the membrane and demonstrated the filtration ability of Vivid plasma separation membrane. The left side picture show the picture of a clean membrane. The right side picture shows the blood flowing through the membrane, and the red blood cells were trapped in the pores of membrane. The 
upper layer was filled with the blood cells and the lower layer was the separated cell-free plasma.

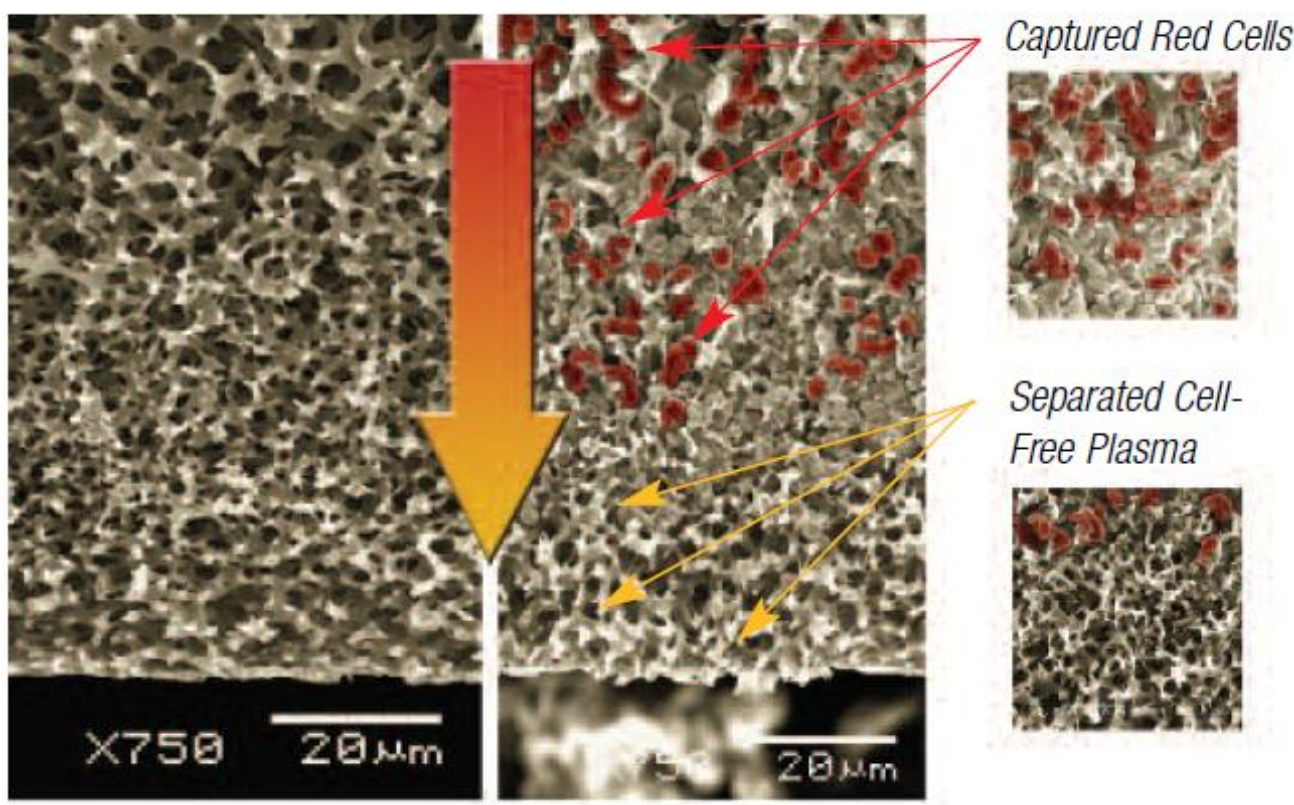

Figure 7.5 High quality plasma generation using vivid plasma membrane ${ }^{45}$

\subsection{The Darcy's Law}

The Darcy's law describes the flow of a fluid through a porous medium. It is simple proportional relationship between the instantaneous discharge rate through a porous medium, the viscosity of the fluid and the pressure drop over a given distance ${ }^{46}$.

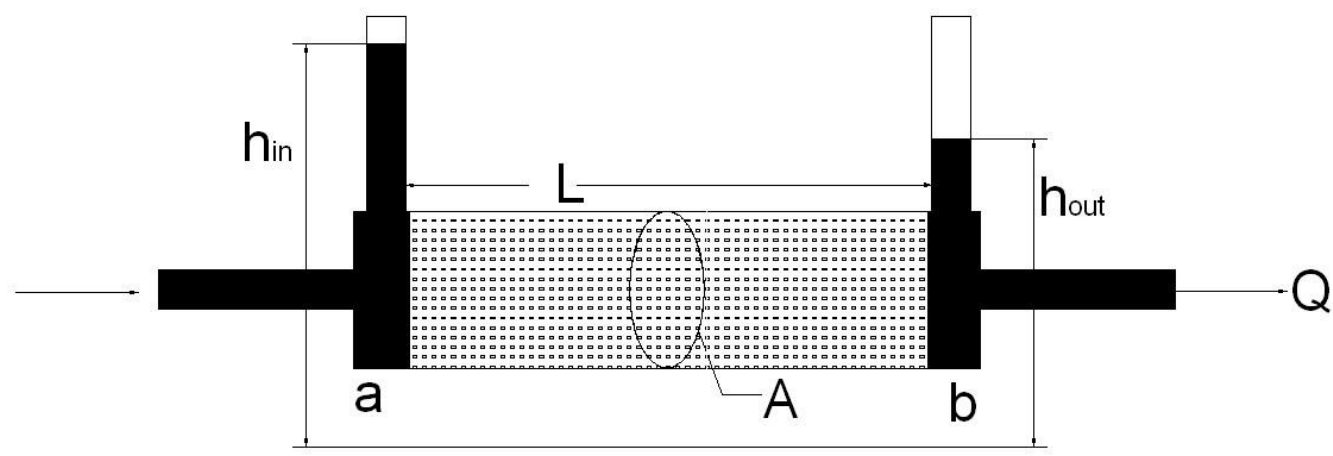

Figure 7.6 Working mechanism of Darcy's law in two dimensions

Figure 7.6 shows the working mechanism of Darcy's law in two dimensions. Q is the 
total discharge (Volumetric flow rate). The relationship between these parameters is as the follows.

$$
\mathrm{Q}=-\mathrm{kA} \frac{\Delta h}{L}
$$

A: The cross sectional area of flow; $\Delta h$ : Hydraulic head representing the pressure difference. $\Delta h=\mathrm{h}_{\text {out }}-\mathrm{h}_{\text {in }} ; \mathrm{k}$ : Hydraulic conductivity. From Darcy's law, the volume of separated plasma can be derived that it is proportional to the area of the membrane.

\subsection{Device Fabrication}

As mentioned above, the glue-bonding (liquid PDMS bonding) method was used to integrate the membrane into the microfluidic device. However, the direct bonding of the membrane and the device was challenging because of the thickness of the membrane $(330 \mu \mathrm{m})$, which prevented a completely sealing. The solution (Figure 7.7) was to use a 3 inch silicon wafer $(350 \mu \mathrm{m})$, which has the similar thickness as the membrane. First, a small piece of 3 inch silicon wafer was cut and it was used as the mold for PDMS replication into the reservoir. The dimensions of the reservoir were similar to the size of the trimmed membrane. Then we used the soft lithography to get a PDMS layer 1 with this reservoir. On the other side, the PDMS layer 1 was cut with a rectangle hole which was a little smaller than the reservoir created from the silicon wafer mold in order to have some edges to bond the membrane. Liquid PDMS was applied to the edges of the membrane on both sides. The membrane was put inside the reservoir, then a PDMS layer 2 was bonded with membrane and the PDMS layer 1. 


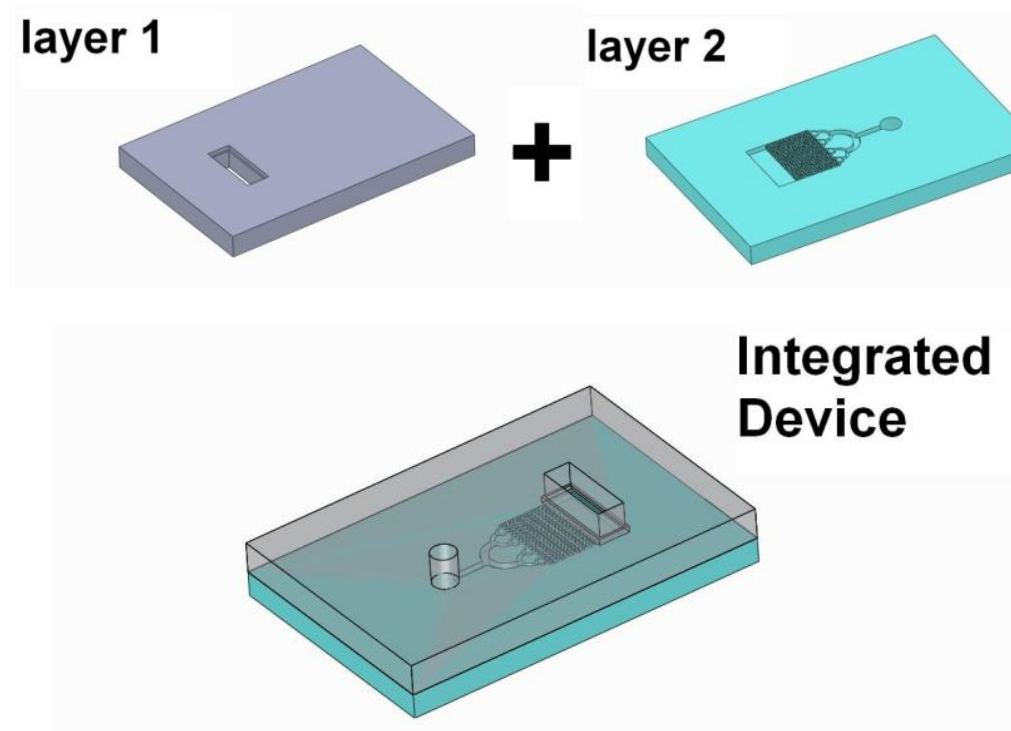

Figure 7.7 Schematic fabrication process of the microfluidic membrane based device

In addition, the PDMS layer 2 consisted of a channel with small post arrays to generate capillary forces to extract and delivery the separated plasma from the membrane. Figure 7.8 shows the details of the device pattern. The liquid driven by the capillary forces can flow through these narrow spaces without any other external forces. It occurs because of the inter-molecular attractive forces between the liquid and solid surrounding surfaces ${ }^{47}$. With this pattern design, the separated plasma can be extracted into the small feature array microfluidic channels.

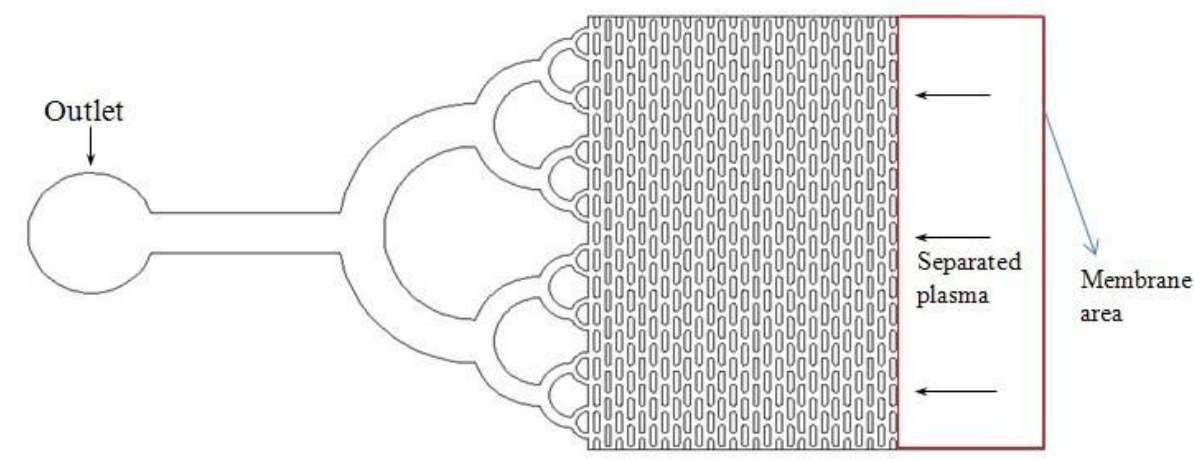

Figure 7.8 Microfluidic device pattern of membrane based blood plasma separation 


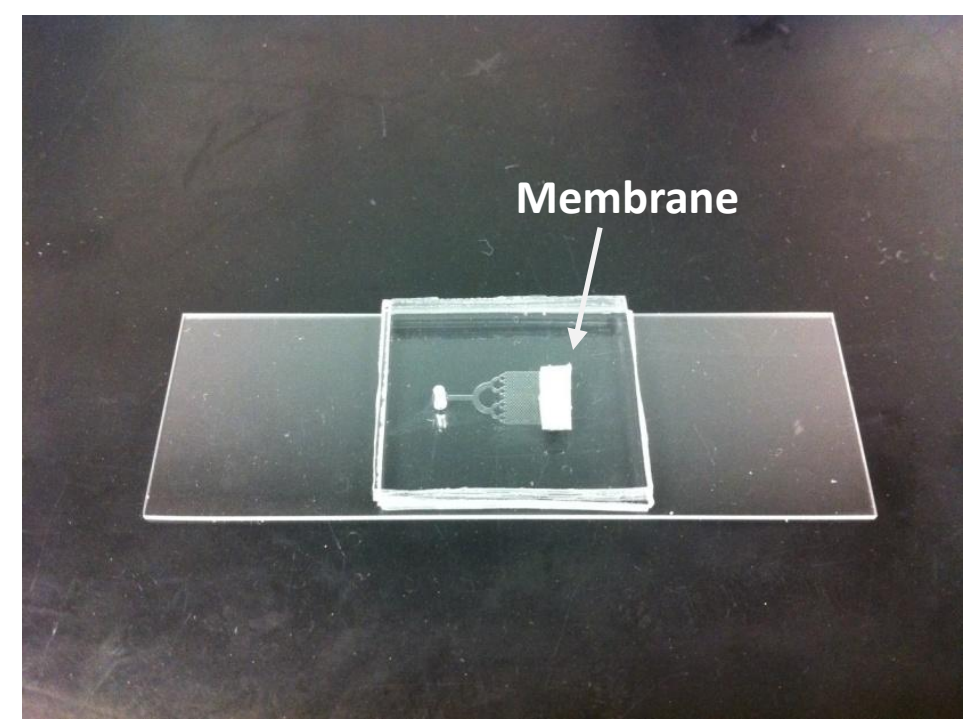

Figure 7.9 The membrane based device

\subsection{Experimental Results}

Before the experiment, the device was put in a oxygen plasma cleaner for oxygen plasma treatment for 90 seconds. Then the diluted blood was loaded into the inlet. The device was put under the microscope for observation. Figure 7.10 shows the blood separation using the membrane based device. The dark area in right of the figure was the membrane area. The separated plasma flew from the right to the left, and the separated plasma can continuously flow through the post array channel to the outlet.

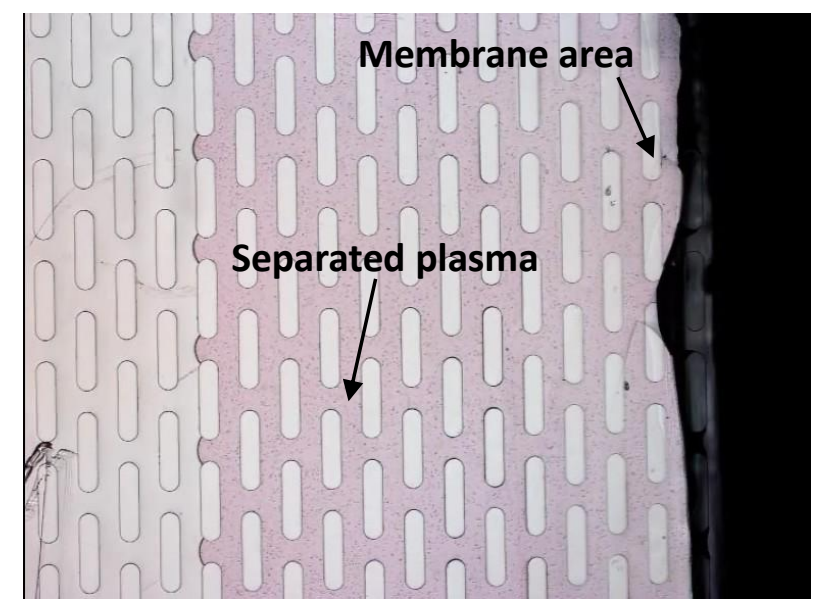

Figure 7.10 The picture shows the plasma separation using a membrane based device 


\subsection{Conclusions}

A double layer membrane based blood plasma separation device has been successfully fabricated. Membrane was directly incorporated in the microfluidic device, and the capillary microfluidic pump was able to extract and delivery the separated plasma from the membrane.

The device has several advantages. It does not require an external power and is a passive plasma separation method. Capillary force and gravity force drive the blood through the membrane and separate the plasma. The separation efficiency and yield are determined by the properties of the membrane. However, we found that the blood conditions affect the separation efficiency and plasma yield. The freshest extracted blood should be used for the experiment because the blood cells tend to lysis after the extraction, and a few days old blood can affect the quality of the plasma separation. In addition, the surface treatment of the PDMS device showed some effects to the plasma separation. We will further investigate this issue to exam the plasma based on their separation rate and efficiency. Finally, because the yield of the plasma separation is determined by the membrane grades and sizes, the volume of the plasma is necessary to be calculated based on the membrane dimensions and application needs. 


\section{CHAPTER 8: Conclusions and Future Work}

\subsection{Conclusions}

In this thesis work, we developed both active and passive microfluidic based human blood plasma separation devices. In Chapter 3, 4, 5 and 6, we discussed the hydrodynamic effects for microfluidic plasma separation and special channel designs. Then COMSOL simulation was applied for the optimization of the design and assistance to verify the design. In chapter 7 , we developed the microfluidic membrane based device for blood plasma separation. The following conclusions were drawn from the results of this research:

1. Both active and passive microfluidic blood plasma separation devices were successfully fabricated.

2. Several hydrodynamic effects were applied in the microfluidic continuous plasma separation, which are bifurcation law, centrifuge force and Fåhræus-Lindqvist effect.

3. COMSOL simulation is convenient for us to optimize the design.

4. Hematocrit levels affect the separation efficiency and plasma yield.

5. The plasma separation rate of the device can reach up to $98 \%$ and plasma yield can reach to $20 \%$.

6. The electrophoresis results showed that the microfluidic separated plasma has the same protein components as the plasma separated by centrifugation. 
7. Small blood handling, high plasma yield and separation efficiency can be obtained by the continuous active microfluidic blood plasma separation techniques.

8. The microporosity of the Vivid membrane efficiently enables the trapping of blood cells from the whole blood. The size of the membrane determines the plasma yield.

9. Small blood handling and extra power free are the benefits for the passive membrane based microfluidic plasma separation.

\subsection{Future Work}

We have developed the microfluidic devices for separating the plasma from the whole human blood. In addition, we are developing PDMS on-chip microvalve technologies in our lab and are working to integrate the microfluidic plasma separation unit with a multiplex heavy metal sensor into a Lab-on-a-Chip (LOC) device. LOC is a technique to perform chip-scale assays driven by microfluidics to automate and 'streamline' different chemical or biological processes (Figure 8.1).

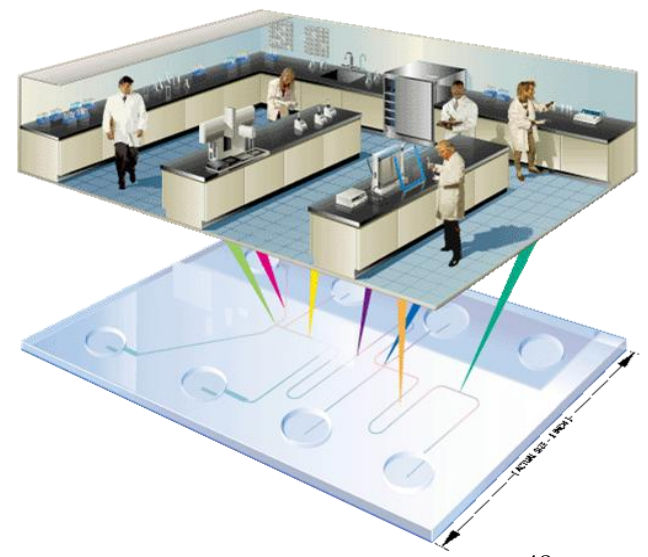

Figure 8.1 Lab on a Chip ${ }^{48}$

The applications of LOCs include many aspects. In Chin's paper, they investigated the 
past studies and future opportunities of LOCs for the global health ${ }^{49}$. In their review, they discussed communicable and non-communicable diseases, such as HIV/AIDS, malaria, measles, cancer, respiratory diseases, and diabetes mellitus. These diseases would expect to be detected by LOCs in the near future, and some LOCs have been available for simple operation and rapid detection.

In Figeys's paper, they revealed the revolution of LOCs in biological and medical sciences ${ }^{50}$. They pointed out that the miniaturization of devices can efficiently reduce the cost for device fabrication and reagents consumption. In human genome project, LOCs could integrate with excitation source, pressure source or control circuitry for identifying DNA. In the future, novel materials and fabrication processes will likely be used for next generation of microfabricated devices.
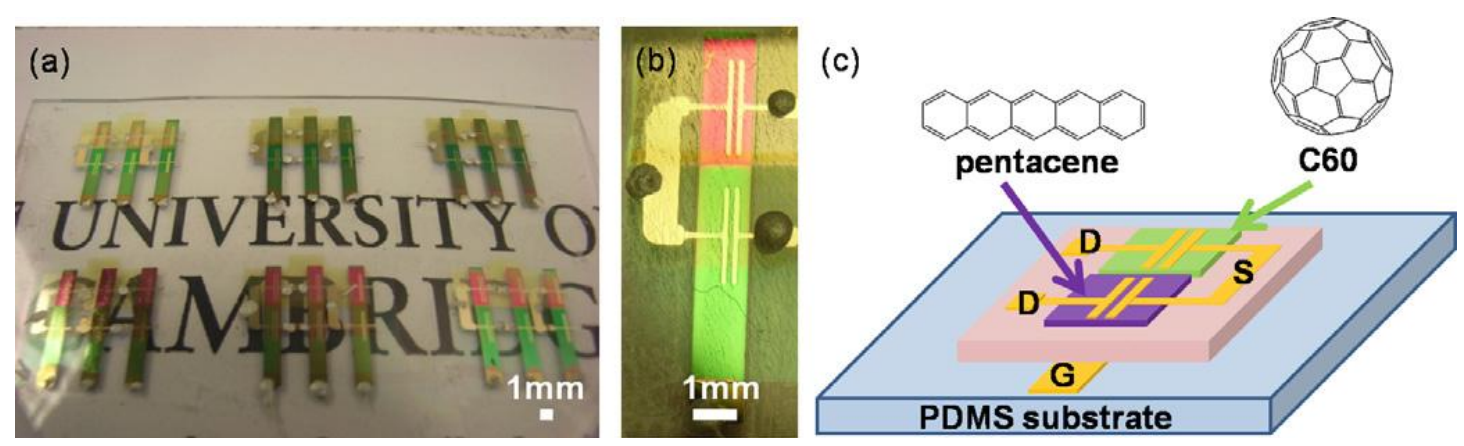

Figure 8.2 (a) Photograph of an array of complementary organic inverters on $1 \mathrm{~mm}$ thick transparent PDMS. (b) The detailed view of inverter device. (c) Schematic design of the complementary inverter stack directly prepared on PDMS.

LOCs are now extending their abilities to new areas. In Graz's paper, they built an organic circuit on a new class of substrate, silicone elastomers ${ }^{51}$. Figure 8.2 show the photograph and schematic of this device. This device demonstrated the feasibility of 
organic electronics on plastic. In the future, many these electronic LOCs will be created for a variety of applications.

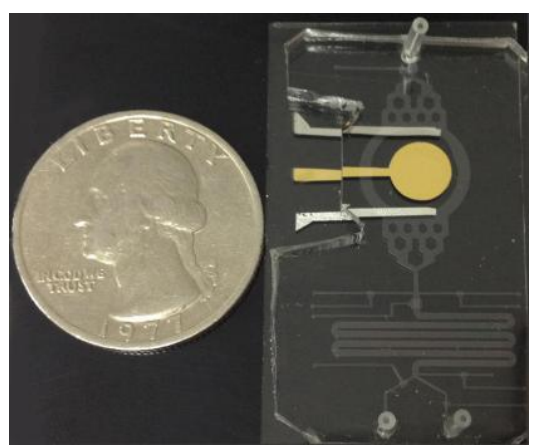

Figure 8.3 An integrated microfluidic electrochemical sensor based on planar electrodes.

A microfluidic LOC electrochemical sensor platform has been demonstrated by our group and this work is collaborated with Dr. Wu's group at WVU. (Figure 8.3) The figure shows the LOC includes a microfluidic mixer and the sensor electrodes. The microfluidic blood separation unit will be integrated with the LOC. This LOC device could expect to offer many advantages, such as small blood volume handling, fast and effective detection, and portable for point-of-care applications. 


\section{REFERENCES}

1 J. Witnesses. Position Overview. Bloodless Medicine \& Surgery, 2007. <http://www.pennmedicine.org/health_info/bloodless/000206.html>

2 M. Toner, D. Irimia. Blood-on-a-Chip. Annual Review Biomedical Engineering, 7, 77-103, 2005.

${ }^{3}$ Extreme Health USA Corporation. Kelation Therapy. <http://www.kelation-therapy.com/>

${ }^{4}$ M. Kaloyianni, S Dailianis, E. Chrisikopoulou, A. Zannous, S. Koutsogiannaki, D. Alamdari, G. Koiakos, V. Dimitriadis. Oxidative Effects of Inorganic and Organic Contaminants on Haemolymph of Mussels. Comparative Biochemistry and Physiology. 149, 631-639, 2009.

5 A. Sallam, N. Hwang. Human Red Blood Cell Hemolysis in a Turbulent Shear-flow: Contribution of Reynolds Shear Stresses. Biorheology, 21, 783-797, 1984.

${ }^{6}$ M. Kersaudy-Kerhoas, D. Kavanagh, R. Dhariwal, C. Campbell, M. Desmulliez. Validation of a Blood Plasma Separation System by Biomarker Detection. Lab Chip, 10, 1587-1595, 2010.

${ }^{7}$ E. Stern, A. Vacic, N. K. Rajan, J.M. Criscione, J. Park, B. R. Ilic, D. J. Mooney, M. A. Reed, T. M. Fahmy, Label-free biomarker detection from whole blood. Nature Nanotechnology, 5, 138-141, 2010.

${ }^{8}$ W. Yang. New Biomedical Microdevice Detects Cancer Faster \& With Less Pain. $<\mathrm{http} / / /$ therohanaurora.com/new-biomedical-microdevice-detects-cancer-faster-with-1 ess-pain/>

${ }^{9}$ H. Shapiro. Practical Flow Cytometry. New Jersey: John Wiley \& Sons, Inc., fourth edition, 2005.

10 Abcam Corporation. Flow Cytometry Guide.

$<$ http://docs.abcam.com/pdf/protocols/Introduction_to_flow_cytometry_May-10.pdf

$>$

11 L. Terstappen, S. Johnsen, M. Segers-Nolten, M. Loken. Identification and Characterization of Plasma Cells in Normal Human Bone Marrow by High-Resolution Flow Cytometry. Blood, 76, 1739-1747, 1990.

12 J. Moorthy, D. J. Beebe. In Situ Fabricated Filters for Microsystems. Lab Chip, 3, 62-66, 2003.

13 J. Zoval, M. Madou. Centrifuge Based Fluidic Platforms. Proceedings of the IEEE, 
92, 776-781, 2006.

${ }^{14}$ S. Haeberle, T. Brenner, R. Zengerle, J. Ducree. Centrifugal Extraction of Plasma from Whole Blood on a Rotating Disk. Lab Chip, 6, 776-781, 2006.

15 N. Korin, A. Bransky, U. Dinnar. Theoretical Model and Experimental Study of Red Blood Cell (RBC) Deformation in Microchannels. Journal of Biomechanics, 40, 2088-2095, 2007.

${ }^{16}$ P. Wilding, L. Kricka, J. Cheng, G. Hvichia, M. Shoffner, P. Fortina. Integrated Cell Isolation and Polymerase Chain Reaction Analysis Using Silicon Microfilter Chambers. Analytical Biochemistry, 257, 95-100, 1998.

17 L. Huang, E. Cox, R. Austin, J. Sturm. Continuous Particle Separation Through Deterministic Lateral Displacement. Science, 304, 987-990, 2004.

18 M. Hughes. Strategies for Dielectrophoretic Separation in Laboratory-on-a-Chip Systems. Electrophoresis, 23, 2569-2582, 2002.

19 R. Applegate, J. Squier, T. Vestad, J. Oakey, D. Marr, P. Babo, M. Dugan, A. Said. Microfluidic Sorting System Based on Optical Waveguide Integration and Diode Laser Bar Trapping. Lab Chip, 6, 422-426, 2006.

${ }^{20}$ M. Takayasu, D. Kolland, J. Minervini. Continuous Magnetic Separation of Blood Components from Whole Blood. IEEE Transactions on Applied Superconductivity, 10, 927-930.

${ }^{21}$ P. Sethu, M. Anahtar, L. Moldawer, R. Tompkins, M. Toner. Continuous Flow Microfluidic Device for Rapid Erythrocyte Lysis. Analtical Chemistry, 76, 6247-6253, 2004.

${ }^{22}$ V. Hessel, H. Lowe, F. Schonfeld. Review on Patents in Microreactor and Micro Process Engineering. Chemical Engineering Science, 60, 2479-2501, 2005.

23 K. Oh, C. Ahn. A review of microvalves, Journal of Micromechanics and Microengineering. 16(5), 13-39, 2006.

${ }^{24}$ S. Shoji, M. Esashi. Microflow Devices and Systems. Journal of Micromechanics and Microengineering, 4, 157-171, 1994.

25 M. Kersaudy-Kerhoas, R. Dhariwal, M. Desmulliez, L. Jouvet. Hydrodynamic Blood Plasma Separation. Microfluid Nanofluid, 8, 105-114, 2010.

${ }^{26}$ M. Abkarian, A. Viallat. Vesicles and Red Blood Cells in Shear Flow. Soft Matter, 4, 653-657, 2008.

27 M. Abkarian, M. Faivre, A. Viallat. Swinging of Red Blood Cells under Shear Flow. Physics Review Letter, 98, 18-22, 2007. 
${ }^{28}$ S. Yang, A. Undar, J.D. Zahn. A Microfluidic Device for Continuous, Real Time Blood Plasma Separation. Lab Chip, 6, 871-880, 2006.

${ }^{29}$ C. Blattert, R. Jurischka, I. Tahhan, A. Schoth, P. Kerth, W. Menz. Microfluidic Blood Plasma Separation Unit Based on Microchannel Bend Structures. Proceedings of the $3^{\text {rd }}$ Annual International IEEE EMBS Special Topic, 2005.

${ }^{30}$ C. Blattert, R. Jurischka, I. Tahhan, A. Schoth, P. Kerth, W. Menz. Separation of Blood in Microchannel Bends. Proceedings of the $26^{\text {th }}$ Annual International Conference of the IEEE EMBS, 2004.

31 X. Xue, M. K. Patel, M. Kersaudy-Kerhoas, C. Bailey, M. P. Y. Desmulliez, D. Topham. Effect of Fluid Dynamics and Device Mechanism on Biofluid Behaviour in Microchannel Systems: Modelling Biofluids in a Microchannel Biochip Separator. 2009 International Conference on Electronic Packaging Technology \& High Density Packaging (ICEPT-HDP) IEEE, 2009.

32 R. Fahraeus, T. Lindqvist. The Viscosity of the Blood in Narrow Capillary Tubes. 1930.

${ }^{33}$ C. Huang, P. Li, C. Pai, T. Len, C. Jen. Design and Simulation of a Microfluidic Blood-Plasma Separation Chip Using Microchannel Structures. Separation Science and Technology, 45, 42-49, 2010.

34 B. Jo, L. Lerberghe, K. Motsegood, D. Beebe. Three-Dimensional Micro-Channel Fabrication in Polydimethylsiloxane (PDMS) Elastomer. Journal of Microelectromechanical Systems, 9, 76-81, 2000.

35 COMSOL Company. <http://www.comsol.com/products/multiphysics/>

${ }^{36}$ X. Xue, M. Patel, M. Kersaudy-Kerhoas, C. Bailey, M. Desmulliez, D. Topham. Effect of Fluid Dynamics and Device Mechanism on Biofluid Behaviour in Microchannel Systems: Modelling Biofluids in a Microchannel Biochip Separator. 2009 International Conference on Electronic Packaging Technology \& High Density Packaging (ICEPT-HDP) IEEE, 2009.

37 S. Prasad, Y. Yadav, M. Bothara, S. Muthukumar, V. Kunduru. Microfluidic Devices in Nanotechnology. New Jersey: John Wiley \& Sons, Inc., 2010.

38 J. Jong, R. Lammertink, M. Wessling. Membranes and Microfluidics: A Review. Lab chip, 6, 1125-1139, 2006.

39 J. Cooper, J. Chen, Y. Li, C. Lee. Membrane-Based Nanoscale Proteolytic Reactor Enabling Protein Digestion, Peptide Separation, and Protein Identification Using Mass Spectrometry. Analytical Chemistry, 75, 1067-1074, 2003.

40 P. Wang, J. Gao, C. Lee. High-resolution Chiral Separation Using Microfluidics-based Membrane Chromatography. Journal of Chromatography, 942, 115-122, 2001. 
41 X. Wang, C. Saridara, S. Mitra. Microfluidic Supported Liquid Membrane Extraction. Analytical Chimica Acta, 543, 92-98, 2005.

42 B. Chueh, S. Takayama. Leakage-Free Bonding of Porous Membranes into Layered Microfluidic Array Systems. Analytical Chemistry, 79, 3504, 2007.

43 J. Jong, B. ankone, R. Lammertink, M. Wessling. New Replication Technique for the Fabrication of Thin Polymeric Microfluidic Devices with Tunable Porosity. Lab Chip, 5, 1240-1247, 2005.

44 T. Kuo, D. Cannon, Y. Chen, J. Tulock, M. Shannon, J. Sweedler, P. Bohn. Gateable Nanofluidic Interconnects for Multilayered Microfluidic Separation Systems. Analytical Chemistry, 75, 1861-1867, 2003.

45 Pall Life Sciences Corporation. Vivid Plasma Separation Membrane Date Sheet. <http://www.pall.com/pdfs/OEM-Materials-and-Devices/09.2730_VividPlasma_DS 6pg.pdf $>$

${ }^{46}$ H. Darcy. Les Fontaines Publiques de la Ville de Dijon. Dalmont, Paris.

47 Houghton Mifflin Company. Capillary Action. <http://www.thefreedictionary.com/Capillary+force>

${ }^{48}$ E. Oosterbroek, A. Berg. Lab-on-a-chip: Miniaturized Systems for (Bio)Chemical analysis and synthesis. Elsevier Science, second edition.

49 C. Chin, V. Linder, S. Sia. Lab-on-a-Chip Devices for Global Health: Past Studies and Future Opportunities. Lab Chip, 7, 41-57, 2007.

50 D. Figeys, D. Pinto. Lab-on-a-Chip: A Revolution in Biological and Medical Sciences. Analytical Chemistry, 330-335, 2000.

51 I. Graz, S. Lacour. Complementary Organic Thin Film Transistor Circuits 4Fabricated Directly on Silicone Substrates. Organic Electronics, 11, 1815-1820, 2010. 